\title{
Spherical inhomogeneous solutions of Einstein and scalar-tensor gravity: a map of the land
}

\author{
Valerio Faraoni $^{\mathrm{a}, *}$, Andrea Giusti ${ }^{\mathrm{a}, \mathrm{b}}$, Bardia H. Fahim ${ }^{\mathrm{a}}$ \\ ${ }^{a}$ Department of Physics 8 Astronomy, Bishop's University, 2600 College St., \\ Sherbrooke, Québec, Canada J1M 1Z7 \\ ${ }^{b}$ Institute for Theoretical Physics, ETH Zurich, Wolfgang-Pauli-Strasse 27, 8093, \\ Zurich, Switzerland
}

\begin{abstract}
We review spherical and inhomogeneous analytic solutions of the field equations of Einstein and of scalar-tensor gravity, including Brans-Dicke theory, non-minimally (possibly conformally) coupled scalar fields, Horndeski, and beyond Horndeski/DHOST gravity. The zoo includes both static and dynamic solutions, asymptotically flat, and asymptotically Friedmann-LemaîtreRobertson-Walker ones. We minimize overlap with existing books and reviews and we place emphasis on scalar field spacetimes and on geometries that are "general" within certain classes. Relations between various solutions, which have largely emerged during the last decade, are pointed out.
\end{abstract}

Keywords: Exact solution, spherical symmetry, variable order, general relativity, scalar-tensor gravity

\footnotetext{
${ }^{*}$ Corresponding author

Email address: vfaraoni@ubishops.ca (Valerio Faraoni)
} 


\section{Contents}

1 Introduction 4

I Spherical solutions of general relativity 9

2 Vacuum: the Jebsen-Birkhoff theorem and its generalization $\begin{array}{ll}\text { to } \Lambda \neq 0 & 10\end{array}$

2.1 Uniqueness of the KSdS and SAdS metrics . . . . . . . . . . 12

2.2 Simultaneous baldness and cosmic baldness . . . . . . . . . 14

3 No-hair theorems $\quad 15$

4 Spherical scalar field solutions $\quad 17$

4.1 Static case: FJNWBW geometry . . . . . . . . . . . . . . 18

4.2 Solutions with $V(\phi) \neq 0 \ldots \ldots \ldots \ldots . \ldots \ldots 22$

4.3 Time-dependent solutions . . . . . . . . . . . . . . . 23

4.3.1 Wyman's "other" solutions ............ . 24

4.3.2 Husain-Martinez-Nuñez solution . . . . . . . . . 25

4.3 .3 Fonarev spacetime . . . . . . . . . . . . 27

4.3.4 Roberts solution and its generalizations . . . . . . . 30

5 Static Kiselev solution 33

6 Fluid spheres $\quad 36$

6.1 Static fluid spheres in GR . . . . . . . . . . . . 36

6.2 Dynamical fluid spheres in GR . . . . . . . . . . . 38

7 Inhomogeneities embedded in a FLRW universe with fluid 39

7.1 McVittie solutions . . . . . . . . . . . . . . . . 39

7.1.1 Charged and generalized McVittie geometries . . . . 43

7.2 Einstein-Straus, Lemaître-Tolman-Bondi, and Vaidya solutions 43

7.3 Solutions generated by conformal transformations . . . . . . . 44

7.3.1 Non-rotating Thakurta spacetime . . . . . . . . . . 44

7.3.2 Sultana-Dyer spacetime . . . . . . . . . . . . . 46

7.4 Other solutions . . . . . . . . . . . . . . . . . 48

II Spherical solutions of scalar-tensor gravities 48 
8 Scalar-tensor gravity $\quad 49$

8.1 Black holes in scalar-tensor gravity: no-hair theorems . . . . 51

9 The general non-black hole, spherical, static, asymptotically flat solution $\quad 54$

10 A selection of dynamical solutions of Brans-Dicke theory 58

10.1 Clifton-Mota-Barrow spacetimes . . . . . . . . . 59

10.2 Conformally transformed Husain-Martinez-Nuñez spacetime . 61

10.3 Geometry conformal to Fonarev . . . . . . . . . . . . . 61

11 Nonminimally coupled scalar fields $\quad 62$

11.1 Conformal coupling . . . . . . . . . . . . . . 66

11.1.1 BBMB solution . . . . . . . . . . . . . 66

11.1.2 Abreu et al. generalization to scalar field potential . . 67

11.2 Other time-dependent solutions . . . . . . . . . . . . . 68

$\begin{array}{ll}12 f(\mathcal{R}) \text { gravity } & 68\end{array}$

12.1 Black holes and no-hair theorems in $\boldsymbol{f}(\boldsymbol{R})$ gravity . . . . . . 69

12.2 Clifton-Barrow static solution of $\boldsymbol{f}(\boldsymbol{\mathcal { R }})=\boldsymbol{\mathcal { R }}^{\mathbf{1 + \boldsymbol { \delta }}}$ gravity . . . 70

12.3 Clifton's inhomogeneous cosmology in $\mathcal{R}^{1+\delta}$ gravity . . . . . 71

12.4 Conformal image of the Fonarev solution . . . . . . . . . . 71

12.5 Other solutions . . . . . . . . . . . . . . . 72

13 Horndeski gravity $\quad 73$

13.1 Hair or no-hair? . . . . . . . . . . . . . . . . . . 76

13.2 Perturbative and numerical solutions . . . . . . . . . 78

13.3 Babichev-Charmousis class of solutions . . . . . . . . . . 79

14 Beyond Horndeski: DHOST gravity $\quad 82$

$\begin{array}{lr}15 \text { Summary and conclusions } & 87\end{array}$ 


\section{Introduction}

We have now known general relativity (GR) and its Schwarzschild solution describing a spherical isolated object for over a century, during which GR has been put to the test and has found astrophysical applications that were unconceivable at its beginnings. In the last few decades, other analytic solutions of Einstein theory have been discovered and the theory itself has been challenged (questioning GR, however, started early on with Weyl [1] and Eddington [2]). Attempts to quantize gravity and one-loop renormalization necessarily lead to deviations from the Einstein equations and to the introduction of extra degrees of freedom, extra fields, or higher order curvature terms $[3,4,5]$. Historically, the first model of inflation in the early universe, the Starobinsky model [6], incorporated some of these quadratic corrections by promoting the Einstein-Hilbert Lagrangian $\mathcal{R}$ (where $\mathcal{R}$ is the Ricci scalar) to $\mathcal{R}+\alpha \mathcal{R}^{2}$. It is now known that this correction is equivalent to introducing a massive scalar field in addition to gravity. So, at least the Starobinsky inflationary scenario includes corrections to the Einstein-Hilbert action [6].

The surprising discovery, made with Type Ia supernovae $[7,8,9,10$, $11,12]$ that the present expansion of the universe is accelerated has led cosmologists to postulate a completely ad hoc dark energy, which is possibly a cosmological constant $\Lambda$ suffering from the notorious cosmological constant problems $[13,14]$. Quintessence models of dark energy $([15,16,17,18,19$, 20, 21, 22], see [23] for a review) are not well-motivated theoretically. As a consequence, although fitting most data, the standard model of cosmology, the $\Lambda$-Cold Dark Matter $(\Lambda \mathrm{CDM})$ model, appears unnatural. In fact, if the cosmic acceleration is attributed to a cosmological constant, it suffers from the fine-tuning and coincidence problems [23]. If $\Lambda \mathrm{CDM}$ is taken to mean cold dark matter plus dark energy, it remains problematic because dark energy was introduced ad hoc in the theoretical literature to explain the cosmic acceleration and its nature is completely unknown, as testified by the plethora of vastly different dark energy models in the literature. In this sense, its introduction seems unnatural. In any case, the presence of a speculative and exotic dark energy of completely unknown nature remains deeply unsatisfactory.

Starting in 2003 [24, 25], this situation has driven many authors to explain the present cosmic acceleration by modifying Einstein gravity at large scales instead of invoking an ad hoc dark energy. This is the most pressing need to explore modified theories of gravity, among which the so-called $f(\mathcal{R})$ theories $([24,25]$, see $[26,27,28]$ for reviews) are very popular. This 
fact has caused a resurgence of interest in modified gravity and in particular in scalar-tensor gravity, a broader category known since at least 1961 [29] in which $f(\mathcal{R})$ theories fall as a subfamily. The discovery of gravitational waves with the $L I G O$ and $V I R G O$ laser interferometric detectors [30, 31, 32] in 2015 and the 2019 Event Horizon Telescope imaging of the near-horizon region of the M87 black hole [33, 34, 35, 36, 37, 38] have provided unprecedented tools and motivation to study the detailed physics near black holes and compact objects in both GR and alternative gravity. The comparison of observations and theoretical predictions requires the knowledge of analytical solutions in these theories and of the phenomenology of test particles (massive and massless) and fluids in these geometries. Without these background geometries it is impossible to compute strong field effects and these solutions must necessarily be stable ones. What is more, due to the unexpectedly high black hole masses measured in the LIGO and VIRGO events, primordial black holes have attracted again attention [39, 40]. Their importance is that, if they abound in the universe, they can be significant dark matter components. This motivates the study of toy models (i.e., analytic solutions) in which such objects, if they exist, evolve on time scales not completely negligible with respect to the Hubble scale (e.g., $[41,42])$.

In summary, this renewed activity in cosmology and astrophysics has led to the need of a better understanding of the physics of black holes and other objects of astrophysical or mathematical interest in these theories, and to deeper studies of their analytic solutions. The search for analytic solutions of a physical theory unveils its physical aspects and promotes physical insight. Exact solutions of the field equations of non-linear theories are difficult to find, and they are usually found only by simplifying the problem which, in practice, means by assuming symmetries. Free from as many complications as possible, it is easier to gain physical insight by examining symmetric solutions which allow one to focus on a few aspects of the theory at a time. Certainly this has been the case for the Schwarzschild solution of GR, which has led to the discovery of black holes and to the investigation of many astrophysical and fundamental physics phenomena and, indirectly (through black hole mergers), to the discovery of gravitational waves. When trying to make sense of a theory of gravity, one of the first aspects to examine consists of the available exact solutions of its field equations and, among these, its spherically symmetric solutions describing black holes, stars, and possibly other objects. It is unavoidable to compare these solutions with the corresponding one of GR, i.e., the Schwarzschild geometry [43].

Historically, there have been attempts to find exact spherically symmetric solutions of the field equations of theories of gravity describing increas- 
ingly complicated physical situations: vacuum, vacuum with a cosmological constant $\Lambda$, electrovacuum, perfect fluids, imperfect fluids, scalar fields in the context of GR, and then situations in which the scalar field has gravitational nature and is an unavoidable ingredient of the theory of gravity, as in scalar-tensor theories. In practice, solving the field equations directly is challenging and many attempts to generate new analytic solutions have relied on the knowledge of previously existing solutions in different physical contexts. These studies have unveiled relations between different geometries and between different theories (for example, solution-generating techniques have employed conformal transformations relating GR with scalar-tensor gravity through the use of different conformal frames).

Here we review analytic solutions of GR (part I) and of scalar-tensor gravity (part II) which are spherically symmetric, asymptotically or nonasymptotically flat, static or dynamical. We will not repeat the discussion of the most well-known solutions that are found in standard books and reviews (especially those sourced by a perfect or an imperfect fluid), nor will we discuss at length the mathematical structure of the solutions, their algebraic classification, conformal diagrams, or their causal structure. For these aspects, we refer the reader to the monumental work [44] on analytic solutions of the Einstein equations, and to [45, 46, 47, 48, 49] for other aspects, more detailed discussions, and recent developments. Here, instead, we focus on exact solutions within certain classes, pointing out the relations between various families of exact solutions that have emerged during the last few decades.

Of course, one can write down a metric tensor and run the Einstein equations from left to right à la Synge, computing an effective energy-momentum tensor which, in general, violates all the energy conditions [50] and is completely unphysical. We do not consider such solutions unless the effective energy momentum tensor that sources them is physically reasonable.

Solutions containing a scalar field occupy a prominent position in this review. This scalar could be a matter field in Einstein theory (as would be, for example, the recently discovered Higgs boson of the Standard Model of particle physics $[51,52])$. Alternatively, the scalar field could be a gravitational field in an alternative theory of gravity, such as the Brans-Dicke or the dilaton fields. Among the wide spectrum of alternative theories of gravity, we restrict ourselves to Brans-Dicke gravity [29], its scalar-tensor generalizations [53, 54, 55] including the wide class of $f(\mathcal{R})$ theories $[26,27,28]$, Horndeski gravity [56], and degenerate higher order scalar-tensor theories $[57,58]$. We exclude torsion and, in order to keep the material contained, we restrict to spherically symmetric solutions of the field equations and to 
four spacetime dimensions (for higher-dimensional solutions, see [59,60] and the references therein).

Certain dynamical solutions in this review are believed to represent black holes. In the dynamical case the black hole boundary, and the black hole concept itself, are not defined by the usual notion of event horizon, which is a null surface and a causal boundary (i.e., a connected component of the boundary of the causal past of future null infinity [50]). The teleological notion of event horizon requires the knowledge of the entire causal structure of spacetime and is impractical in dynamical situations and in numerical relativity. Instead, dynamical black holes are defined quasilocally by apparent horizons and marginally trapped surfaces. A drawback of this definition (see $[61,62,47,48]$ for reviews) is that apparent horizons depend on the choice of the spacetime foliation, as exemplified dramatically by the fact that the Schwarzschild spacetime admits non-symmetric foliations without apparent horizons $[63,64]$. However, in the presence of spherical symmetry (to which we restrict here), all spherically symmetric foliations produce the same apparent horizons [65].

We follow the notation of Ref. [50]. The metric signature is $(-+++)$, we use geometric units in which the speed of light $c$ and Newton's constant $G$ assume the values $c=G=1, \kappa \equiv 8 \pi G$, and $\square \equiv g^{a b} \nabla_{a} \nabla_{b}$ is d'Alembert's operator. The Riemann and Ricci tensors are given in terms of the Christoffel symbols $\Gamma_{a b}^{d}$ by

$$
\begin{gathered}
\mathcal{R}_{a b c}{ }^{d}=\Gamma_{a c, b}^{d}-\Gamma_{b c, a}^{d}+\Gamma_{a c}^{e} \Gamma_{e b}^{d}-\Gamma_{b c}^{e} \Gamma_{e a}^{d}, \\
\mathcal{R}_{a c} \equiv \mathcal{R}_{a b c}{ }^{b}=\Gamma_{a c, b}^{b}-\Gamma_{b c, a}^{b}+\Gamma_{a c}^{d} \Gamma_{d e}^{e}-\Gamma_{b c}^{d} \Gamma_{d a}^{b},
\end{gathered}
$$

and $\mathcal{R} \equiv g^{a b} \mathcal{R}_{a b}$ is the Ricci curvature.

Let us define some terminology that will be used in this work. A perfect fluid is identified by the energy-momentum tensor

$$
T_{a b}=(P+\rho) u_{a} u_{b}+P g_{a b},
$$

where $u^{c}$ is the fluid four-velocity (normalized to $u^{c} u_{c}=-1$ ), $\rho$ is its energy density, and $P$ is the isotropic pressure. In practice, $P$ and $\rho$ are not unrelated and there will be an equation of state relating them but, technically, this is not required by the definition above. This fact opens the possibility that, when looking for formal analytic solutions of the field equations sourced by a fluid, one can adjust $P$ and $\rho$, which depend on the spacetime point, so that a certain geometry can be engineered to satisfy the field equations. This procedure detracts from the physics, in the sense that it 
would be much preferable to assign a priori an equation of state valid everywhere, for example a barotropic equation of state of the form $P=w \rho$ with $w=$ constant, and then solve the field equations. In practice, this method is not always followed or does not produce new solutions. The less than satisfactory procedure of determining the equation of state a posteriori is not unique to spherical solutions (it is used, for example, already in cosmology where the field equations are simpler). There are instances where this procedure does not cause harm, for example when one decides to regard a minimally coupled scalar field as an effective perfect fluid. In this case, the equation of state $P_{\text {eff }}=w \rho_{\text {eff }}$ exhibits a dynamical equation of state parameter $w=w\left(x^{\alpha}\right)$ depending on the spacetime position. Other times, the equation of state of the fluid does not originate in a field theory but is derived in order to satisfy the equations and, while it may reduce to a satisfactory equation of state in certain regions of spacetime, it will remain rather ad hoc in other regions. This is the case, for example, for the McVittie solution of the Einstein equations describing a central object embedded in a cosmological space, that will be discussed in Sec. 7. But then it turns out that the McVittie solution is a also a solution of a different theory of gravity that does not require an ad hoc fluid equation of state. This new perspective somehow rescues the McVittie geometry from the limbo of solutions with questionable motivation (the borders of which are rather subjective). The possibility of having different sources for a certain GR geometry, or that the latter solves a different theory of gravity, remains unexplored for most of the solutions reported in this review.

Another term that is widely used in this work should be defined. We refer to vacuum to denote the absence of a proper matter stress-energy tensor. In the Einstein equations

$$
\mathcal{R}_{a b}-\frac{1}{2} g_{a b} \mathcal{R}+\Lambda g_{a b}=8 \pi T_{a b}^{(\mathrm{m})},
$$

the term containing the cosmological constant $\Lambda$ can be taken to the right hand side and regarded as an effective stress-energy tensor $T_{a b}^{(\Lambda)}=-\frac{\Lambda}{8 \pi} g_{a b}$ : we still refer to this situation as "vacuum with a cosmological constant". Similarly, in scalar-tensor gravity, one can write the field equations in the form of effective Einstein equations

$$
\mathcal{R}_{a b}-\frac{1}{2} g_{a b} \mathcal{R}=8 \pi T_{a b}^{(\mathrm{m})}+8 \pi T_{a b}^{(\mathrm{eff})},
$$

where $T_{a b}^{(\mathrm{m})}$ describes a "real" matter source while $T_{a b}^{(\mathrm{eff})}$ contains all the terms describing extra degrees of freedom or higher curvature terms which 
form an effective (as opposed to "real") stress-energy tensor. In this context, "vacuum scalar-tensor gravity" denotes again the situation $T_{a b}^{(\mathrm{m})}=0$ even though $T_{a b}^{(\mathrm{eff})} \neq 0$.

\section{Part I}

\section{Spherical solutions of general relativity}

We begin by reviewing spherical solutions of the Einstein equations. Proceeding in order of increasing complexity, one is naturally led to consider vacuum, vacuum with a cosmological constant, and then simple forms of matter: electrovacuum, a scalar field, a perfect, and then an imperfect fluid.

Without loss of generality, the spherically symmetric line element in four spacetime dimensions can be written in polar coordinates $(t, r, \vartheta, \varphi)$ as (see Sec. 11.7 of Ref. [66])

$$
d s^{2}=-A^{2}(t, R) d t^{2}+B^{2}(t, R) d R^{2}+R^{2} d \Omega_{(2)}^{2},
$$

using the areal radius $R$ as the radial coordinate, and where $d \Omega_{(2)}^{2} \equiv d \vartheta^{2}+$ $\sin ^{2} \vartheta d \varphi^{2}$ is the line element on the unit 2-sphere.

The situation $A^{2} B^{2}=1$ is of special interest $[67,68,69]$ and highlights special algebraic properties of the geometry. Jacobson has shown [69] (also in higher dimension) that it is equivalent to require that the double projection of the Ricci tensor $\mathcal{R}_{a b} \ell^{a} \ell^{b}$ onto radial null vectors $\ell^{a}$ vanishes. Another characterization is that the restriction of the Ricci tensor to the $(t, R)$ subspace be proportional to the restriction of the metric $g_{a b}$ to the same subspace [69]. A third characterization is that the areal radius $R$ is an affine parameter along radial null geodesics [69]. GR geometries satisfying these conditions are varied, ranging from vacuum, electrovacuum with either Maxwell or non-linear Born-Infeld electrodynamics, to the "string hedgehog" global monopole [70].

A convenient form of the gauge (6) is $[71,72]$

$$
\begin{aligned}
d s^{2}= & -\mathrm{e}^{-\Phi(t, R)}\left(1-\frac{2 M_{\mathrm{MSH}}(t, R)}{R}\right) d t^{2} \\
& +\frac{d R^{2}}{1-2 M_{\mathrm{MSH}}(t, R) / R}+R^{2} d \Omega_{(2)}^{2},
\end{aligned}
$$


where $M_{\mathrm{MSH}}(R)$ is the Misner-Sharp-Hernandez mass $[73,74]$ enclosed in a sphere of radius $R$.

In view of astrophysical applications, the motion of massive particles and fluids around a black hole is of great interest. Since these motions can be quite complicated, pseudo-Newtonian potentials have been introduced that give an effective simplified description of timelike geodesics around Schwarzschild and Kerr black holes [75, 76, 77, 78, 79, 80, 81, 82, 83, 84, 85, 86, 87, 88]. The Paczynski-Wiita pseudopotential for the Schwarzschild black hole (11) is simply [75, 77]

$$
\phi_{\mathrm{PW}}(R)=-\frac{m}{R-2 m} .
$$

This potential reproduces exactly the radius of the innermost stable circular orbit ("ISCO" or "marginally stable orbit"), that of the marginally bound orbit, and the form of the Keplerian angular momentum $L(R)$. It reproduces, accurately but not exactly, the Keplerian angular velocity and the radial epicyclic frequency $[75,77]$. Therefore, the structure of the phase space of timelike geodesics in the Schwarzschild metric does not differ much from the one obtained with the pseudopotential [89].

The motion of massive test particles and fluids around black holes offers potential tests of the theory of gravity using observations of these motions near a black hole horizon. A pseudopotential can be introduced for any static and spherically symmetric metric of the form (6) with $A=A(R)$ and $B=B(R)$, obtaining

$$
\phi(R)=\frac{1}{2}\left[1-\frac{1}{A^{2}(R)}\right]
$$

in the approximation of particle velocities $v \ll 1$ [77]. If the further as-

sumption $A^{2}(R) B^{2}(R)=1$ holds, then this pseudopotential can be written as [90]

$$
\phi(R)=-\frac{M_{\mathrm{MSH}}(R)}{R-2 M_{\mathrm{MSH}}(R)},
$$

as follows immediately from the gauge (7) with $\Phi=0$.

\section{Vacuum: the Jebsen-Birkhoff theorem and its generalization to $\Lambda \neq 0$}

As is well-known from any GR textbook, the Jebsen-Birkhoff theorem [91, 92] establishes that the unique vacuum, spherically symmetric, and 
asymptotically flat solution of the Einstein equations is the Schwarzschild geometry $[43]^{1}$

$$
d s^{2}=-\left(1-\frac{2 m}{R}\right) d t^{2}+\frac{d R^{2}}{1-2 m / R}+R^{2} d \Omega_{(2)}^{2},
$$

and the generalization of the theorem to electrovacuum establishes the uniqueness of the Reissner-Nordström solution under the same assumptions (see [96] for a review of GR black holes). A weak form of the Jebsen-Birkhoff theorem states that the solution is static for special forms of the matter energy-momentum tensor $[97,98,99,100]$. In the literature, one finds also almost-Birkhoff theorems that study small deviations from spherical symmetry or from vacuum $[101,102,103,104]$.

The Jebsen-Birkhoff theorem can be extended to vacuum with a cosmological constant $\Lambda$ in a straightforward way concluding that, in this case, the unique spherical solution of the vacuum Einstein equations is the Kottler/Schwarzschild-de Sitter (KSdS) geometry [105] if $\Lambda>0$ and the asymptotics are de Sitter, or is the Schwarzschild-anti-de Sitter (SAdS) geometry if, instead, $\Lambda<0$ and the asymptotics are anti-de Sitter. In the following, we refer explicitly to the KSdS case, but the discussion for $\Lambda<0$ and SAdS is straightforward. The standard textbook discussion of the Jebsen-Birkhoff theorem is obtained as a special case by setting $\Lambda=0$.

In locally static (Schwarzschild-like or "curvature") coordinates $(T, R, \vartheta, \varphi)$, the KSdS metric reads

$$
d s^{2}=-\left(1-\frac{2 m}{R}-H^{2} R^{2}\right) d T^{2}+\frac{d R^{2}}{1-\frac{2 m}{R}-H^{2} R^{2}}+R^{2} d \Omega_{(2)}^{2},
$$

where $H=\sqrt{\Lambda / 3}$ and $m$ are positive constants.

Surprisingly, modern relativity textbooks do not make reference to the Jebsen-Birkhoff theorem when $\Lambda \neq 0$, and the literature is ambiguous to this regard. Occasionally, there are explicit statements of the uniqueness of the $\mathrm{S}(\mathrm{A}) \mathrm{dS}$ space (e.g., $[106,104,107])$ and a proof of the extension of the theorem to $\Lambda \neq 0$ was given in Synge's 1960 textbook [108] (without mention of Kottler's 1918 paper [105]). A simple proof in null coordinates was given by Schleich and Witt [109] and there are mathematically more sophisticated proofs $[110,111,112,113]$. As for the Schwarzschild solution, uniqueness

\footnotetext{
${ }^{1}$ In spacetime dimension $d>4$, the uniqueness of the corresponding "SchwarzschildTangherlini" solution was proved by Bronnikov and Melnikov [93], and by Gibbons et al. $[94,95]$.
} 
is associated with stability of the KSdS and SAdS solutions with respect to perturbations, as established in [114, 115, 116, 117]. However, the evidence of the uniqueness of KSdS and SAdS is downplayed in the literature and papers exist that erroneusly state the existence of spherical solutions of the vacuum Einstein equations with $\Lambda>0$ alternative to $\operatorname{KSdS}[118,119,120]$, which would contradict uniqueness. The problem is that the line element is written in rather complicated coordinates and is not recognized as the KSdS line element, or an incorrect coordinate transformation leads to a line element formally different from the KSdS one. Furthermore, there are solutions of the Einstein equations that represent central inhomogeneities embedded in Friedmann-Lemaître-Robertson-Walker (FLRW) spaces and that apparently reduce to alternatives to the KSdS solution in the special case when the FLRW "background" ${ }^{2}$ reduces to de Sitter [121, 122, 123, 124]. However, these are solutions with matter sources and not $\Lambda$-vacuum solutions. These putative alternatives have been shown to be either KSdS disguised by unconventional coordinates, or genuinely different solutions in the presence of matter [125].

\subsection{Uniqueness of the KSdS and SAdS metrics}

Here we prove the uniqueness of the KSdS and SAdS spacetimes using a particular gauge but the result is, of course, gauge-independent. The vacuum Einstein equations

$$
G_{a b}=-\Lambda g_{a b}
$$

yield, in the gauge (6),

$$
\begin{aligned}
& \frac{\dot{B}}{R B}=0, \\
& \frac{2 B^{\prime}}{B^{3} R}-\frac{1}{B^{2} R^{2}}+\frac{1}{R^{2}}=\Lambda, \\
& \frac{2 A^{\prime}}{A R}-\frac{B^{2}}{R^{2}}+\frac{1}{R^{2}}=-\Lambda B^{2}, \\
& \frac{A^{\prime} B}{A}-B^{\prime}-\frac{R B^{2} \ddot{B}}{A^{2}}+\frac{R \dot{A} \dot{B} B^{2}}{A^{3}}-\frac{R A^{\prime} B^{\prime}}{A}+\frac{R A^{\prime \prime} B}{A}=-\Lambda R B^{3},
\end{aligned}
$$

\footnotetext{
${ }^{2}$ We use quotation marks because, due to the non-linearity of the field equations, it is impossible to split a metric into a background plus a deviation from it (with the exception of Kerr-Schild metrics [44]).
} 
where an overdot and a prime denote differentiation with respect to $t$ and $R$, respectively. ${ }^{3}$ Equation (14) implies that $B=B(R)$ and, setting $\dot{B}$ and $\ddot{B}$ to zero in Eq. (17) as a consequence, Eq. (15) yields

$$
\left(\frac{R}{B^{2}}\right)^{\prime}=1-\Lambda R^{2}
$$

with solution

$$
B^{2}(R)=\frac{1}{1+\frac{C}{R}-\frac{\Lambda R^{2}}{3}},
$$

with $C$ is an integration constant. In the limit $\Lambda \rightarrow 0$, one must recover the Schwarzschild line element (11) for a mass $m$, which fixes the integration constant to $C=-2 m$ and

$$
B^{2}=\frac{1}{1-\frac{2 m}{R}-\frac{\Lambda R^{2}}{3}} .
$$

Now Eq. (16) yields

$$
\left(\ln A^{2}\right)^{\prime}=\left[\ln \left(1-\frac{2 m}{R}-\frac{\Lambda R^{2}}{3}\right)\right]^{\prime}
$$

with general solution

$$
A^{2}(R)=\mathrm{e}^{D(t)}\left(1-\frac{2 m}{R}-\frac{\Lambda R^{2}}{3}\right)
$$

where $D(t)$ is an integration function of time. By rescaling the time coordinate so that $d T=\mathrm{e}^{D(t) / 2} d t$, the line element necessarily becomes

$$
d s^{2}=-\left(1-\frac{2 m}{R}-\frac{\Lambda R^{2}}{3}\right) d T^{2}+\frac{d R^{2}}{1-\frac{2 m}{R}-\frac{\Lambda R^{2}}{3}}+R^{2} d \Omega_{(2)}^{2},
$$

which is the KSdS geometry if $\Lambda>0$ (and then $H=\sqrt{\Lambda / 3}$ ), or the SAdS one if $\Lambda<0$. Therefore, KSdS [SAdS] is the unique vacuum spherical solution if $\Lambda>0$ [if $\Lambda<0$ ]. It reduces to the Schwarzschild geometry if $\Lambda=0$.

\footnotetext{
${ }^{3}$ The $\vartheta-\vartheta$ and $\varphi-\varphi$ components of the Einstein equations provide the same information because of the symmetry.
} 


\subsection{Simultaneous baldness and cosmic baldness}

Well-known cosmic no-hair theorems state that, with a few exceptions (i.e., overdense Bianchi models that collapse before the cosmological constant has a chance to dominate the cosmic dynamics), de Sitter space behaves as an attractor in the late-time dynamics of the universe $[126,127$, 128]. In an analogy, no-hair theorems establish the uniqueness of the Schwarzschild solution among GR black holes and exclude the possibility of fields living in their exterior, since they would make the geometry deviate from Schwarzschild [129, 130, 131, 132, 133, 134, 135, 136, 137, 138, 139, 140, 141]. Moreover, the KSdS solution is unique, as shown above, and is perturbatively stable $[114,115,116,117]$. Since the KSdS geometry combines black hole and de Sitter physics, it is natural to expect that simultaneous no-hair and cosmic no-hair theorems exist and that the KSdS spacetime acts as a late-time attractor for the dynamics of time-dependent spherical spacetimes containing a central inhomogeneity in the presence of a positive $\Lambda$. This idea has not been explored much, probably due to the difficulty of handling the Einstein equations in the presence of matter. As a starter in this direction, a non-perturbative result in the presence of an imperfect fluid was derived in $[125]$.

Consider the Einstein equations with matter

$$
G_{a b}=-\Lambda g_{a b}+8 \pi T_{a b}
$$

and assume spherical symmetry, then the line element is (6). Further assume that the geometry is asymptotically de Sitter, i.e., that there is a de Sitterlike cosmological horizon (with areal radius $R_{\mathrm{H}}$ ) and the solution of (24) reduces to (12) as $R \rightarrow R_{\mathrm{H}}^{-}$. Then, assume that the energy-momentum tensor in the right hand side of the Einstein equations

$$
\begin{aligned}
& \frac{\dot{B}}{B R}=4 \pi T_{01}, \\
& A^{2}\left(\frac{2 B^{\prime}}{B^{3} R}-\frac{1}{B^{2} R^{2}}+\frac{1}{R^{2}}\right)=\Lambda A^{2}+8 \pi T_{00}, \\
& \frac{2 A^{\prime}}{A R}-\frac{B^{2}}{R^{2}}+\frac{1}{R^{2}}=-\Lambda B^{2}+8 \pi T_{11}, \\
& \frac{A^{\prime} B}{A}-B^{\prime}-\frac{R B^{2} \ddot{B}}{A^{2}}+\frac{R \dot{A} \dot{B} B^{2}}{A^{3}}-\frac{R A^{\prime} B^{\prime}}{A} \\
& +\frac{R A^{\prime \prime} B}{A}=\left(-\Lambda R^{2}+8 \pi T_{22}\right) \frac{B^{3}}{R},
\end{aligned}
$$


describes an imperfect fluid with constant equation of state and a purely spatial radial heat flow with flux density $q^{a}$,

$$
\begin{aligned}
T_{a b} & =(P+\rho) u_{a} u_{b}+P g_{a b}+q_{a} u_{b}+q_{b} u_{a}, \\
P & =w \rho, \quad w=\text { const. } \\
u^{a} u_{a} & =-1, \quad q^{c} q_{c}>0, \quad q^{c} u_{c}=0 .
\end{aligned}
$$

As shown in [125], either the matter fluid reduces to a cosmological constant at late times, in which case the vacuum uniqueness theorem for KSdS holds, or else both $\rho$ and $P=w \rho$ become subdominant and $\Lambda$ comes to dominate the expansion at late times while $\rho$ and $P$ become unimportant - then the solution reduces to KSdS.

\section{No-hair theorems}

No-hair theorems have a long history. Attempts to evade the JebsenBirkhoff theorem include the possibility of a matter source in the black hole exterior, for example a fluid or a scalar field. However, it is not so easy because this matter becomes physically pathological. For example, according to an early no-hair result by Chase [129], a four-dimensional static, massless, asymptotically flat scalar field solution with a simply connected event horizon has the squared norm of the timelike Killing vector diverging there. The only solution is Schwarzschild, corresponding to $\phi \equiv 0$ outside the horizon, or the Fisher-Janis-Newman-Winicour-Buchdahl-Wyman ("FJNWBW") solution containing a naked singularity, which is described below, see Eqs. (42) and (43). The remaining possibility of the scalar field being singular on the horizon is not mentioned explicitly by Chase [129], who views the central naked singularity as a horizon shrunk to a point.

Christodoulou has shown [142] that the naked singularity of the FJNWBW geometry is unstable to gravitational collapse. ${ }^{4}$ In numerical studies of scalar field collapse to a black hole in spherical symmetry, the scalar field either collapses below the Schwarzschild horizon forming a Schwarzschild black hole, or disperses to infinity [144]. Exact scalar field solutions describing other configurations (i.e., naked singularities) exist, but they are

\footnotetext{
${ }^{4}$ See Ref. [143] for a version of Christodolou's theorem with slightly relaxed assumptions.
} 
unstable and do not form in numerical collapse. ${ }^{5}$ They are discussed in the next section.

No-hair theorems for a scalar field with potential $V(\phi)$ that is monotonically increasing and convex have been proved by Bekenstein [133, 131, 132]. A generalization to general positive potentials $V(\phi)$ was given in [147, 148], followed by a version of the no-hair theorem for scalar multiplets [137] (and by a no-hair theorem for nonminimally coupled scalars, which are discussed later $[149,134])$. The no-hair theorems, however, fail in Einstein-Yang-Mills theory $[150,151,152]$, in Einstein-Skyrme theory [153, 154], and in situations where the above scenarios couple with dilaton $[155,156]$ or Higgs $[157,158]$ fields.

From the physical point of view, the parameters charactering stationary black holes are conserved quantities associated with a Gauss law: mass, angular momentum, electric and magnetic charges. Therefore, the Maxwell field satisfying the Gauss law leads to the absence of Maxwell hair and to the uniqueness of the Reissner-Nordström solution. Moreover, according to theorems by Israel [159, 160], staticity implies spherical symmetry for electrovacuum GR, from which the uniqueness of the Reissner-Nordström black hole follows. More in general, Hawking's strong rigidity theorem $[161,162]$ states that a stationary black hole in GR is either spherical or axisymmetric when the null energy condition is satisfied (this theorem underlies the proof of Hawking's original no-hair theorem [163] for Brans-Dicke black holes). The Hawking and Israel theorems, however, do not extend to non-Abelian Yang-Mills fields. In this respect a scalar field is similar: since it has no associated Gauss law, it is in principle possible to have scalar hair.

Non-Abelian gauge theories with gravity typically violate the no-hair theorem, especially when they possess solitons in the limit to Minkowski spacetime. Non-Abelian Yang-Mills fields in GR exhibit a short-ranged gauge field which is not killed by setting to zero the conserved charges. In addition, non-Abelian Yang-Mills black holes in GR can be static but still deviate from spherical symmetry, thus evading the Israel theorems and allowing for hair. Since these fields violate some of the ingredients necessary for the proofs of the Hawking and Israel theorems, which in turn are needed to prove no-hair results, it is not too surprising that they exhibit hair. Skyrme fields exhibit some of these features of non-Abelian YangMills fields and admit hair as well (see [150, 151, 152, 153, 154] for more detailed discussions).

\footnotetext{
${ }^{5}$ See Refs. $[145,146]$ for an overview of gravitational collapse.
} 
Even in scalar-tensor gravity with a simple Brans-Dicke-like field, it becomes more difficult to make statements when the black holes are not asymptotically flat. A no-hair theorem for asymptotically anti-de Sitter black holes has been proved when $\phi$ settles asymptotically in a global negative minimum of the potential $V(\phi)$ [164]. Scalar field potentials not bounded from below, although unphysical in GR and scalar-tensor gravity, can become physical in supergravity and string theories [165] - the reader should bear in mind that, in this review, we restrict ourselves to GR and scalar-tensor gravity.

\section{Spherical scalar field solutions}

The next step in the search for spherically symmetric solutions consists of introducing a relatively simple form of matter: a scalar field minimally coupled to the Ricci curvature and described by the stress-energy tensor

$$
T_{a b}^{(\phi)}=\nabla_{a} \phi \nabla_{b} \phi-\frac{1}{2} g_{a b} \nabla^{c} \phi \nabla_{c} \phi-\frac{V(\phi)}{2} g_{a b},
$$

where $V(\phi)$ is the scalar field potential. The action is

$$
S=\int d^{4} x \sqrt{-g}\left[\frac{\mathcal{R}}{2 \kappa}-\frac{1}{2} \nabla_{a} \phi \nabla^{a} \phi-V(\phi)\right],
$$

and the coupled Einstein-Klein-Gordon equations are

$$
\begin{aligned}
& \mathcal{R}_{a b}-\frac{1}{2} g_{a b} \mathcal{R}=8 \pi T_{a b}^{(\phi)}, \\
& \square \phi-\frac{d V}{d \phi}=0 .
\end{aligned}
$$


Assuming spherical symmetry, staticity, and the line element in the gauge (6), these equations take the form

$$
\begin{aligned}
& \frac{\dot{B}}{B}=4 \pi R \dot{\phi} \phi^{\prime} \\
& \frac{1}{R^{2}}+\frac{2 B^{\prime}}{B^{3} R}-\frac{1}{B^{2} R^{2}}=\frac{8 \pi}{A^{2}}\left[\dot{\phi}^{2}+\frac{A^{2}}{2}\left(-\frac{\dot{\phi}^{2}}{2}+\frac{\phi^{2}}{2}\right)+V A^{2}\right] \\
& \frac{2 A^{\prime}}{A R}-\frac{B^{2}}{R^{2}}+\frac{1}{R^{2}}=8 \pi\left[\phi^{\prime 2}-\frac{B^{2}}{2}\left(-\frac{\dot{\phi}^{2}}{A^{2}}+\frac{\phi^{\prime 2}}{B^{2}}\right)-V B^{2}\right] \\
& \frac{R}{B^{3}}\left(\frac{A^{\prime} B}{A}-B^{\prime}-\frac{R B^{2} \ddot{B}}{A^{2}}+\frac{R \dot{A} \dot{B} B^{2}}{A^{3}}-\frac{R A^{\prime} B^{\prime}}{A^{3}}\right. \\
& \left.+\frac{R A^{\prime \prime} B}{A}\right)=8 \pi\left[-\frac{R^{2}}{2}\left(-\frac{\dot{\phi}^{2}}{A^{2}}+\frac{\phi^{\prime 2}}{B^{2}}\right)-V R^{2}\right]
\end{aligned}
$$

\subsection{Static case: FJNWBW geometry}

Naturally, one first searches for solutions with a free scalar field (i.e., $V(\phi) \equiv 0)$, in which case the field equations simplify to

$$
\begin{aligned}
& \mathcal{R}_{a b}=8 \pi \nabla_{a} \phi \nabla_{b} \phi, \\
& \square \phi=0 .
\end{aligned}
$$

Their general solution which is static, spherically symmetric, and asymptotically flat is the Fisher solution [166], also known as Janis-Newman-WinicourBuchdahl-Wyman solution (herafter referred to as the FJNWBW geometry). This solution was rediscovered several times $[167,168,169,170,171,172$, $173,174]$ by authors who did not recognize it in different coordinate systems, or were unaware of its previous derivation. A simple proof of its uniqueness is given by Wyman in [170]. It does not describe a black hole: it has no horizons and represents a central naked singularity at areal radius $R=0$. Roberts rederived this solution in null coordinates [175] whereas Agnese and La Camera studied it in the Newman-Penrose formalism [173]. The line 
element and scalar field are $[166,170]$

$$
\begin{aligned}
d s^{2}= & -\mathrm{e}^{\alpha / r} d t^{2}+\mathrm{e}^{-\alpha / r}\left(\frac{\gamma / r}{\sinh (\gamma / r)}\right)^{4} d r^{2} \\
& +\mathrm{e}^{-\alpha / r}\left(\frac{\gamma / r}{\sinh (\gamma / r)}\right)^{2} r^{2} d \Omega_{(2)}^{2}, \\
\phi= & \frac{\phi_{*}}{r}, \quad \phi_{*}=\frac{-\sigma}{4 \sqrt{\pi}},
\end{aligned}
$$

where $\alpha, \gamma$, and $\phi_{*}$ are constants, $\sigma$ is a scalar charge, and one can take $\gamma \geq 0$ without loss of generality. These three constants are related by [170]

$$
4 \gamma^{2}=\alpha^{2}+2 \sigma^{2} .
$$

If $\sigma=0$ the constants $\alpha$ and $\gamma$ both vanish, the scalar field vanishes, and the solution (42) reduces to the Minkowski metric. In the notation of Wyman [170] that we follow, the relation (44) between the constants $\alpha, \gamma$ and $\sigma$ does not allow one to see this fact. However, in the original derivation of Eq. (42) in [170], one sees that the vanishing of $\sigma$ implies the vanishing of both $\alpha$ and $\gamma$.

The areal radius is

$$
R(r)=\gamma \frac{\mathrm{e}^{-\frac{\alpha}{2 r}}}{\sinh (\gamma / r)} \rightarrow 0^{+} \text {as } r \rightarrow 0^{+}
$$

and there is a spacetime singularity at $R=0$ (or $r=0$ ) since the Ricci scalar

$$
\mathcal{R}=8 \pi \nabla^{c} \phi \nabla_{c} \phi=\frac{8 \pi \phi_{*}^{2}}{\gamma^{4}} \mathrm{e}^{\alpha / r} \sinh ^{4}(\gamma / r)
$$

diverges as $r \rightarrow 0$. This singularity is naked. In fact, horizons that could cover this singularity correspond to roots of the equation $\nabla^{c} R \nabla_{c} R=0$ (single roots corresponding to black hole horizons and double roots to wormhole throats $[73,71,47])$, and no such root exists. ${ }^{6}$ To see this, one computes

$$
\nabla^{c} R \nabla_{c} R=\frac{1}{16 \gamma^{2}}\left[(\alpha+2 \gamma) \mathrm{e}^{\gamma / r}-(\alpha-2 \gamma) \mathrm{e}^{-\gamma / r}\right]^{2}
$$

and notices that the equation $\nabla^{c} R \nabla_{c} R=0$ locating all the possible horizons is equivalent to

$$
\mathrm{e}^{2 \gamma / r}=\frac{2 \gamma-\alpha}{2 \gamma+\alpha}
$$

\footnotetext{
${ }^{6}$ In particular, no wormhole throats exist, contrarily to claims in the literature [176].
} 
The left hand side is never smaller than unity, while the right hand side is always smaller, hence there are no roots and no horizons covering the central singularity. This singularity is timelike. In fact, surfaces of constant radius have normal $N_{\mu}=\nabla_{\mu}\left(r-r_{0}\right)=\delta_{\mu 1}$ with magnitude squared

$$
N^{c} N_{c}=g^{11}=(r / \gamma)^{4} \mathrm{e}^{\alpha / r} \sinh ^{4}(\gamma / r)
$$

which is always positive, therefore $N^{c}$ is spacelike and the surface $r=r_{0}$ is timelike (including in the limit $r_{0} \rightarrow 0$ ).

When $\gamma \neq 0$, by performing the two consecutive coordinate transformations

$$
\mathrm{e}^{\gamma / r}=\frac{1+B / \rho}{1-B / \rho}, \quad \bar{r}=\rho\left(1+\frac{B}{\rho}\right)^{2},
$$

setting $\eta=2 B=\sqrt{m^{2}+\sigma^{2}}, m / \eta=-\alpha /(2 \gamma)$, and rescaling the time coordinate by a factor $|\gamma /(2 B)|$, the FJNWBW solution becomes

$$
\begin{aligned}
d s^{2}= & -\left(1-\frac{2 \eta}{r}\right)^{m / \eta} d t^{2}+\left(1-\frac{2 \eta}{r}\right)^{-m / \eta} d r^{2} \\
& +\left(1-\frac{2 \eta}{r}\right)^{1-m / \eta} r^{2} d \Omega_{(2)}^{2}, \\
\phi(r)= & \frac{\sigma}{2 \eta} \ln \left(1-\frac{2 \eta}{r}\right) .
\end{aligned}
$$

This is the most well-known form of the FJNWBW solution (however, since it requires $\gamma \neq 0$, it is not the most general). Taking this form at face value, one could argue for the existence of non-trivial solutions with nonzero scalar charge $\sigma$ but vanishing mass $m$, as is sometimes done in the literature. However, this possibility is forbidden by the fact that this form of the solution is only valid for $\gamma \neq 0$. The solution in this form is invariant under the symmetry

$$
\begin{aligned}
\frac{m}{\eta} & \rightarrow \frac{m^{\prime}}{\eta^{\prime}}=-\frac{m}{\eta}, \\
r & \rightarrow r^{\prime}=2 \eta-r, \\
\phi & \rightarrow \phi^{\prime}=-\phi .
\end{aligned}
$$


In the special case $\gamma=0$, the FJNWBW metric reduces to the PapapetrouYilmaz or "anti-scalar" geometry ${ }^{7}[178,179,180,181]$

$$
\begin{aligned}
d s^{2} & =-\mathrm{e}^{\alpha / r} d t^{2}+\mathrm{e}^{-\alpha / r}\left(d r^{2}+r^{2} d \Omega_{(2)}^{2}\right), \\
\phi & =\frac{\phi_{0}}{r} .
\end{aligned}
$$

In this case the areal radius is $R(r)=r \mathrm{e}^{-\frac{\alpha}{2 r}}$ and the equation locating the horizons is

$$
\nabla^{c} R \nabla_{c} R=\left(1+\frac{\alpha}{2 r}\right)^{2}=0
$$

If $\alpha>0$ there are no real roots and no horizons, $R(r) \rightarrow 0^{+}$as $r \rightarrow 0^{+}$, and the Ricci scalar

$$
\mathcal{R}=8 \pi \nabla^{c} \phi \nabla_{c} \phi=-8 \pi \phi_{0}^{2} \frac{\mathrm{e}^{\alpha / r}}{r^{4}}
$$

diverges as $R \rightarrow 0^{+}$: there is a naked central singularity. The Misner-SharpHernandez mass $[73,74]$

$$
M_{\mathrm{MSH}}=-\frac{\alpha}{2} \mathrm{e}^{-\frac{\alpha}{2 r}}\left(1+\frac{\alpha}{4 r}\right)
$$

is negative everywhere. If $\alpha<0$, instead, there is only one double root $r_{*}=|\alpha| / 2$ of $\nabla^{c} R \nabla_{c} R=0$, corresponding to a wormhole throat at areal radius $R_{*}=|\alpha| \mathrm{e} / 2$ [177]. In this case, $M_{\mathrm{MSH}}$ is positive for $R>|\alpha| \mathrm{e}^{2} / 4$.

The radial null geodesics of the FJNWBW geometry were studied in [182]. Numerical studies of scalar field collapse generically lead to black holes and not to naked singularities ([183, 184], see ${ }^{8}[144]$ for a review), which leads to seeing the FJNWBW solution as unphysical. As expected from these numerical studies, the FJNWBW geometry is indeed unstable, as shown long ago in [189].

The FJNWBW geometry is the limit of the Garfinkle-Horowitz-Strominger black hole solution [190] of Einstein-Maxwell-dilaton gravity for vanishing mass $M$ and electric charge $Q$, keeping the ratio $Q^{2} / M$ finite, in which the horizon shrinks to a point. An electrovacuum version of the FJNWBW solution of the coupled Einstein-Maxwell-massless scalar field equations was given in [173]. The $d$-dimensional FJNWBW solution for $d \geq 4$ is discussed

\footnotetext{
${ }^{7}$ See [177] for a recent perspective on this "exponential metric".

${ }^{8}$ Critical phenomena occuring in scalar field collapse were discovered by Choptuik in [185] (see also [186, 187] and see [188] for a review).
} 
by Abdolrahimi and Shoom in [191]. Conformal diagrams for the FJNWBW geometry are constructed in [191].

The energy conditions satisfied by the scalar field source of the FJNWBW metric, and the nature of the naked singularity, were discussed in [192]. Gravitational lensing by the FJNWBW naked singularity, including image magnification and time delay between images, were studied in [193, 194, 195].

\subsection{Solutions with $V(\phi) \neq 0$}

No-hair theorems hold for a scalar field $V(\phi)$ that is monotonically increasing and convex [133, 131, 132], but not much is known for other types of self-interaction potential. Allowing for a scalar field potential makes the integration of the field equations more difficult.

Various theorems were formulated which relate the existence of black holes and wormholes with the potential $V(\phi)[134,196,197]$; generally, they place necessary (but not sufficient) restrictions on the shape or sign of $V$ for the existence of black holes and other static spherical solutions. Bronnikov and Fabris [198] classified the regular static and spherically symmetric configurations for canonical and phantom scalar fields with a potential $V(\phi)$. The action is now

$$
S=\int d^{4} x \sqrt{-g}\left[\frac{\mathcal{R}}{2}-\frac{\epsilon}{2} \nabla^{c} \phi \nabla_{c} \phi-V(\phi)\right],
$$

with $\epsilon=+1$ for a canonical and $\epsilon=-1$ for a phantom scalar and static spherical solutions are sought for in the form

$$
d s^{2}=-A(r) d t^{2}+\frac{d r^{2}}{A(r)}+R^{2}(r) d \Omega_{(2)}^{2}, \quad \phi=\phi(r) .
$$

For $\epsilon=+1$, it was shown [198] that $d^{2} R / d r^{2} \leq 0$, which prevents wormholes (because the areal radius $R(r)$ cannot have a positive minimum $R_{0}>0$ ) and stars with finite radius, as well as regular black holes. For $\epsilon=-1$, there is no such restriction and classic wormhole solutions for free scalars [199, 200] are recovered. In total, Bronnikov and Fabris classify the possible regular configurations in sixteen different classes. These include solutions with a regular centre at areal radius $R_{0}$ and asymptotics that are Minkowski, de Sitter, or anti-de Sitter as $R \rightarrow+\infty$ and $r \rightarrow+\infty$; solutions with $-\infty<$ $r<+\infty$ which are wormholes separating two asymptotic spacetime regions; and Kantowski-Sachs homogeneous anisotropic spaces where $A(r)<0$ and the coordinates $t$ and $r$ swap their timelike and spacelike character. 
An example of a phantom solution is given in [198], which is of the form (62) with

$$
\begin{aligned}
& R(r)=\sqrt{r^{2}+b^{2}} \\
& A(r)=R^{2}\left\{\frac{c}{b^{2}}+\frac{1}{R^{2}(r)}+\frac{r_{0}}{b^{3}}\left[\frac{b r}{R^{2}(r)}+\arctan \left(\frac{r}{b}\right)\right]\right\}, \\
& \phi(r)= \pm \sqrt{2} \arctan \left(\frac{r}{b}\right)+\phi_{0},
\end{aligned}
$$

where $b>0, c, r_{0}$, and $\phi_{0}$ are constants. The corresponding potential is not one of the usual physically motivated ones but is constructed ad hoc for this solution and is

$$
\begin{aligned}
V(\phi)= & -\frac{c}{b^{3}}\left[3-2 \cos ^{2}\left(\frac{\phi}{\sqrt{2}}\right)\right] \\
& -\frac{r_{0}}{b^{3}}\left\{\frac{3}{2} \sin (\sqrt{2} \phi)+\frac{\phi}{\sqrt{2}}\left[3-2 \cos ^{2}\left(\frac{\phi}{\sqrt{2}}\right)\right]\right\} .
\end{aligned}
$$

In spite of this rather contrived potential, this is a concrete example of a phantom wormhole solution. The special case $r_{0}=c=0$ gives $A=1$ and $V=0$ and reproduces the Ellis wormhole [199]. The classification of Bronnikov and Fabris [198] extends to more general k-essence theories with Lagrangian $\mathcal{L}=P(X)-V(\phi)$, where $X \equiv-\nabla^{c} \phi \nabla_{c} \phi$ and the regular function $P$ is monotonic.

Another analytic solution of the Einstein-Klein-Gordon equations that is static, spherical, asymptotically flat, and has $\phi \rightarrow 0, V(0)=0, V^{\prime}(0)=$ 0 and $V^{\prime \prime}(0)=0$ was proposed in [201]. It represents a black hole, but the complicated potential $V(\phi)$ has a vertical asymptote where it diverges to minus infinity, which makes the solution unphysical. Furthermore, the asymptotic value $\phi=0$ of the potential is not a minimum but an inflection point. For a particular value of a parameter, this geometry reproduces a previous solution of Anabalón and Oliva [202] which has been shown to be unstable with respect to radial linear perturbations [203].

\subsection{Time-dependent solutions}

In the cases when the solution of the Einstein-scalar field equations is not asymptotically flat or is time-dependent, there is no known general spherical solution and only special solutions are known. We report them in the following. 


\subsubsection{Wyman's "other" solutions}

In addition to the well-known FJNWBW solution, in [170] Wyman presented another class of static spherically symmetric solutions with a massless free scalar field which depends on time. Instead of looking for static solutions with scalar field $\phi=\phi(r)$, the Einstein equations can also be solved by assuming a static metric and $\phi=\phi(t)$, which is what originates Wyman's "other" solutions. In the context of the equivalence between a scalar field and a fluid, this class can be seen as stiff fluid solutions. In most of these solutions, the metric coefficients are given in the form of power series, which leads to formulae too clumsy to be useful in practice (for example, in curvature calculations). The same consideration applies to their generalizations to a non-vanishing cosmological constant given by Varela [204]. However, a particularly simple solution is given explicitly by Wyman [170]:

$$
\begin{aligned}
d s^{2} & =-\kappa r^{2} d t^{2}+2 d r^{2}+r^{2} d \Omega_{(2)}^{2}, \\
\phi(t) & =\phi_{0} t .
\end{aligned}
$$

Substitution into the Einstein equations with a free scalar field as a source yields $\phi_{0}=1 / \sqrt{\kappa}$. This solution was generalized to the case $\Lambda \neq 0$ by Sultana [205] and reproduces a special case of an older family of perfect fluid solutions found by Ibanez and Sanz [206]. The geometry (67) is not asymptotically flat and has a singularity at $r=0$, where the Ricci scalar $\mathcal{R}=$ $8 \pi \nabla^{c} \phi \nabla_{c} \phi=-1 / r^{2}$ and the Ricci squared $\mathcal{R}_{a b} \mathcal{R}^{a b}=1 / r^{4}$ diverge. Since $\nabla^{c} r \nabla_{c} r=1 / 2$, there are no horizons (which are the roots of $\nabla^{c} r \nabla_{c} r=0$ ) and the central singularity is naked. The same conclusions apply to Sultana's generalization [205] with $\Lambda \neq 0$. The Misner-Sharp-Hernandez mass [73, 74] $M_{\mathrm{MSH}}=r / 4$ contained in a sphere of radius $r$ is always positive.

Both Wyman's solution (67), (68) and its generalization by Sultana for $\Lambda \neq 0$ were mapped into the Jordan frame of a conformally coupled scalar field to generate exact solutions, following a technique made popular by Bekenstein for a free scalar and adapted, e.g., by Abreu et al. [207] to the case of a non-zero potential. The same conformal mapping tecnique generates time-dependent spherical solutions of Jordan frame Brans-Dicke gravity (see below) and of $f(\mathcal{R})=\mathcal{R}^{2}$ gravity [208].

The Wyman geometry (67) is reproduced as a special case of a more general static line element obtained by Carloni and Dunsby [209], but for a scalar-tensor theory in which the scalar field is only radius-dependent and is subject to a power-law potential. 
The next two solutions of the Einstein equations describe spherical inhomogeneities embedded in a scalar field-fueled FLRW universe, the first for a free field and the second for exponential potential. They have also been used to generate solutions of scalar-tensor gravity using conformal transformations.

\subsubsection{Husain-Martinez-Nuñez solution}

The scalar field Husain-Martinez-Nuñez (HMN) solution of the Einstein equations [210] is dynamical. It describes a spherical inhomogeneity embedded in a spatially flat FLRW "background" and sourced by a free scalar field. This solution is [210]

$$
\begin{aligned}
d s^{2}= & \left(A_{0} \eta+B_{0}\right)\left[-\left(1-\frac{2 C}{r}\right)^{\alpha} d \eta^{2}+\frac{d r^{2}}{\left(1-\frac{2 C}{r}\right)^{\alpha}}\right. \\
& \left.+r^{2}\left(1-\frac{2 C}{r}\right)^{1-\alpha} d \Omega_{(2)}^{2}\right], \\
\phi(\eta, r)= & \pm \frac{1}{4 \sqrt{\pi}} \ln \left[D\left(1-\frac{2 C}{r}\right)^{\alpha / \sqrt{3}}\left(A_{0} \eta+B_{0}\right)^{\sqrt{3}}\right],
\end{aligned}
$$

where $\eta>0$ is the conformal time of the FLRW "background" (with the Big Bang at $\left.\eta=-B_{0} / A_{0}\right), A_{0}, B_{0}, C$, and $D$ are non-negative constants, and the parameter $\alpha= \pm \sqrt{3} / 2$ can assume only two values. If $A_{0}=0$ the HMN geometry reduces to the FJNWBW one, and the constant $B_{0}$ can be set to zero if $A_{0} \neq 0$, which we do in the following.

The HMN line element is conformal to the FJNWBW one (42), with the scale factor of the "background" FLRW space as the conformal factor, $\Omega=$ $\sqrt{A_{0} \eta}$, and with only two possible values of the Fisher exponent parameter. The sign in Eq. (70) is independent of the sign of $\alpha$. The HMN metric approaches FLRW as $r \rightarrow+\infty$ and it is exactly FLRW if $C=0$ (in which case $A_{0}$ can be eliminated by rescaling $\eta$ ).

There is a central singularity. In fact, both the scalar field $\phi$ and the Ricci scalar

$$
\begin{aligned}
\mathcal{R}= & 8 \pi \nabla^{c} \phi \nabla_{c} \phi=\frac{2 \alpha^{2} C^{2}\left(1-\frac{2 C}{r}\right)^{\alpha-2}}{3 r^{4} A_{0} \eta} \\
& -\frac{3 A_{0}^{2}}{2\left(A_{0} \eta\right)^{3}\left(1-\frac{2 C}{r}\right)^{\alpha}}
\end{aligned}
$$


diverge at $r=2 C$ for both values of $\alpha$, and $r=2 C$ corresponds to areal radius $R=0$. The areal radius is

$$
R(\eta, r)=\sqrt{A_{0} \eta} r\left(1-\frac{2 C}{r}\right)^{\frac{1-\alpha}{2}}
$$

and only the range $2 C \leq r<+\infty$ is physically meaningful, with $r=2 C$ corresponding to areal radius $R=0$.

The HMN line element can be rewritten in terms of the comoving time $t$ of the FLRW "background" as

$$
\begin{aligned}
d s^{2} & =-\left(1-\frac{2 C}{r}\right)^{\alpha} d t^{2}+a^{2}(t) \\
& \times\left[\frac{d r^{2}}{\left(1-\frac{2 C}{r}\right)^{\alpha}}+r^{2}\left(1-\frac{2 C}{r}\right)^{1-\alpha} d \Omega_{(2)}^{2}\right], \\
\phi(t, r) & = \pm \frac{1}{4 \sqrt{\pi}} \ln \left[D\left(1-\frac{2 C}{r}\right)^{\alpha / \sqrt{3}} a^{2 \sqrt{3}}(t)\right],
\end{aligned}
$$

where

$$
a(t)=\sqrt{A_{0} \eta}=a_{0} t^{1 / 3}, \quad a_{0}=\left(\frac{3 A_{0}}{2}\right)^{1 / 3}
$$

(the scale factor dictated by the stiff equation of state $P=\rho$ of a free massless scalar field in a FLRW universe). In terms of the areal radius $R(t, r)=a(t) r A^{\frac{1-\alpha}{2}}(r)$, of a new time coordinate $T$ (introduced to eliminate a cross-term in $d t d R)$, and of $A(r) \equiv 1-2 C / r$, one obtains [47]

$$
\begin{aligned}
& d s^{2}=-A^{\alpha}(r)\left[1-\frac{H^{2} R^{2} A^{2(1-\alpha)}(r)}{B^{2}(r)}\right] F^{2} d T^{2} \\
& +\frac{H^{2} R^{2} A^{2-\alpha}(r)}{B^{2}(r)}\left[1+\frac{A^{1-\alpha}(r)}{B^{2}(r)-H^{2} R^{2} A^{2(1-\alpha)}(r)}\right] d R^{2} \\
& +R^{2} d \Omega_{(2)}^{2},
\end{aligned}
$$

where $B(r) \equiv 1-(\alpha+1) C / r$ and $F$ is an integrating factor guaranteeing that $d T$ is an exact differential. The apparent horizons of the HMN geometry, located by $g^{R R}=0$, are the roots of

$$
B(r)=H(t) R(A(r))^{1-\alpha},
$$


where $r=r(t, R)$, equivalent to [210]

$$
\frac{1}{\eta}=\frac{2}{r^{2}}[r-(\alpha+1) C]\left(1-\frac{2 C}{r}\right)^{\alpha-1} .
$$

If $\alpha=\sqrt{3} / 2$, there exists a critical time $t_{*}$ such that, between the Big Bang and $t_{*}$ there is only one, expanding, apparent horizon. The equation locating the apparent horizons can only be solved numerically and, as a consequence, there is no analytic expression for this time $t_{*}$, which depends on the mass parameter $C$ and on $A_{0}$. At $t=t_{*}$, two additional apparent horizons appear, one of which expands forever, while the other (a black hole apparent horizon) contracts, then merges with the first apparent horizon which in the meantime has been growing. As they meet, these two apparent horizons merge and disappear, causing a timelike naked singularity to appear at $R=0$ in the FLRW "background" [210, 47]. This naked singularity inhabits the universe for the rest of its history and the scalar field is regular on the apparent horizons. This dynamics of apparent horizon radii has been dubbed "S-curve phenomenology" (because of the shape of the horizon radii versus time) and recurs in solutions of GR and of Brans-Dicke theory [47]. The apparent horizons of the HMN solution are spacelike [210, 47].

Apparent horizons appearing or disappearing in pairs constitute a typical phenomenology of time-dependent black holes in GR and in alternative theories of gravity [47]. Geometrically, it can be understood by the fact that the surface $R_{\mathrm{AH}}=R_{\mathrm{AH}}(t)$ defining the apparent horizons can intersect more than once slices of comoving time, producing one, two, or three horizon radii. For some critical value of time, two of these roots coincide and the number of apparent horizons before and after $t_{*}$ differ by two, which is associated with apparent horizons appearing (or disappearing) in pairs.

If $\alpha=-\sqrt{3} / 2$, instead, only one expanding cosmological apparent horizon is present at all times, with a naked singularity at $R=0$.

\subsubsection{Fonarev spacetime}

The Fonarev solution [211] of the coupled Einstein-Klein Gordon equations generalizes the free field Husain-Martinez-Nuñez solution to the case of an exponential potential

$$
V(\phi)=V_{0} \mathrm{e}^{-\lambda \phi},
$$

with $\lambda$ and $V_{0}$ positive constants (this potential has been investigated at length in FLRW cosmology). The Einstein field equations are now

$$
\mathcal{R}_{a b}=8 \pi\left(\nabla_{a} \phi \nabla_{b} \phi+g_{a b} V\right) .
$$


The spherical and time-dependent Fonarev solution is

$$
\begin{aligned}
d s^{2} & =a^{2}(\eta)\left[-f^{2}(r) d \eta^{2}+\frac{d r^{2}}{f^{2}(r)}+S^{2}(r) d \Omega_{(2)}^{2}\right], \\
\phi(\eta, r) & =\frac{1}{\sqrt{\lambda^{2}+2}} \ln \left(1-\frac{2 w}{r}\right)+\lambda \ln a+\frac{1}{\lambda} \ln \left[\frac{V_{0}\left(\lambda^{2}-2\right)^{2}}{2 A_{0}^{2}\left(6-\lambda^{2}\right)}\right],
\end{aligned}
$$

where

$$
\begin{aligned}
& f(r)=\left(1-\frac{2 w}{r}\right)^{\alpha / 2}, \quad \alpha=\frac{\lambda}{\sqrt{\lambda^{2}+2}}, \\
& S(r)=r\left(1-\frac{2 w}{r}\right)^{\frac{1-\alpha}{2}}, \\
& a(\eta)=A_{0}|\eta|^{\frac{2}{\lambda^{2}-2}},
\end{aligned}
$$

with $w$ and $A_{0}$ constants. $\eta$ is the conformal time of the FLRW "background" and we set $A_{0}=1$ for simplicity. In the limit $w \rightarrow 0$, the metric (81) reduces to the spatially flat FLRW geometry with a non-trivial scalar field in the exponential potential (79). In this limit the scalar field reduces to

$$
\phi=\lambda \ln a(t)+\phi_{0}, \quad \phi_{0}=\frac{1}{\lambda} \ln \left[\frac{V_{0}\left(\lambda^{2}-2\right)^{2}}{2 A_{0}^{2}\left(6-\lambda^{2}\right)}\right],
$$

which is equivalent to a perfect fluid with energy density and pressure

$$
\begin{aligned}
& \rho_{(\phi)}=\frac{\dot{\phi}^{2}}{2}+V(\phi)=\frac{\lambda^{2} H^{2}}{2}+\frac{V_{0} \mathrm{e}^{-\lambda \phi_{0}}}{a^{\lambda^{2}}}, \\
& P_{(\phi)}=\frac{\dot{\phi}^{2}}{2}-V(\phi)=\frac{\lambda^{2} H^{2}}{2}-\frac{V_{0} \mathrm{e}^{-\lambda \phi_{0}}}{a^{\lambda^{2}}},
\end{aligned}
$$

and effective dynamical equation of state

$$
w \equiv \frac{P_{(\phi)}}{\rho_{(\phi)}}=\frac{\lambda^{2} H^{2} a^{\lambda^{2}}-2 V_{0} \mathrm{e}^{-\lambda \phi_{0}}}{\lambda^{2} H^{2} a^{\lambda^{2}}+2 V_{0} \mathrm{e}^{-\lambda \phi_{0}}} .
$$

The Friedmann equation $H^{2}=8 \pi \rho / 3$ is then immediately integrated, giving the power law

$$
a(t)=a_{0} t^{2 / \lambda^{2}}
$$


for the scale factor, where

$$
a_{0}=\left[\lambda^{2} \sqrt{\frac{2 \pi V_{0} \mathrm{e}^{-\lambda \phi_{0}}}{3-4 \pi \lambda^{2}}}\right]^{2 / \lambda^{2}}
$$

In the other limit $a \equiv 1$ and $\alpha=1$, the Fonarev geometry becomes the Schwarzschild spacetime (however, one cannot obtain the special value $\alpha=1$ if the condition $\alpha=\frac{\lambda}{\sqrt{\lambda^{2}+2}}$ holds). The Fonarev line element approaches asymptotically a spatially flat FLRW one as $r \rightarrow+\infty$. The Husain-Martinez-Nuñez metric corresponds to the special parameter values $\lambda= \pm \sqrt{6}$ and $V_{0}=0$.

Kastor and Traschen have shown in [212] that the Fonarev geometry arises as a limit of a family of charged dilaton black holes important in string theory $[213,214]$ (indeed, the exponential potential is a trademark of dimensionally reduced theories). Feinstein et al. provide five-dimensional Fonarev spacetimes [215], while higher-dimensional solutions are obtained with a solution-generating technique due to Buchdahl [216] and Tangen [217], see also [218]. ${ }^{9}$

The Fonarev solution contains, during different epochs of the "background" FLRW universe, a naked central singularity or a black hole apparent horizon, and is asymptotically FLRW - it can be thought of as a generalization of the FJNWBW solution (to which it is conformal) to a FLRW background. The physical nature of the Fonarev class of solutions was studied in [211] and [220]. At early times, there is no apparent horizon and there is a naked central singularity embedded in a FLRW "background", but at a critical time (dependent on the parameters) a pair of apparent horizons appears, one expanding (a cosmological horizon) and one contracting and covering a central singularity located at a finite areal radius [211, 220]. The contracting apparent horizon asymptotes to this singularity [211, 220]. Conformal diagrams for the Fonarev spacetime are given in Refs. [211, 220].

A Fonarev solution with a phantom scalar field was discussed in [221]. ${ }^{10}$ It is generated from the Fonarev solution via the transformation $\phi \rightarrow i \phi$,

\footnotetext{
${ }^{9}$ The Buchdahl-Tangen generating technique has been used also to produce higherdimensional versions of the BBMB solution of the conformally coupled-Einstein-Maxwell field equations [219].

${ }^{10} \mathrm{~A}$ phantom field is unstable and should not exist so, at best, it is a signature of a truncated effective theory. Its recent popularity [222, 223, 224, 225, 226] arises from the repeatedly coming and going claims that a phantom equation of state of the dark energy is preferred by cosmological observations.
} 
$\lambda \rightarrow-i \lambda$ which gives the kinetic energy of the scalar the wrong sign in the action and in the field equations. These generalized Fonarev metric and phantom scalar are given by Eq. (81) and

$$
\phi(\eta, r)=\frac{1}{\lambda} \ln \left[\frac{V_{0}\left(\lambda^{2}+2\right)^{2}}{2\left(\lambda^{2}+6\right)}\right]-\lambda \ln a-\frac{1}{\sqrt{\lambda^{2}-2}} \ln \left(1-\frac{2 w}{r}\right),
$$

where now

$$
\begin{aligned}
& f(r)=\left(1-\frac{2 w}{r}\right)^{\alpha / 2}, \quad \alpha=-\frac{\lambda}{\sqrt{\lambda^{2}-2}}, \\
& S(r)=r\left(1-\frac{2 w}{r}\right)^{\frac{1-\alpha}{2}}, \quad a(\eta)=\eta^{-\frac{2}{\lambda^{2}+2}} .
\end{aligned}
$$

Assuming that $\lambda>\sqrt{2}$, the physical meaning of the constant $w$ is recovered as follows. For $\lambda \gg \sqrt{2}$ it is $a \approx 1$ and $\alpha \approx-1$, while [221]

$$
d s^{2} \approx-\left(1-\frac{2 w}{r}\right)^{-1} d \eta^{2}+\left(1-\frac{2 w}{r}\right) d r^{2}+r^{2}\left(1-\frac{2 w}{r}\right)^{2} d \Omega_{(2)}^{2}
$$

The coordinate change [221] $y=r(1-2 w / r)$ brings the line element (95) to the form of the Schwarzschild solution with mass $-w$,

$$
d s^{2}=-\left(1+\frac{2 w}{y}\right) d \eta^{2}+\left(1+\frac{2 w}{y}\right)^{-1} d y^{2}+y^{2} d \Omega_{(2)}^{2},
$$

showing that $w$ corresponds to a negative Misner-Sharp-Hernandez mass $-\left|M_{\mathrm{MSH}}\right|$. The history of the apparent horizons of the phantom Fonarev solution is the time reverse of that of the apparent horizons of the Fonarev spacetime [221].

\subsubsection{Roberts solution and its generalizations}

Let us continue our survey within the context of GR with a canonical, minimally coupled scalar field without mass or potential. The Roberts geometry [227] is a spherical, continuously self-similar spacetime within this context admitting a homothetic Killing vector field $\xi^{c}$ that satisfies $£_{\xi} g_{a b}=2 g_{a b}$. The original form in [227] contained an error later corrected in $[228,229,230,231,232,233]$. It constitutes a useful example in the study of critical phenomena arising in the collapse of scalar fields to black 
holes $[185,229,230,210]$ and it is of interest also for wormhole formation [234, 235, 236, 237, 238, 239]. Its stability was studied by Frolov [240, 241].

Burko [231] corrected and slightly generalized this solution which, in double null coordinates $(u, v, \vartheta, \varphi)$, reads

$$
\begin{aligned}
d s^{2} & =-d u d v+r^{2}(u, v) d \Omega_{(2)}^{2}, \\
r^{2}(u, v) & =\frac{1}{4}\left[\left(1-4 \sigma^{2}\right) v^{2}-2 u v+u^{2}\right] \\
& =\frac{1}{4}[(1-2 \sigma) v-u][(1+2 \sigma) v-u], \\
\phi & = \pm \frac{1}{2} \ln \left[\frac{(1-2 \sigma) v-u}{(1+2 \sigma) v-u}\right],
\end{aligned}
$$

where $\sigma$ is a constant scalar charge. The areal radius squared (98) and the argument of the logarithm in Eq. (100) are positive if $-1 / 2<\sigma<1 / 2$. The limit of vanishing scalar charge $\sigma \rightarrow 0$, in which the matter scalar field $\phi$ disappears, produces Minkowski spacetime. The origin $r=0$ corresponds to $u=(1 \pm 2 \sigma) v$. In the spacetime regions where $\nabla^{c} \phi$ is timelike (in which case the scalar field always corresponds to a perfect fluid), the Roberts solution coincides [231] with a stiff fluid solution of Gutman and Bespal'ko [242].

The trace of the Einstein equations $\mathcal{R}_{a b}=8 \pi \nabla_{a} \phi \nabla_{b} \phi$ yields the Ricci scalar

$$
\mathcal{R}=8 \pi g^{a b} \nabla_{a} \phi \nabla_{b} \phi=-32 \pi \phi_{, u} \phi_{, v}=\frac{8 \pi \sigma^{2} u v}{r^{4}},
$$

which diverges as $r \rightarrow 0$, signaling a spacetime singularity. The latter is naked, as the equation

$$
g^{a b} \nabla_{a} r \nabla_{b} r=-\frac{1}{4 r^{2}}\left[\left(1-4 \sigma^{2}\right) v-u\right](u-v)=0
$$

admits no positive roots (the only roots are $v=u$ or $v=\frac{u}{1-4 \sigma^{2}}$, which give absurdities: if $v=u$, Eq. (98) yields $r^{2}=-\sigma^{2} u^{2}<0$, while $v=u /\left(1-4 \sigma^{2}\right)$ gives $\left.r^{2}=-\frac{\sigma^{2} u^{2}}{1-4 \sigma^{2}}<0\right)$.

The Misner-Sharp-Hernandez mass $[73,74]$ is

$$
M_{\mathrm{MSH}}=\frac{r}{2}\left(1-g^{a b} \nabla_{a} r \nabla_{b} r\right)=-\frac{\sigma^{2} u v}{2 r}
$$

and is negative in the light cone of the origin $u v>0$. Conformal diagrams for the Roberts solution were constructed by de Almeida André [239]. 
Roberts proposed also an anti-de Sitter generalization of his original solution, which is conformal to the previous one [243, 238]. The new field equations

$$
\begin{gathered}
\mathcal{R}_{a b}=2 \Lambda g_{a b}+8 \pi \nabla_{a} \phi \nabla_{b} \phi, \\
\square \phi=0,
\end{gathered}
$$

are solved by $[243,238]$

$$
d s^{2}=\left(1-\frac{\Lambda}{6} u v\right)^{-2}\left[-2 d u d v+f^{2}(u, v) d \Omega_{(2)}^{2}\right],
$$

where

$$
f^{2}(u, v)=-k u v+f_{1} v^{2}+f_{2} u^{2},
$$

where $f_{1,2}$ are constants. If $k^{2}-4 f_{1} f_{2}>0$, the scalar field is [238]

$$
\pm\left(\phi-\phi_{0}\right)=\frac{1}{\sqrt{16 \pi}} \ln \left[\frac{\sqrt{k^{2}-4 f_{1} f_{2}} u+\left(k u-2 f_{1} v\right)}{\sqrt{k^{2}-4 f_{1} f_{2}} u-\left(k u-2 f_{1} v\right)}\right]
$$

for $f_{1} \neq 0$ and

$$
\pm\left(\phi-\phi_{0}\right)=\frac{1}{\sqrt{16 \pi}} \ln \left(f_{2}-k \frac{v}{u}\right)
$$

for $f_{1}=0$, where $\phi_{0}$ is a constant. The Ricci scalar is singular at $f(u, v)=0$, except when $k^{2}-4 f_{1} f_{2}=0$. Also, $v \rightarrow \pm \infty$ and $u \rightarrow \pm \infty$ lead to curvature singularities for $f_{1}=0$ and $f_{2}=0$, respectively, if $k \neq 0$ [238]. Three cases arise:

- When $k^{2}-4 f_{1} f_{2}>0$, the scalar field is real, the equation $f(u, v)=0$ has real roots, and the solution can be parametrized as [238]

$$
f^{2}(u, v)=\frac{1}{4}(a u-v)(b u-v),
$$

where the constants $a$ and $b$ satisfy $a+b=4 k$.

- When $k^{2}-4 f_{1} f_{2}<0$, the scalar field is the phantom given by

$$
\pm\left(\phi-\phi_{0}\right)=i \sqrt{\frac{2}{\kappa^{2}}} \arctan \left(\frac{k u-2 f_{1} v}{\sqrt{4 f_{1} f_{2}-k^{2} u}}\right),
$$

representing a dynamical wormhole [238]. 
- When $k^{2}-4 f_{1} f_{2}=0$, the scalar field $\phi$ is constant and the spacetime is maximally symmetric [238]. It is asymptotically locally AdS with a negative $\Lambda$ and it possesses a black hole event horizon depending on the values of the parameters [238]. In this case, for $k= \pm 1$, the constants $f_{1}$ and $f_{2}$ must be non-negative; for $k=0$, it is either $f_{1}=0$ and $f_{2}>0$, or $f_{2}=0$ and $f_{1}>0[238]$.

Penrose-Carter diagrams for this solution were constructed by Maeda [238] and de Almeida André [239].

\section{Static Kiselev solution}

The static, spherically symmetric Kiselev spacetime [244] has been the subject of a large literature (over 300 articles at the time of writing, which we do not report for lack of space), ranging from null and timelike geodesics to horizon thermodynamics and accretion. This interesting metric is popular as a toy model for the study of various aspects of gravitation. The line element is [244]

$$
\begin{aligned}
d s^{2}= & -\left[1-\frac{2 m}{r}-\sum_{n}\left(\frac{r_{n}}{r}\right)^{3 w_{n}+1}\right] d t^{2} \\
& +\frac{d r^{2}}{1-\frac{2 m}{r}-\sum_{n}\left(\frac{r_{n}}{r}\right)^{3 w_{n}+1}}+r^{2} d \Omega_{(2)}^{2},
\end{aligned}
$$

where $r_{n}>0$ and $-1<w_{n}<-1 / 3$ are constants. The Kiselev spacetime describes a black hole ${ }^{11}$ since it contains an event horizon, a single root of $\nabla^{c} r \nabla_{c} r=g^{r r}=0$, or

$$
r^{3 w_{N}+1}-2 m r^{3 w_{N}}-\sum_{n=1}^{N} r_{n}^{3 w n+1} r^{3\left(w_{N}-w_{n}\right)}=0 .
$$

When the sum $\sum_{n}\left(r_{n} / r\right)^{3 w_{n}+1}$ vanishes, the geometry reduces to Schwarzschild, while the KSdS metric is formally reproduced as $w \rightarrow-1$. The Kiselev metric is not asymptotically flat (because the exponents $\left(3 w_{n}+1\right)$ are negative) and is not asymptotically FLRW, either (if it were, the limit

\footnotetext{
${ }^{11}$ Called a "dirty black hole" in current terminology, because it is surrounded by matter and it Hawking-radiates as a graybody.
} 
$m \rightarrow 0$ would reproduce FLRW space, which is not the case). The Kiselev solution is usually reported as describing a "black hole surrounded by quintessence" but this terminology is extremely misleading. In fact, the matter source is not a perfect fluid (or a mixture of perfect fluids if the sum extends to $n>1$ ) [245] because it exhibits anisotropic pressure. Moreover, the cosmologist's notion of quintessence, ${ }^{12}$ i.e., a perfect fluid with equation of state $P=w \rho$ with $-1<w<-1 / 3$, belongs to accelerated FLRW universes and the Kiselev geometry has no FLRW "background". Unfortunately, most of the literature follows the quintessence interpretation and only recently $([245,247]$, see also $[248,249])$ clarity has been made. ${ }^{13}$ Furthermore, the anisotropic energy-momentum tensor sourcing the Kiselev geometry can be split into a perfect fluid component plus either an electromagnetic or a scalar field [247]. Depending on the parameter values, this stress-energy tensor can violate the null energy condition [247], which means that great care is required in the use of the Kiselev spacetime as a toy model. In the most physically viable of these pictures, an electrically charged fluid is supported by the pressure gradient and by the electric field that it generates [247].

Contrary to what stated in [244], the line element (112) does not contain the Reissner-Nordström metric when the sum $\sum_{n}\left(r_{n} / r\right)^{3 w_{n}+1}$ reduces to $\left(r_{n} / r\right)^{2}$ for a single $w_{q}=1 / 3$. In fact the Reissner-Nordström metric has $g_{00}=-\left(1-\frac{2 m}{r}+\frac{e^{2}}{r^{2}}\right)$ and not $g_{00}=-\left(1-\frac{2 m}{r}-\frac{e^{2}}{r^{2}}\right)$, which is instead what the Kiselev line element (112) reproduces. Moreover, a radiation fluid is not the Kiselev matter source with $w_{q}=1 / 3$ (a value which, strictly speaking, is not allowed by the Kiselev condition $-1<w<-1 / 3$ [244]) and describes an incoherent superposition of electromagnetic waves with random phases, directions of propagation, and polarizations and not the radial electrostatic field of the Reissner-Nordström geometry.

\footnotetext{
${ }^{12}$ Even in cosmology, quintessence is usually an effective perfect fluid consisting of a scalar field $\phi$ in a suitable potential $V(\phi)$. This effective fluid would have a constant equation of state only for very special potentials (for example, two tuned exponential terms [246]), which are not the usual ones encountered in the literature on quintessence. Nevertheless, most articles on the Kiselev black hole keep referring to it as "a black hole surrounded by quintessence" and, in this sense, this terminology is doubly misleading.

${ }^{13}$ The wrong identification of an anisotropic fluid with a perfect one has been all too common in the context of exact fluid spheres in GR [250].
} 
Restricting ourselves to a single component for simplicity, the geometry

$$
d s^{2}=-\left(1-\frac{2 m}{r}-\frac{C}{r^{3 w+1}}\right) d t^{2}+\frac{d r^{2}}{1-\frac{2 m}{r}-\frac{C}{r^{3 w+1}}}+r^{2} d \Omega_{(2)}^{2}
$$

has radial and tangential pressures [245]

$$
\begin{aligned}
& P_{r}=-\rho=-\frac{3 w C}{\kappa r^{3(w+1)}}, \\
& P_{t}=-\frac{3 w(3 w+1)}{2 \kappa r^{3(w+1)}}
\end{aligned}
$$

the pressure anisotropy is [245]

$$
\frac{\Delta P}{\bar{P}}=\frac{3\left(P_{r}-P_{t}\right)}{P_{r}+2 P_{t}}=-\frac{3(w+1)}{2 w}
$$

and the effective average equation of state parameter reads

$$
w \equiv \frac{\bar{P}}{\rho}=\frac{P_{r}+2 P_{t}}{3 \rho} .
$$

This (constant) pressure anisotropy ${ }^{14}$ is always present (except for the KSdS limit $w \rightarrow-1$ ) and clearly shows that the matter source is not a perfect fluid.

The Kiselev metric can be brought to the Kerr-Schild form. Since, for Kerr-Schild metrics, the Einstein equations become linear in Kerr-Schild coordinates [251], the linearity property expressed by the sum $\sum_{n}\left(r_{n} / r\right)^{3 w_{n}+1}$ in the Kiselev line element is not too surprising.

The Misner-Sharp-Hernandez mass contained in a sphere of radius $r$ is

$$
M_{\mathrm{MSH}}=m+\frac{1}{2} \sum_{n} \frac{r_{n}^{3 w_{n}+1}}{r^{3 w_{n}}}=m+\sum_{n} \frac{1}{\left|w_{n}\right|} \frac{4 \pi}{3} r^{3} \rho_{n}(r),
$$

where

$$
8 \pi \rho_{n}=\frac{6\left|w_{n}\right| r_{n}^{3 w_{n}+1}}{r^{3\left(w_{n}+1\right)}}
$$

(a Puiseux expansion [247]). This decomposition is covariant because $M_{\mathrm{MSH}}$ can be expressed as the sectional curvature of the plane tangent to a 2-sphere of symmetry, which is proportional to the Riemann tensor [252].

A pseudo-Newtonian potential for the Kiselev geometry analogous to the Paczinski-Wiita potential for the Schwarzschild spacetime was given in [76].

\footnotetext{
${ }^{14}$ The pressure anisotropy becomes position-dependent if two or more $w$-components are introduced in the matter source [245].
} 


\section{Fluid spheres}

The main motivation for considering fluid spheres in GR is to model (static) relativistic stars. Sometimes it is of interest to consider dynamical asymptotically flat fluid spheres instead of stars. The most common situation occurs in the study of spherical collapse to black holes, but expanding solutions modelling, e.g., exploding wormholes, relativistic fireballs, or explosive events have also been explored [253], although the literature is naturally much smaller than the one on relativistic stellar configurations of equilibrium.

\subsection{Static fluid spheres in GR}

Stars are configurations of equilibrium and the search for static perfect fluid spheres amounts to solving the Einstein equations for hydrostatic and thermodynamic equilibrium. Aside from rotation, spherical symmetry is not just a convenient approximation because static perfect fluid configurations on stellar scales are expected to smooth out "mountains" and other deviations from sphericity (see [254, 255] for a partial proof in GR).

Assuming a static and spherically symmetric geometry of the form

$$
d s^{2}=-\mathrm{e}^{-\Phi(r)} d t^{2}+\frac{d r^{2}}{1-2 m(r) / r}+r^{2} d \Omega_{(2)}^{2}
$$

sourced by a perfect fluid with stress-energy tensor

$$
T_{a b}=(P+\rho) u_{a} u_{b}+P g_{a b},
$$

the fluid four-velocity has components $u^{\mu}=\mathrm{e}^{-\Phi(r)} \delta_{0}^{\mu}$ and

$$
m(r)=4 \pi \int_{0}^{r} d r^{\prime} r^{2} \rho\left(r^{\prime}\right)
$$

is the Misner-Sharp-Hernandez mass [73, 74]. $\Phi(r)$ is determined by the Tolman-Oppenheimer-Volkoff equation of hydrostatic equilibrium [256, 257]

$$
\frac{d \Phi}{d r}-\frac{m(r)+4 \pi r^{3} P(r)}{r^{2}[1-2 m(r) / r]}=0
$$

(see [258] for a discussion of its Newtonian version).

Exact solutions of the Einstein equations in spherical symmetry with perfect fluids as the matter source have a long history, beginning with the toy Schwarzschild interior solution with uniform density [43] now reported 
in all GR textbooks, the Tolman solutions [257], and other classic ones $[259,260,261,262,263,264,265]$. Existence and uniqueness of the solutions are discussed in $[266,267,268]$. While it is possible to find analytic solutions, the simplifying techniques used $[269,44,270]$ very often make the solution rather uninteresting from the physical point of view [250].

Buchdhal [216] proved that all static, spherical perfect fluid stars with $d \rho / d r \leq 0$ satisfy the "Buchdhal bound" $R_{\mathrm{S}} / r<8 / 9$, where $R_{\mathrm{S}}=2 M$ is the Schwarzschild radius and $M$ is the star's mass. Half a century later, Andréasson [271] generalized this bound to the radius-independent upper limit on the compactness $2 m(r) / r<8 / 9$, assuming algebraic inequalities (but not $d \rho / d r \leq 0$ ) on $\rho$ and $P$.

Delgaty and Lake [250], Finch and Skea [270], and Nambo and Sarbach [272] review perfect fluid interior solutions that are static, spherically symmetric, and asymptotically flat. Using computer algebra, Delgaty and Lake [250] classified 127 candidate perfect fluid solutions of the Einstein equations that had appeared in the literature by 1998. They required:

1. isotropic pressure $P(r)$;

2. regularity of the curvature scalars at the origin $r=0$;

3. positive-definite energy density $\rho(0)$ and pressure $P(0)$ at the origin;

4. vanishing pressure $P\left(r_{0}\right)$ at a finite radius $r_{0}>0$ (this is a necessary and sufficient condition to match the interior metric with a Schwarzschild exterior through the usual junction conditions on a spacelike surface $[273]) ;^{15}$

5. the energy density $\rho(r)$ and pressure $P(r)$ are decreasing functions of the physical radius;

6. the adiabatic speed of sound $c_{s}^{2}=d P / d \rho$ is less than the speed of light.

Only 16 candidates satisfied all these physical requirements and, of these, only 9 have monotonically decreasing sound speed [250]. A common failure of satisfying the perfect fluid criterion is that solutions reported as perfect fluid ones in the literature actually have anisotropic pressure instead (in addition, several of the putative solutions reported in the literature do not actually solve the Einstein equations, or were given with typographical errors in the original articles reporting them). As usual with analytic solutions, some of them have been discovered more than once, either because they were

\footnotetext{
${ }^{15}$ Star models with density and pressure vanishing asymptotically as $r \rightarrow+\infty$, which are excluded by this requirement, are also considered to be physical provided that the total mass is finite $[274,275,276]$, which is the case of common boson star models.
} 
disguised by different coordinates (the most common ones are curvature and isotropic coordinates, but Buchdahl, Synge isothermal, and Gaussian polar coordinates have also been used, see [277] for a summary), or because authors were unaware of previous discoveries. ${ }^{16}$

Lake [278] has provided an algorithm to generate all regular static spherically symmetric perfect fluid solutions of the Einstein equations. This algorithm is based on the choice of a single monotonic generating function which is, however, unknown. In order to extract physically meaningful solutions, the generating function must satisfy restrictive integro-differential inequalities expressing physical requirements external to the algorithm [278]. A similar algorithm was proposed in [279]; Martin and Visser [280] proposed a revised version of the Delgaty-Lake algorithm. ${ }^{17}$ Solution-generating theorems are given in $[44,281,282,283,277]$. The general static and spherical perfect fluid solution of the Einstein equations with equation of state $P=-\rho / 5$ was given by Semiz [284] using Buchdahl coordinates.

Given that exact solution catalogues are extensive and evolving, online databases of exact solutions, including perfect fluid spheres, are a better heritage repository than journal articles. While the oldest repositories are now inaccessible, grdb.org stores the metric tensor and performs calculations interactively using the GRTensorII software [285]. Databases and computer algebra are necessarily superseded by new versions.

\subsection{Dynamical fluid spheres in GR}

The search for analytic perfect fluid solutions becomes more difficult when the latter are dynamical. The first step consists of searching for the analogue of the interior Schwarzschild solution with uniform density. Since the interior metric must match the exterior Schwarzschild vacuum, in this case the only possibility for the interior perfect fluid is a dust with vanishing pressure throughout the star [286, 287, 288, 289, 290, 291, 292]. The most well-known solution, which describes the gravitational collapse of a FLRW dust is the one by Oppenheimer and Snyder [286], but other contracting and expanding solutions with uniform density were studied over the years $[293,287,288,289,290,294,295,291,292]$, to which we refer the reader.

Mathematical theorems can be useful not only in the search of exact, but also numerical solutions. For example, it was demonstrated in [296]

\footnotetext{
${ }^{16}$ See Appendix C of [250] for a partial list.

${ }^{17}$ The existence of such algorithms was pointed out by Wyman in 1949, but they were found only relatively recently.
} 
that any arbitrary static anisotropic fluid sphere in GR can be mimicked by a perfect fluid, plus an electromagnetic field, plus a scalar field.

\section{Inhomogeneities embedded in a FLRW universe with fluid}

There are a few spherical and time-dependent solutions of the Einstein equations that describe central objects (which could possibly be black holes, as defined by the notion of apparent horizon instead of event horizon [61, 62, 48]) embedded in FLRW universes. The most well-known is without doubt the $1933 \mathrm{McVittie}$ solution, which has been the subject of renewed attention during the past decade and is also a solution of alternative theories of gravity [297, 298, 299, 300, 301, 302, 303, 304, 305, 306, 307, 308, 309, 310, 311, $312,313,314,315,316,317,318,319,320,321,322]$. The charged version of the McVittie solution is known [323, 324], as well as generalized McVittie solutions [325, 326, 221, 121, 122, 123, 124]. A few other, qualitatively different, solutions are known.

\subsection{McVittie solutions}

In order to study the effect of the cosmic dynamics on local systems, McVittie constructed a family of solutions of the Einstein equations [327] describing a central inhomogeneity embedded in a FLRW universe. Although there have been many studies of this family, mostly recently [328, 329, 297, 330, 331, 252, 301, 305, 332, 311, 312, 333, 308, 309, 303, 306, 307], the latter has proved to be rather complex and it is not yet completely understood in all its aspects. The McVittie spacetime is the only perfect fluid solution of the Einstein equations which is spherically symmetric, shear free, and asymptotically FLRW with no radial energy flow [334]. This theorem is stated explicitly by Raychaudhuri in [334] without giving a formal proof. However, the proof consists of the step-by-step original derivation of this solution by McVittie in [327]. Both electrically neutral and charged McVittie solutions are special cases of the Kustaanheimo-Qvist family of shear-free solutions of the Einstein equations ([335], see also [45]). It has been shown that the McVittie metric cannot be generated as a scalar field solution of the Einstein equations. However, it is a solution of cuscuton theory, a special case of Horava-Lifschitz gravity [316]. The cuscuton theory is the only form of $k$-essence that admits McVittie solutions [316]. The McVittie geometry is also an exact solution of shape dynamics, which constitutes a possible approach to quantum gravity [336], and is also a non-deformable solution of $f(T)$ gravity, where $T$ is the torsion scalar [337]. 
The McVittie line element in isotropic coordinates is

$$
d s^{2}=-\frac{\left(1-\frac{m(t)}{2 \bar{r}}\right)^{2}}{\left(1+\frac{m(t)}{2 \bar{r}}\right)^{2}} d t^{2}+a^{2}(t)\left(1+\frac{m(t)}{2 \bar{r}}\right)^{4}\left(d \bar{r}^{2}+\bar{r}^{2} d \Omega_{(2)}^{2}\right)
$$

where the coefficient $m(t)$ must satisfy the McVittie no-accretion condition $G_{0}^{1}=8 \pi T_{0}^{1}=0$ [327] forbidding radial flow of cosmic fluid and equivalent to

$$
\frac{\dot{m}}{m}+\frac{\dot{a}}{a}=0
$$

which implies $m(t)=m_{0} / a(t)$ with $m_{0}>0$ a constant. Then, the McVittie line element becomes

$$
d s^{2}=-\frac{\left[1-\frac{m_{0}}{2 \bar{r} a(t)}\right]^{2}}{\left[1+\frac{m_{0}}{2 \bar{r} a(t)}\right]^{2}} d t^{2}+a^{2}(t)\left[1+\frac{m_{0}}{2 \bar{r} a(t)}\right]^{4}\left(d \bar{r}^{2}+\bar{r}^{2} d \Omega_{(2)}^{2}\right) .
$$

The McVittie geometry reduces to the Schwarzschild one if $a \equiv 1$, and to the FLRW metric if $m=0$. Apart from the special case of a de Sitter "background", there is a spacetime singularity at $\bar{r}=m / 2$ (this 2 -sphere reduces to the Schwarzschild horizon if $a \equiv 1$ ) [329, 331, 297, 338]. The singularity is spacelike [331, 297]; another singularity occurs at $\bar{r}=0$. McVittie's original interpretation of his geometry (125) as describing a point mass at $\bar{r}=0$ is untenable because, in general, the latter would be surrounded by the second singularity at $\bar{r}=m / 2[338,329,331,297]$, where also the pressure of the cosmic fluid ${ }^{18}$

$$
P=-\frac{1}{8 \pi}\left[3 H^{2}+\frac{2 \dot{H}\left(1+\frac{m}{2 \bar{r}}\right)}{1-\frac{m}{2 \bar{r}}}\right]
$$

and the Ricci scalar $\mathcal{R}=8 \pi(\rho-3 P)$ diverge [338, 329, 331, 297, 339, 340], and the weak and null energy conditions are violated (except in a de Sitter "background").

In terms of the areal radius

$$
R(t, \bar{r}) \equiv a(t) \bar{r}\left(1+\frac{m}{2 \bar{r}}\right)^{2},
$$

\footnotetext{
${ }^{18}$ In the McVittie solution the energy density $\rho$ depends only on time, while the isotropic pressure $P$ depends on both radius and time unless the "background" universe is de Sitter with constant Hubble function $H$, as can be seen from Eq. (128). This radial dependence of $P$ disappears asymptotically far from the central object.
} 
the McVittie line element becomes

$$
d s^{2}=-\left(1-\frac{2 m_{0}}{R}-H^{2} R^{2}\right) F^{2} d T^{2}+\frac{d R^{2}}{1-\frac{2 m_{0}}{R}-H^{2} R^{2}}+R^{2} d \Omega_{(2)}^{2},
$$

where $F(T, R)$ is an integrating factor introduced to guarantee that $d T$ is an exact differential [47] (this becomes unity for a de Sitter "background").

The Misner-Sharp-Hernandez mass of a 2-sphere of radius $R$ is

$$
M_{\mathrm{MSH}}=m_{0}+\frac{H^{2} R^{3}}{2}=m_{0}+\frac{4 \pi}{3} \rho R^{3},
$$

i.e., a time-independent contribution $m_{0}$ from the central object plus the mass of the cosmic fluid, with energy density $\rho$, contained in this sphere.

The apparent horizons of the McVittie metric are well-known. Restricting to the spatially flat case and to dust as an example, the apparent horizons are located by $g^{R R}=0$ or

$$
H^{2}(t) R^{3}-R+2 m_{0}=0 .
$$

This cubic equation is formally the same encountered in the Schwarzschild-de Sitter geometry in static coordinates, but now $H$ is not constant and the apparent horizons are time-dependent. Defining $\psi$ by $\sin (3 \psi) \equiv 3 \sqrt{3} m H$, two horizons exist if $0<\sin (3 \psi)<1$, equivalent to $m_{0} H(t)<1 /(3 \sqrt{3})$, which is only satisfied at certain times. In this flat, dust-dominated "background", only at $t_{*}=2 \sqrt{3} m_{0}$ it is $m_{0} H(t)=1 /(3 \sqrt{3})$. At early times $t<t_{*}$, we have $m_{0}>\frac{1}{3 \sqrt{3} H(t)}$ and no apparent horizons, hence there is a naked singularity. At $t_{*}$, two apparent horizons appear together at $R_{1}=R_{2}=\frac{1}{\sqrt{3} H(t)}$. Later on, as $t>t_{*}$ and $m_{0}<\frac{1}{3 \sqrt{3} H(t)}$, there are two distinct real roots $R_{1,2}(t)$ corresponding to two distinct apparent horizons.

The singularity $R=2 m_{0}$ is spacelike and separates the two spacetime regions $R<2 m_{0}$ and $R>2 m_{0}$ into two disconnected manifolds [331, 297].

An interior solution for the McVittie metric was found by Nolan [330] and describes a relativistic star of uniform density in a FLRW "background". It can be viewed as a generalization of the Schwarzschild interior solution to a FLRW "background". The Nolan line element in isotropic coordinates 
inside this "star" is

$$
\begin{aligned}
d s^{2}= & -\left[\frac{1-\frac{m}{\bar{r}_{0}}+\frac{m \bar{r}^{2}}{\bar{r}_{0}^{3}}\left(1-\frac{m}{4 \bar{r}_{0}}\right)}{\left(1+\frac{m}{2 \bar{r}_{0}}\right)\left(1+\frac{m \bar{r}^{2}}{2 \bar{r}_{0}^{3}}\right)}\right]^{2} d t^{2} \\
& +a^{2}(t) \frac{\left(1+\frac{m}{2 \bar{r}_{0}}\right)^{6}}{\left(1+\frac{m \bar{r}^{2}}{2 \bar{r}_{0}^{3}}\right)^{2}}\left(d \bar{r}^{2}+\bar{r}^{2} d \Omega_{(2)}^{2}\right)
\end{aligned}
$$

where $\bar{r}_{0}$ is the star radius, $m(t)$ satisfies Eq. (126) forbidding accretion onto the star surface, and $0 \leq \bar{r} \leq \bar{r}_{0}$. The interior metric is regular at the centre and satisfies the Darmois-Israel junction conditions at $\bar{r}=\bar{r}_{0}$, matching the exterior McVittie geometry. The pressure is continuous there, while the otherwise uniform energy density is discontinuous [330]:

$$
\begin{aligned}
\rho(t)= & \frac{1}{8 \pi}\left[3 H^{2}+\frac{6 m}{a^{2} \bar{r}_{0}^{3}\left(1+\frac{m}{2 \bar{r}_{0}}\right)^{6}}\right], \\
P(t, \bar{r})= & \frac{1}{8 \pi}\left[-3 H^{2}-2 \dot{H} \frac{\left(1+\frac{m}{2 \bar{r}_{0}}\right)\left(1+\frac{m \bar{r}^{2}}{2 \bar{r}_{0}^{3}}\right)}{1-\frac{m}{\bar{r}_{0}}+\left(1-\frac{m}{4 \bar{r}_{0}}\right) \frac{m \bar{r}^{2}}{\bar{r}_{0}^{3}}}\right. \\
& \left.+\frac{a^{2}\left(1+\frac{m}{2 \bar{r}_{0}}\right)^{6}\left[1-\frac{m}{\bar{r}_{0}}+\left(1-\frac{m}{4 \bar{r}_{0}}\right) \frac{m \bar{r}^{2}}{\bar{r}_{0}^{3}}\right]}{\bar{r}_{0}^{2}}\right] .
\end{aligned}
$$

Like the McVittie solution, the Nolan interior solution belongs to the shearfree family of Kustaanheimo and Qvist [335]. $\rho$ is positive and $P \geq 0$ if $\ddot{a}+3 \dot{a}^{2} / 2<0$. The surface of the star is comoving with the cosmic substratum. The generalized Tolman-Oppenheimer-Volkoff equation inside the star is reported in [325].

Conformal diagrams of the McVittie spacetime were obtained in [305, 332, 311, 312] for various FLRW "backgrounds". These diagrams differ considerably according to the choice of the scale factor $a(t)$ of the FLRW universe in which the central object is embedded. This variety of possibilities has caused some debate in the literature, originating from different choices of $a(t)$ by different authors and from the fact that general statements for all possible behaviours of the scale factor cannot be made. 


\subsubsection{Charged and generalized McVittie geometries}

The charged McVittie solution was introduced by Shah and Vaidya [323] and generalized by Mashhoon and Partovi [324], then rediscovered many years later by Gao and Zhang [341 $]^{19}$ and generalized to higher dimensions [342]. The apparent horizons can be located analytically and explicity in the extremal case and numerically otherwise, revealing that this geometry contains a black hole or a naked singularity according to the values of the mass and charge parameters [314].

The charged McVittie metric was used to disprove the universality of certain quantization laws for quantities built from the areas of black hole apparent horizons and inspired by string theories ${ }^{20}$ [310].

Generalized McVittie solutions allowing for a radial energy flux onto, or from, the central object were introduced in [325]. While these are rather cumbersome, they have a simpler late-time attractor [343] that is recognized as the non-rotating Thakurta [344] solution (see later). A class of solutions of Brans-Dicke gravity found in [345] has the non-rotating Thakurta solution as its limit to general relativity (i.e., the limit in which the Brans-Dicke parameter $\omega \rightarrow \infty$ ) [47].

\subsection{Einstein-Straus, Lemaître-Tolman-Bondi, and Vaidya solutions}

Apparently unaware of the 1933 McVittie solution, Einstein \& Straus approached the problem of cosmic expansion versus local systems more than a decade later, matching a Schwarzschild vacuum to a FLRW universe $[346,347]$ on a finite radius sphere, while the McVittie solution interpolates smoothly between a local dynamical Schwarzschild-like object and the FLRW universe. The Einstein-Straus model is strictly dependent on the spherical symmetry and does not generalize to axisymmetric spacetimes $[348,349,350]$ (see Ref. [351] for a review of the old problem of local dynamics versus cosmic expansion). Nevertheless, it is still used occasionally as a toy model to address cosmological questions [e.g., 352, 353]).

Other well-known spherical and dynamical solutions of the Einstein equations are Lemaître-Tolman-Bondi geometries [354, 355, 288] that can describe the collapse of dust and can be used to address the problem of local versus cosmological dynamics. The Vaidya solutions, intead, describe outgoing or ingoing spherical shells of null dust.

\footnotetext{
${ }^{19}$ Beware of a typographical error in the line element of Gao and Zhang [341].

${ }^{20}$ Beware of the same typographical error present in [341]. The qualitative results are, however, unchanged.
} 
The Einstein-Straus, Lemaître-Tolman-Bondi, and Vaidya solutions of the Einstein equations have been analyzed in detail for decades and we refer the reader to standard books for their discussion [44, 45, 46].

\subsection{Solutions generated by conformal transformations}

Certain spherically symmetric and time-dependent solutions of the Einstein equations representing central objects embedded in FLRW universes are generated by conformally transforming Schwarzschild, with the scale factor of the FLRW "background" universe as the conformal factor. These solutions include the non-rotating Thakurta [344] solution, the Sultana-Dyer [356], and the McClure-Dyer solutions [339, 340, 357]. The matter sources are usually complicated and it is common to have spacetime regions with negative energy densities, making these regions unphysical. However, some of these geometries may turn out to be acceptable in other theories of gravity, and they could perhaps be acceptable solutions in GR with different matter sources (an issue that has not yet been investigated).

\subsubsection{Non-rotating Thakurta spacetime}

The non-rotating Thakurta solution [344] is the zero angular momentum limit of the GR solution originally introduced to model a Kerr black hole embedded in a FLRW universe. The line element is conformal to Schwarzschild,

$$
\begin{aligned}
d s^{2} & =a^{2}(\eta)\left[-\left(1-\frac{2 m}{r}\right) d \eta^{2}+\frac{d r^{2}}{1-2 m / r}+r^{2} d \Omega_{(2)}^{2}\right] \\
& =-\left(1-\frac{2 m}{r}\right) d t^{2}+\frac{a^{2} d r^{2}}{1-2 m / r}+a^{2} r^{2} d \Omega_{(2)}^{2},
\end{aligned}
$$

where $a(\eta)$ is the scale factor of the FLRW "background", $\eta$ and $t$ are its conformal and comoving times, respectively, with $d t=a d \eta$, and $m$ is a constant mass parameter. One can switch to the areal radius $R(\eta, r)=a(\eta) r$ and the new time coordinate $T$ defined by

$$
d T=\frac{1}{F}\left(d t+\frac{H R}{A^{2}-H^{2} R^{2}} d R\right)
$$

where $F(t, R)$ is an integrating factor satisfying

$$
\frac{\partial}{\partial R}\left(\frac{1}{F}\right)=\frac{\partial}{\partial t}\left(\frac{H R}{F\left(A^{2}-H^{2} R^{2}\right)}\right)
$$


to guarantee that $d T$ is an exact differential, recasting the Thakurta line element in the form $[319,125]$

$$
d s^{2}=-\left(1-\frac{2 M}{R}-\frac{H^{2} R^{2}}{1-\frac{2 M}{R}}\right) F^{2} d T^{2}+\frac{d R^{2}}{1-\frac{2 M}{R}-\frac{H^{2} R^{2}}{1-2 M / R}}+R^{2} d \Omega_{(2)}^{2},
$$

where $A(t, R)=1-2 M / R$ and $M(t) \equiv m a(t)$. Using the form (136) of the metric, the Einstein equations give

$$
\begin{aligned}
G_{0}{ }^{0}=8 \pi T_{0}^{0} & =-\frac{3 H^{2}}{1-2 M / R}, \\
G_{1}{ }^{0}=8 \pi T_{1}^{0} & =-\frac{2 m H}{r^{2}(1-2 M / R)^{2}}, \\
G_{1}{ }^{1}=8 \pi T_{1}^{1} & =8 \pi T_{2}^{2}=8 \pi T_{3}^{3} \\
& =-\frac{1}{1-2 M / R}\left(H^{2}+\frac{2 \ddot{a}}{a}\right) .
\end{aligned}
$$

Since $T^{0}{ }_{r} \neq 0$, there is a radial energy flow with spacelike current density ${ }^{21}$ $q_{\mu}=\left(0,-2 m \dot{a} a(1-2 M / R)^{-3 / 2} / r^{2}, 0,0\right)$ in coordinates $(t, r, \vartheta, \varphi)$ [319], therefore the matter source is an imperfect fluid.

The non-rotating Thakurta solution is the late-time attractor of generalized McVittie solutions ${ }^{22}$ [343, 47]. The non-rotating Thakurta solution is also the limit to GR of the Clifton-Mota-Barrow family of solutions of Brans-Dicke theory [345] as the Brans-Dicke parameter $\omega \rightarrow \infty[343,47]$. The non-rotating Thakurta solution was studied in detail in [319] (see also $[358,359])$. Conformal diagrams for the non-rotating Thakurta solution can be found in Ref. [319] for various choices of the scale factor $a(t)$.

The non-rotating Thakurta solution has been used [41], together with the class of generalized McVittie solutions of which it is an attractor [42], as a toy model for primordial black holes for which the size of the black hole

\footnotetext{
${ }^{21}$ This current density is spacelike and non-causal, as in the imperfect fluid stress-energy tensor $T_{a b}=(P+\rho) u_{a} u_{b}+P g_{a b}+q_{a} u_{b}+q_{b} u_{a}$, or in Eckart's first order thermodynamics for dissipative fluids, which are non-causal.

${ }^{22}$ The authors of $[47,343,358]$ did not recognize this geometry as the non-rotating Thakurta solution.
} 
horizon is not completely negligible in comparison with the Hubble radius (the latter can be very small in the early universe). The time-dependent black hole apparent horizon is not a null event horizon and the MisnerSharp-Hernandez mass of this black hole is time-dependent, contrary to the Schwarzschild mass. These differences with respect to Schwarzschild could be important for the evolution of primordial black holes, which can in principle account for a substantial fraction (or, hypothetically, even all) of dark matter, according to an old hypothesis that is seeing a new lease on life due to the unexpectedly large black hole masses measured in black hole mergers by the LIGO/VIRGO detectors [39, 40].

\subsubsection{Sultana-Dyer spacetime}

The Sultana-Dyer solution of the Einstein equations [356] is a Petrov type D geometry whose physical interpretation, according to their proponents, is a black hole embedded in a spatially flat FLRW universe. The scale factor of this FLRW universe is $a(t) \propto t^{2 / 3}$ in comoving time, i.e., that associated with a dust. This solution is "manufactured" by conformally rescaling the Schwarzschild metric, $g_{a b}^{(S)} \rightarrow g_{a b}=\Omega^{2} g_{a b}^{(S)}$. The explicit intention of Sultana \& Dyer was to map the timelike Killing field $\xi^{c}$ of Schwarzschild into a conformal Killing field (which requires $\xi^{c} \nabla_{c} \Omega \neq 0$ ), and the Schwarzschild event horizon into a conformal Killing horizon. The line element in its original form is obtained by conformally transforming the Schwarzschild metric [356]

$$
d s^{2}=a^{2}(\eta)\left[-\left(1-\frac{2 m}{\tilde{r}}\right) d \eta^{2}+\frac{4 m}{\tilde{r}} d \eta d \tilde{r}+\left(1+\frac{2 m}{\tilde{r}}\right) d \tilde{r}^{2}+\tilde{r}^{2} d \Omega^{2}\right]
$$

with $m$ a constant mass parameter ${ }^{23}$ and $a(\eta)=a_{0} \eta^{2}=a_{0} t^{2 / 3}$.

The Sultana-Dyer metric can be rewritten using the new time defined by

$$
d t=d \bar{t}+\frac{2 m a d \tilde{r}}{\tilde{r}\left(1-\frac{2 m}{\tilde{r}}\right)}
$$

\footnotetext{
${ }^{23}$ The metric signature of [356] is opposite to ours and their notation for $\eta$ and $t$ is switched with respect to ours.
} 
which transforms the line element (143) into

$$
\begin{aligned}
d s^{2} & =-\left(1-\frac{2 m}{\tilde{r}}\right) d \vec{t}^{2}+\frac{a^{2} d \tilde{r}^{2}}{1-\frac{2 m}{\tilde{r}}}+a^{2} \tilde{r}^{2} d \Omega^{2} \\
& =a^{2}\left[-\left(1-\frac{2 m}{\tilde{r}}\right) d \bar{\eta}^{2}+\frac{d \tilde{r}^{2}}{1-\frac{2 m}{\tilde{r}}}+\tilde{r}^{2} d \Omega^{2}\right] .
\end{aligned}
$$

This is explicitly conformal to Schwarzschild written in Schwarzschild coordinates, with conformal factor $a$ and $d \bar{t}=a d \bar{\eta}$, and it approaches the Einstein-de Sitter geometry as $\tilde{r} \rightarrow+\infty$.

The matter source of the Sultana-Dyer metric is a mixture of two noninteracting perfect fluids, a null dust and a massive dust [356], with total energy-momentum tensor

$$
T_{a b}=T_{a b}^{(\mathrm{I})}+T_{a b}^{(\mathrm{II})}=\rho u_{a} u_{b}+\rho_{n} k_{a} k_{b},
$$

and $k^{c} k_{c}=0, k^{c} \nabla_{c} k_{a}=0[356]$.

The Ricci scalar

$$
\mathcal{R}=\frac{12}{\tilde{\eta}^{6}}\left(1-\frac{2 m}{\tilde{r}}+\frac{2 m \tilde{\eta}}{\tilde{r}^{2}}\right)
$$

diverges at the central spacetime singularity $\tilde{r}=0$ and at the Big Bang $\tilde{\eta}=0$. The equation $\nabla^{c} R \nabla_{c} R=0$ (where $R$ is the areal radius) locating the apparent horizons reads

$$
2 m a+\frac{\tilde{r}^{2} a_{, \tilde{r}}^{2}}{a}\left(1+\frac{2 m}{\tilde{r}}\right)-4 m \tilde{r} a_{, \tilde{r}}-a \tilde{r}=0
$$

or, since $a(t)=t^{2}[356,360,47]$,

$$
4 \tilde{r}^{3}+8 m \tilde{r}^{2}-(8 m+t) t \tilde{r}+2 m t^{2}=0 .
$$

The positive roots of this cubic, expressed in terms of the areal radius $R=$ $a \tilde{r}$, are $[356,360,47]$

$$
\begin{aligned}
& R_{1}(t)=\frac{t^{3}}{2} \\
& R_{2}(t)=2 m t^{2}, \\
& R_{3}(t)=-m-\frac{t}{4}+\frac{t^{2}}{4} \sqrt{t^{2}+24 m t+16 m^{2}} .
\end{aligned}
$$


$R_{1}$ is the radius of a cosmological apparent horizon; $R_{2}$ is the areal radius of the conformally transformed Schwarzschild event horizon which, being a null surface, remains an event horizon for the Sultana-Dyer solution; and $R_{3}$ is the radius of an apparent horizon. The Sultana-Dyer construct describes a black hole hiding a central spacetime singularity below its horizons [356, $360,47]$. Conformal diagrams for the Sultana-Dyer geometry are given in Ref. [360].

\subsection{Other solutions}

There have been other attempts to construct time-dependent black holes by conformally transforming the Schwarzschild geometry. These works have generated the McClure-Dyer and similar solutions of the Einstein equations $[339,340,357,358]$. Other inhomogeneous spherical solutions of the Einstein equations include the Abbassi-Meissner [118, 119, 120] and the Castelo Ferreira $[122,124,121,123]$ geometries. The former is a fluid solution with radial mass flow and the latter is generated by a fluid with anisotropic pressure and contains the McVittie metric as a special case. They are analyzed in [125]. Other spherical solutions proposed in the literature include those of Carr et al. [361], Chakrabarti and Banerjee [362], Firouzjaee and Mansouri [363], Kastor and Traschen [364], and Sun [365].

\section{Part II}

\section{Spherical solutions of scalar-tensor gravities}

Scalar-tensor gravity is the prototypical alternative to GR and contains the popular class of $f(\mathcal{R})$ gravities. The salient feature is that the gravitational coupling constant is promoted to the role of a dynamical scalar field. Scalar-tensor gravity, in particular $f(\mathcal{R})$ gravity, is used as an alternative to dark energy to explain the current acceleration of the universe. Theories that achieve this goal contain a time-dependent effective cosmological "constant" and it is interesting to understand how black holes in these theories look like. If stationary and asymptotically flat, they are the same as GR black holes (apart from mavericks), otherwise they are time-dependent and characterized by apparent (instead of event) horizons, a subject of considerable interest already in GR. Another motivation to study analytic inhomo-

geneous solutions of scalar-tensor gravity is exploring the spatial variation of 
fundamental constants, in this case the gravitational coupling. Furthermore, there is hope that deviations from GR may be detectable at astrophysical scales in processes involving strong gravity near black holes. Many theoretical efforts are devoted to the possibility of detecting black hole hair, which is forbidden in vacuum GR $[159,160,129,366,367,130,162]$ but not in the presence of matter or in other theories of gravity [368, 141, 369, 140]. Finally, bosonic string theory reduces to an $\omega=-1$ Brans-Dicke theory in its low-energy limit [370, 371], and Brans-Dicke spacetimes are often very similar to exact solutions of dilaton gravity.

\section{Scalar-tensor gravity}

The Jordan frame scalar-tensor gravity action is $[29,53,54,55]$

$$
S_{\mathrm{ST}}=\frac{1}{2 \kappa} \int d^{4} x \sqrt{-g}\left[\phi \mathcal{R}-\frac{\omega(\phi)}{\phi} \nabla^{c} \phi \nabla_{c} \phi-V(\phi)\right]+S^{(\mathrm{m})},
$$

where $\phi>0$ is the Brans-Dicke scalar (with the effective gravitational coupling approximately equivalent to $\phi^{-1}$ ), the function $\omega(\phi)$ is the "BransDicke coupling", $V(\phi)$ is a scalar field potential, and $S^{(\mathrm{m})}=\int d^{4} x \sqrt{-g} \mathcal{L}^{(\mathrm{m})}$ is the matter action.

The variation of the action (153) produces the (Jordan frame) field equations $[29,53,54,55]$

$$
\begin{aligned}
\mathcal{R}_{a b}-\frac{1}{2} g_{a b} \mathcal{R}= & \frac{8 \pi}{\phi} T_{a b}^{(\mathrm{m})}+\frac{\omega}{\phi^{2}}\left(\nabla_{a} \phi \nabla_{b} \phi-\frac{1}{2} g_{a b} \nabla_{c} \phi \nabla^{c} \phi\right) \\
& +\frac{1}{\phi}\left(\nabla_{a} \nabla_{b} \phi-g_{a b} \square \phi\right)-\frac{V}{2 \phi} g_{a b}, \\
\square \phi= & \frac{1}{2 \omega+3}\left(\frac{8 \pi T^{(\mathrm{m})}}{\phi}+\phi \frac{d V}{d \phi}-2 V-\frac{d \omega}{d \phi} \nabla^{c} \phi \nabla_{c} \phi\right),
\end{aligned}
$$

where $T^{(\mathrm{m})} \equiv g^{a b} T_{a b}^{(\mathrm{m})}$ is the trace of the matter stress-energy tensor $T_{a b}^{(\mathrm{m})}$. The original Brans-Dicke theory [29] had $\omega=$ const. and no scalar field potential. 
By performing the conformal transformation of the metric and redefining non-linearly the Brans-Dicke-like scalar as in

$$
\begin{aligned}
& g_{a b} \rightarrow \tilde{g}_{a b}=\Omega^{2} g_{a b}, \quad \Omega=\sqrt{\phi}, \\
& d \tilde{\phi}=\sqrt{\frac{|2 \omega(\phi)+3|}{2 \kappa}} \frac{d \phi}{\phi}
\end{aligned}
$$

for $\omega \neq-3 / 2$, the scalar-tensor action assumes the Einstein frame form

$$
S_{\mathrm{ST}}=\int d^{4} x \sqrt{-\tilde{g}}\left[\frac{\tilde{\mathcal{R}}}{2 \kappa}-\frac{1}{2} \tilde{g}^{a b} \tilde{\nabla}_{a} \tilde{\phi}_{\nabla_{b}} \tilde{\phi}-U(\tilde{\phi})+\frac{\mathcal{L}^{(\mathrm{m})}}{(\phi)^{2}}\right]
$$

where a tilde denotes quantities associated with the rescaled metric $\tilde{g}_{a b}, \mathcal{L}^{(\mathrm{m})}$ is the matter Lagrangian density, and

$$
U(\tilde{\phi})=\frac{V[\phi(\tilde{\phi})]}{[\phi(\tilde{\phi})]^{2}}
$$

This is formally the action of GR with a minimally coupled scalar field $\tilde{\phi}$, but with the important difference that this scalar now couples explicitly to matter. The Einstein frame field equations are

$$
\begin{aligned}
& \tilde{\mathcal{R}}_{a b}-\frac{1}{2} \tilde{g}_{a b} \tilde{\mathcal{R}}=\frac{8 \pi}{\phi} T_{a b}^{(\mathrm{m})}+\tilde{T}_{a b}^{(\tilde{\phi})}, \\
& \tilde{\square} \tilde{\phi}-\frac{d U}{d \tilde{\phi}}=\frac{8 \pi T^{(\mathrm{m})}}{\phi^{2}},
\end{aligned}
$$

where

$$
\tilde{T}_{a b}^{(\tilde{\phi})}=\tilde{\nabla}_{a} \tilde{\phi} \tilde{\nabla}_{b} \tilde{\phi}-\frac{1}{2} \tilde{g}_{a b} \tilde{g}^{c d} \tilde{\nabla}_{c} \tilde{\phi} \tilde{\nabla}_{d} \tilde{\phi}-\frac{U(\tilde{\phi})}{2} \tilde{g}_{a b}
$$

is the canonical stress-energy tensor for a scalar field minimally coupled with the curvature. If the metric $g_{a b}$ is of the spherically symmetric form considered below, also the rescaled $\tilde{g}_{a b}$ assumes the same form with $\Omega(\phi)=$ $\Omega(t, r)$.

A widely known solution-generating technique in scalar-tensor gravity (usually attributed to Bekenstein but anticipated before him [372, 169]) 
consists of taking a scalar field solution of GR and regarding it as the Einstein frame version of a Jordan frame counterpart, then mapping it back to the Jordan frame. In general, a scalar field potential that is physically well motivated in the Einstein frame gives rise to a meaningless one in the Jordan frame, however this technique works in vacuo and for certain potentials.

A lesser known solution-generating technique restricted to Brans-Dicke gravity with electrovacuum or with conformally invariant matter relies on a symmetry of the Brans-Dicke field equations. By taking a solution of this theory as a seed, one applies the symmetry [373, 374]

$$
\begin{aligned}
g_{a b} & \rightarrow \hat{g}_{a b}=\phi^{2 \alpha} g_{a b}, \\
\phi & \rightarrow \hat{\phi}=\phi^{1-2 \alpha}, \\
\omega & \rightarrow \hat{\omega}(\omega, \alpha)=\frac{\omega+6 \alpha(1-\alpha)}{(1-2 \alpha)^{2}},
\end{aligned}
$$

of the corresponding Brans-Dicke field equations for $\alpha \neq 1 / 2$ to generate a new solution. ${ }^{24}$ Since, as $\alpha$ varies, there is a one-parameter Abelian group of transformations $[373,374]$, the result is a family of solutions with an extra parameter $\alpha$.

\subsection{Black holes in scalar-tensor gravity: no-hair theorems}

All asymptotically flat, stationary, axially symmetric black holes of physical relevance in vacuum scalar-tensor gravity reduce to GR black holes. A theorem [163] states that all vacuum, stationary, and asymptotically flat black holes of Brans-Dicke gravity (i.e., with $\omega=$ const. and $V(\phi) \equiv 0$ ) must be Kerr black holes. The proof, performed in the Einstein frame, does not go through the direct integration of the Brans-Dicke field equations, but uses an integral technique. It can be repeated by allowing the coupling $\omega$ to be a function of $\phi$. A previous result of Hawking [162] states that a stationary, asymptotically flat black hole in vacuum GR is necessarily axisymmetric and must have spherical topology, if the weak or the null energy conditions hold. The theorem by Hawking [163] extends this earlier result to Brans-Dicke theory proving that the Brans-Dicke scalar field is static. The advantage of using the Einstein frame is that the rescaled Brans-Dicke

\footnotetext{
${ }^{24}$ The conformal transformation (163) has nothing to do with the conformal relation between Jordan and Einstein frames.
} 
scalar

$$
\tilde{\phi}=\sqrt{\frac{|2 \omega+3|}{2 \kappa}} \ln \left(\frac{\phi}{\phi_{*}}\right)
$$

(where $\phi_{*}$ is a constant) satisfies the weak and null energy conditions [163]. Since the spacetime is stationary, it is also axisymmetric [162]. Outside the horizon (with radius $r_{\mathrm{H}}$ ), there exist a timelike Killing field $t^{a}$ and a spacelike Killing field $\psi^{a}$ and the Einstein frame scalar $\tilde{\phi}$ is constant along the orbits of $t^{a}$ and $\psi^{a}$. Therefore, for $r>r_{\mathrm{H}}, \nabla^{a} \tilde{\phi}$ can only be spacelike or zero. Consider a four-dimensional volume $\mathcal{V}$ bounded by two Cauchy hypersurfaces $\mathcal{S}$ and $\mathcal{S}^{\prime}$ representing two consecutive instants of time $t$ (where $\left.t^{a} \equiv(\partial / \partial t)^{a}\right)$, a portion of the black hole event horizon $H$, and spatial infinity $i^{0}$ [163] (fig. 1). The Einstein frame field equation (161) for $\tilde{\phi}$ in vacuo and with

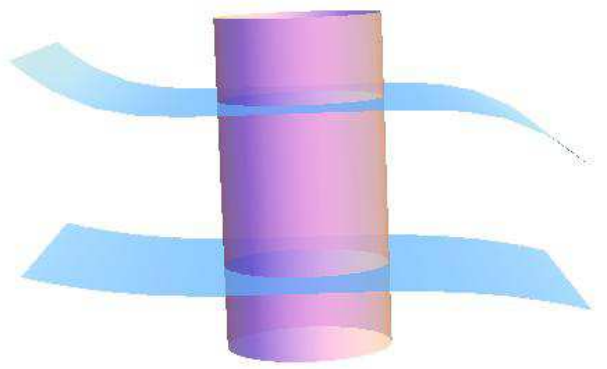

Figure 1: The four-dimensional volume $\mathcal{V}$ bounded by the Cauchy hypersurfaces $\mathcal{S}$ and $\mathcal{S}^{\prime}$, the black hole event horizon $H$, and spatial infinity $i^{0}$.

$V=0$ becomes $\tilde{\square} \tilde{\phi}=0$. Multiplying this equation by $\tilde{\phi}$, integrating over $\mathcal{V}$, and using the Gauss theorem and the identity $\tilde{\phi} \tilde{\square} \tilde{\phi}=\tilde{\nabla}^{c}\left(\tilde{\phi}_{\bar{\nabla}} \tilde{\phi}\right)-\tilde{\nabla}^{c} \tilde{\phi} \tilde{\nabla}_{c} \tilde{\phi}$, one obtains [163]

$$
\int_{\mathcal{V}} d^{4} x \tilde{g}^{a b} \tilde{\nabla}_{a} \tilde{\phi} \tilde{\nabla}_{b} \tilde{\phi}=\int_{\partial \mathcal{V}} d S^{c}\left(\tilde{\phi} \tilde{\nabla}_{c} \tilde{\phi}\right)
$$


The integral over the boundary $\partial \mathcal{V}$ on the right hand side is split into four contributions:

$$
\int_{\partial \mathcal{V}} d S^{c}\left(\tilde{\phi} \tilde{\nabla}_{c} \tilde{\phi}\right)=\left(\int_{\mathcal{S}}+\int_{\mathcal{S}^{\prime}}+\int_{i^{0}}+\int_{H}\right) d S^{c}\left(\tilde{\phi} \tilde{\nabla}_{c} \tilde{\phi}\right) .
$$

The integrals over the Cauchy hypersurfaces $\mathcal{S}$ and $\mathcal{S}^{\prime}$ cancel out because they have the same absolute value due to time symmetry but opposite signs because of the opposite directions that the outgoing unit normal has on these hypersurfaces. The integral over $i^{0}$ vanishes because $\tilde{\nabla}_{a} \tilde{\phi}$ vanishes there. ${ }^{25}$ Finally, the integral over the portion of the horizon $H$ vanishes because the projection of $\tilde{\nabla}_{a} \tilde{\phi}$ along the null vector tangent to the horizon, which is a linear combination of $t^{a}$ and $\psi^{a}$, vanishes due to the symmetries [163]. This argument fails if the Einstein frame scalar $\tilde{\phi}$ or its gradient $\tilde{\nabla}_{c} \tilde{\phi}$ are not defined at the horizon. This is the case of maverick solutions violating the Hawking theorem, in which $\tilde{\phi} \propto \ln \phi$ diverges on the horizon because $\phi$ vanishes or diverges there; then the conformal transformation becomes ill-defined at the horizon and the Einstein frame variables $\left(\tilde{g}_{a b}, \tilde{\phi}\right)$ cannot be used on this hypersurface. However, these situations are unphysical.

Hawking's theorem [163] has been generalized, with the limitation of spherical symmetry, to various scalar-tensor theories with varying $\omega[137$, $134,149,375]$ and with potential $V(\phi)$, provided that $V$ has a minimum at the constant value $\phi_{0}$ at which the Brans-Dicke scalar stabilizes outside the horizon, so that the dynamics of $\phi$ stops there [376]. A generalization to asymptotically de Sitter spaces was given by Bhattacharya et al. [377]. Assuming again spherical symmetry, Hawking's theorem can be proved also in the Jordan frame [378]. Indeed, numerical studies of black hole collapse in Brans-Dicke [379, 380] and scalar-tensor gravity [381, 382] find the Schwarzschild black hole as the final state.

No-hair theorems for more general Horndeski and Galileon theories, and ways to evade them, are the subject of a large literature $([383,384,141,140]$ and references therein) (see Sec. 13).

Weak forms of the Jebsen-Birkhoff theorem, analogous to those of [97, $98,99,100]$ in GR, state that the geometry is static if the scalar field $\phi$ (or its effective stress-energy tensor) is static [385, 386, 378]. An error in Ref. [385] is corrected in [386] and the extension of these results to cylindrical and planar symmetries is studied by Bronnikov and Kovalchuk [100].

\footnotetext{
${ }^{25}$ It is here that the assumption of asymptotic flatness becomes crucial.
} 


\section{The general non-black hole, spherical, static, asymptotically flat solution}

Under the assumptions that: 1) the vacuum Brans-Dicke field equations (with $\omega \neq-3 / 2$ ) hold in the Jordan frame; 2) the geometry is spherically symmetric, static, and asymptotically flat; 3) the massless Brans-Dicke scalar $\phi$ depends only on the radial coordinate $r$, does not have poles or zeros (except possibly for a central singularity), and $\phi(r)$ becomes constant as $r \rightarrow+\infty$, the general solution that is not a Schwarzschild black hole is known $[200,387]$ and its nature depends on a scalar charge parameter. The only possibilities for the nature of these spacetimes (apart from the Schwarzschild black hole) are wormhole throats and central naked singularities. This is the closest known result to the Birkhoff theorem of GR in scalar-tensor gravity.

The history of this general solution is a bit confused. A theorem of Agnese and La Camera [388] stated that the possible solutions describe only wormholes or naked singularities, but it is wrong as this would exclude the Schwarzschild black hole and contradict Hawking's no-hair theorem and its generalizations. The error of Agnese and La Camera [388] consists of assuming the line element and scalar field as

$$
\begin{aligned}
d s_{\mathrm{ALC}}^{2}= & -\left(1-\frac{2 \eta}{r}\right)^{A} d t^{2}+\left(1-\frac{2 \eta}{r}\right)^{B} d r^{2} \\
& +\left(1-\frac{2 \eta}{r}\right)^{1+B} r^{2} d \Omega_{(2)}^{2}, \\
\phi_{\mathrm{ALC}}(r)= & \phi_{0}\left(1-\frac{2 \eta}{r}\right)^{\frac{-(A+B)}{2}},
\end{aligned}
$$

with

$$
1-\frac{\omega+1}{\omega+2}=\frac{(A+B)^{2}}{2(1+A B)}
$$

where $A, B$, and $\eta$ are real constants. This is assumed to be a permissible gauge choice in [388] but it is instead a specific solution, which nowadays is called the Campanelli-Lousto solution. The general form of the Campanelli- 
Lousto solution is $[389,390]$

$$
\begin{aligned}
d s_{\mathrm{CL}}^{2}= & -\left(1-\frac{2 \eta}{r}\right)^{b_{0}+1} d t^{2}+\left(1-\frac{2 \eta}{r}\right)^{-a_{0}-1} d r^{2} \\
& +\left(1-\frac{2 \eta}{r}\right)^{-a_{0}} r^{2} d \Omega_{(2)}^{2}, \\
\phi_{\mathrm{CL}}(r)= & \phi_{0}\left(1-\frac{2 \eta}{r}\right)^{\frac{a_{0}-b_{0}}{2}},
\end{aligned}
$$

where the parameters $a_{0}$ and $b_{0}$ satisfy

$$
\omega=\frac{-2\left(a_{0}^{2}+b_{0}^{2}-a_{0} b_{0}+a_{0}+b_{0}\right)}{\left(a_{0}-b_{0}\right)^{2}} .
$$

The values $a_{0}=-B-1$ and $b_{0}=A-1$ reproduce the Agnese-La Camera choice. Therefore, their results can only be true for this particular solution, which resolves the conflict with the no-hair theorems. The CampanelliLousto solution was originally advertised as a family of black holes [389, 390], but it describes only wormhole throats or naked singularities instead [391].

The general solution was instead obtained by Bronnikov [200] (see also ${ }^{26}$ [387]). The main idea consists of going to the Einstein frame, in which the Brans-Dicke scalar field becomes a standard, minimally coupled, massless, matter scalar field and the general solution is well-known to be the FJNWBW metric (42), (43) of GR. The general Jordan frame solution is then obtained by inverting the conformal transformation from the Jordan to the Einstein frame.

When the scalar charge $\sigma \neq 0$, the conformal image of the FJNWBW geometry of GR is the Jordan frame solution

$$
\begin{gathered}
d s^{2}=-\mathrm{e}^{(\alpha+\beta) / r} d t^{2}+\mathrm{e}^{(\beta-\alpha) / r}\left(\frac{\gamma / r}{\sinh (\gamma / r)}\right)^{4} d r^{2} \\
+\mathrm{e}^{(\beta-\alpha) / r}\left(\frac{\gamma / r}{\sinh (\gamma / r)}\right)^{2} r^{2} d \Omega_{(2)}^{2} \\
\phi(r)=\phi_{0} \mathrm{e}^{-\beta / r}, \quad \beta=\frac{\sigma}{\sqrt{|2 \omega+3|}},
\end{gathered}
$$

\footnotetext{
${ }^{26} \mathrm{~A}$ casual remark to this regard is found in [392].
} 
when $\gamma \neq 0$ (the special case $\gamma=0$ is discussed later).

There is another form of the solution for imaginary $\gamma$, for which the hyperbolic sine in Eq. (175) is replaced by a sine [200]. This possibility (called a "cold black hole" in $[393,394]$ ) occurs for $2 \omega+3<0$ and makes the Einstein frame $\tilde{\phi}$ purely imaginary, giving the wrong sign to its kinetic energy density in what can be regarded as the ghost counterpart of the FJNBW solution (42), (43) discussed by Bergmann and Leipnik [167]. In this case, $\sigma$ becomes imaginary and the Wyman relation (44) between parameters becomes $-4 \gamma^{2}=\alpha^{2}-2 \sigma^{2}$ [200], where the negative signs can be absorbed making both $\sigma$ and $\gamma$ imaginary. This anomalous general solution is

$$
\begin{aligned}
& d s^{2}=-\mathrm{e}^{\frac{\alpha+\beta}{r}} d t^{2} \\
& +\mathrm{e}^{\frac{\beta-\alpha}{r}}\left(\frac{\gamma / r}{\sin (\gamma / r)}\right)^{2}\left[\left(\frac{\gamma / r}{\sin (\gamma / r)}\right)^{2} d r^{2}+r^{2} d \Omega_{(2)}^{2}\right] .
\end{aligned}
$$

Its special cases $\alpha=\beta, \alpha=(2 \omega+3) \beta$, and $\alpha=-(\omega+1) \beta$ were reported by Van den Bergh [395]. A later and more complete analysis [393] in the wider Bergmann-Wagoner class of scalar-tensor gravities with $\omega=\omega(\phi)$ found that black hole geometries ("cold black holes") can occur in these anomalous solutions. We will not discuss this ghost solution further, focusing on (175), (176) instead.

Next, one wonders about the relation of (175), (176) with the previous Campanelli-Lousto (and other) solutions. To elucidate this question when $\gamma \neq 0$, one performs the two consecutive coordinate changes

$$
\mathrm{e}^{\gamma / r}=\frac{1+B / \rho}{1-B / \rho}, \quad \bar{r}=\rho\left(1+\frac{B}{\rho}\right)^{2},
$$

introduces $\eta=2 B=\sqrt{m^{2}+\sigma^{2}}, m / \eta=-\alpha /(2 \gamma), \sigma / \eta=\beta \sqrt{|2 \omega+3|} /(2 \gamma)$, and rescales the time coordinate by $|\gamma /(2 B)|$, which recasts the pair (175), 
(176) as

$$
\begin{aligned}
d s^{2}= & -\left(1-\frac{2 \eta}{\bar{r}}\right)^{\frac{1}{\eta}\left(m-\frac{\sigma}{\sqrt{|2 \omega+3|}}\right)} d t^{2}+\left(1-\frac{2 \eta}{\bar{r}}\right)^{\frac{-1}{\eta}\left(m+\frac{\sigma}{\sqrt{|2 \omega+3|}}\right)} d \bar{r}^{2} \\
& +\left(1-\frac{2 \eta}{\bar{r}}\right)^{1-\frac{1}{\eta}\left(m+\frac{\sigma}{\sqrt{|2 \omega+3|}}\right)} \bar{r}^{2} d \Omega_{(2)}^{2} \\
\phi= & \phi_{0}\left(1-\frac{2 \eta}{\bar{r}}\right)^{\frac{\sigma}{\eta \sqrt{|2 \omega+3|}}}
\end{aligned}
$$

This is exactly the form of the Campanelli-Lousto solution with parameters ${ }^{27}$

$$
\begin{aligned}
& a_{0}=-1+\frac{1}{\eta}\left(m+\frac{\sigma}{\sqrt{|2 \omega+3|}}\right), \\
& b_{0}=-1+\frac{1}{\eta}\left(m-\frac{\sigma}{\sqrt{|2 \omega+3|}}\right) .
\end{aligned}
$$

The Campanelli-Lousto metric (172) [389, 390] is just another form of the general solution, but it is restricted by the validity of the new coordinates (and to $2 \omega+3>0$ ). Other solutions appearing in the literature, for example the Brans Class I-IV solutions ${ }^{28}$ [396] fall in the general form $(175$, (176). All this is consistent with independent realizations [399, 392, 400, 391, 398] that Campanelli-Lousto and Brans solutions describe wormhole throats or naked singularities, but not black holes.

In order to assess the physical nature of the solutions, one classifies the possible roots of $\nabla^{c} R \nabla_{c} R=0[73,71,47]$ where

$$
R(r)=\gamma \frac{\mathrm{e}^{\frac{\beta-\alpha}{2 r}}}{\sinh (\gamma / r)}
$$

is the areal radius. We have

$$
\nabla^{c} R \nabla_{c} R=g^{r r}\left(\frac{d R}{d r}\right)^{2}=\sinh ^{2}(\gamma / r)\left[\frac{\alpha-\beta}{2 \gamma}+\operatorname{coth}(\gamma / r)\right]^{2}=0 .
$$

\footnotetext{
${ }^{27}$ The anomalous case $2 \omega+3<0$ cannot be obtained here because $\gamma$ is imaginary and the new coordinates (178) are not real.

${ }^{28}$ Originally, Brans [396] reported four classes of static spherical solutions, but later on it was found that pairs of these classes are related by dualities [392, 397, 398].
} 
Real roots

$$
r_{\mathrm{H}}=\frac{2 \gamma}{\ln \left(\frac{\beta-\alpha+2 \gamma}{\beta-\alpha-2 \gamma}\right)}=\frac{\gamma}{\tanh ^{-1}\left(\frac{2 \gamma}{\beta-\alpha}\right)},
$$

exist if $(\beta-\alpha) / \gamma>0$, and are always double roots corresponding to wormhole throats. If $(\beta-\alpha) / \gamma<0$, instead, there is a naked singularity at $R=0$, highlighted by the divergence of the Ricci scalar

$$
\mathcal{R}=\frac{\omega}{\phi^{2}} \nabla^{c} \phi \nabla_{c} \phi=\left\{\begin{array}{cl}
\frac{\omega \beta^{2}}{\gamma^{4}} \mathrm{e}^{(\alpha-\beta) / r} \sinh ^{4}(\gamma / r) & \text { if } \gamma \neq 0, \\
\frac{\omega \beta^{2}}{r^{4}} \mathrm{e}^{(\alpha-\beta) / r} & \text { if } \gamma=0 .
\end{array}\right.
$$

In fact, if $\gamma \neq 0$ then when $r \rightarrow 0$ we have

$$
\mathcal{R}=\frac{\omega \beta^{2}}{16 \gamma^{4}} \mathrm{e}^{(\alpha-\beta \pm 4 \gamma) / r},
$$

where the upper [lower] sign applies if $\gamma>0[\gamma<0]$. $\mathcal{R}$ diverges as $r \rightarrow 0$ only for $\beta-\alpha<4 \gamma$ or for $\alpha-\beta>4 \gamma$, respectively.

Let us discuss now the case $\gamma=0$ : the Einstein frame FJNWBW metric degenerates into the Yilmaz geometry [179], while its Jordan frame image (175), (176) becomes the Brans Class IV solution

$$
\begin{aligned}
d s^{2} & =-\mathrm{e}^{-2 B / r} d t^{2}+\mathrm{e}^{2 B(C+1) / r}\left(d r^{2}+r^{2} d \Omega_{(2)}^{2}\right), \\
\phi & =\phi_{0} \mathrm{e}^{-B C / r},
\end{aligned}
$$

found by Brans [396] immediately after the introduction of Brans-Dicke theory, and where $B=-(\alpha+\beta) / 2, C=-2 \beta /(\alpha+\beta)$. The apparent

horizons are now located by the roots of $\left(1-\frac{\beta-\alpha}{2 r}\right)^{2}=0$. There is a double root $r_{\mathrm{H}}=(\beta-\alpha) / 2$ (and, therefore, a wormhole throat) if $\beta>\alpha$ and a naked central singularity otherwise, which matches the results of studies of the specific Brans IV class [401, 398].

The most general electrovacuum solution of the generalized scalar-tensor theory which is static, spherical, and asymptotically flat was found by Bronnikov et al. [393].

\section{A selection of dynamical solutions of Brans-Dicke theory}

In the following we review analytic and spherically symmetric solutions of scalar-tensor theories. These solutions are not general solutions in any 
sense. Usually, their stability with respect to perturbations has not been studied and they are probably fine-tuned. Their physical significance is often questionable, especially considering that many of them describe naked singularities or wormhole throats, nevertheless they provide some insight on the physical properties of these theories and are useful as examples, the catalogue of which is rather meagre.

\subsection{Clifton-Mota-Barrow spacetimes}

A family of solutions of the Brans-Dicke field equations with a perfect fluid as the matter source was found by Clifton et al. [345]. This fluid has energy density $\rho^{(\mathrm{m})}$, pressure $P^{(\mathrm{m})}$, and constant barotropic equation of state $P^{(\mathrm{m})}=(\gamma-1) \rho^{(\mathrm{m})}$. The line element and Brans-Dicke scalar are spherically symmetric and dynamical [345]

$$
d s^{2}=-e^{\nu(\bar{r})} d t^{2}+a^{2}(t) e^{\mu(\bar{r})}\left(d \bar{r}^{2}+\bar{r}^{2} d \Omega_{(2)}^{2}\right),
$$

where

$$
\begin{aligned}
e^{\nu(\bar{r})} & =\left(\frac{1-\frac{m}{2 \alpha \bar{r}}}{1+\frac{m}{2 \alpha \bar{r}}}\right)^{2 \alpha} \equiv A^{2 \alpha}, \\
e^{\mu(\bar{r})} & =\left(1+\frac{m}{2 \alpha \bar{r}}\right)^{4} A^{\frac{2}{\alpha}(\alpha-1)(\alpha+2)}, \\
a(t) & =a_{0}\left(\frac{t}{t_{0}}\right)^{\frac{2 \omega(2-\gamma)+2}{3 \omega \gamma(2-\gamma)+4}} \equiv a_{*} t^{\beta}, \\
\phi(t, \bar{r}) & =\phi_{0}\left(\frac{t}{t_{0}}\right)^{\frac{2(4-3 \gamma)}{3 \omega \gamma(2-\gamma)+4}} A^{-\frac{2}{\alpha}\left(\alpha^{2}-1\right)}, \\
\alpha & =\sqrt{\frac{2(\omega+2)}{2 \omega+3}}, \\
\rho^{(\mathrm{m})}(t, \bar{r}) & =\rho_{0}^{(\mathrm{m})}\left(\frac{a_{0}}{a(t)}\right)^{3 \gamma} A^{-2 \alpha} .
\end{aligned}
$$

$m$ is a mass parameter, while $\alpha, \phi_{0}, a_{0}, \rho_{0}^{(\mathrm{m})}$ and $t_{0}$ are positive constants ( $\alpha>0$ requires $\omega<-2$ or $\omega>-3 / 2$ ), and $\bar{r}$ is the isotropic radius (the Schwarzschild or curvature radial coordinate $r$ is given by $\left.r \equiv \bar{r}\left(1+\frac{m}{2 \alpha \bar{r}}\right)^{2}\right)$. We assume that $\omega>-3 / 2$ and $\beta \geq 0$. The line element (190) reduces to spatially flat FLRW if $m=0$. If $\gamma \neq 2$, then $\omega=(\gamma-2)^{-1}$ gives $\beta=0$ and 
the spacetime becomes static with a time-dependent scalar field. If instead $\gamma=2,4 / 3$, then $\beta=1 / 2$ and $a(t) \sim \sqrt{t}$ regardless of the value of $\omega$.

The solution can be rewritten in terms of the areal radius $R=A^{\frac{1}{\alpha}(\alpha-1)(\alpha+2)}$, obtaining $[402,47]$

$$
d s^{2}=-A^{2 \alpha} D F^{2} d \vec{t}^{2}+\left(\frac{H^{2}}{B^{4} D} R^{2} A^{2(2-\alpha)}+\frac{A^{2}}{B^{2}}\right) d R^{2}+R^{2} d \Omega_{(2)}^{2},
$$

where $H \equiv \dot{a}(t) / a(t), F$ is an integrating factor,

$$
\begin{gathered}
B(\bar{r}) \equiv A^{2}(\bar{r})+\frac{(\alpha-1)(\alpha+2)}{\alpha^{2}} \frac{m}{r}>0, \\
\psi=\frac{\dot{a}(t) r}{B^{2}} \frac{A^{\frac{-\alpha^{2}+3 \alpha-2}{\alpha}}}{D(t, \bar{r})},
\end{gathered}
$$

and

$$
D(t, \bar{r}) \equiv 1-\frac{\dot{a}^{2}(t) r^{2}}{B^{2}} A^{\frac{4}{\alpha}(\alpha-1)}
$$

The apparent horizons which (when they exist) are the positive roots of $g^{R R}=0$ have been identified in [402] for various values of the parameters. A rich variety of phenomenologies is obtained, including appearing and disappearing pairs of apparent horizons, no horizons, single horizons, and collapsing horizons. When $m=0$ there is, of course, a single FLRW apparent horizon of areal radius $R=H^{-1}$, and this horizon is found also as $\bar{r} \rightarrow+\infty$.

The static case $\beta=0$ is obtained for $\omega=(\gamma-2)^{-1}$ with $\gamma \neq 2$ (with $\omega<-2$ or $\omega>-3 / 2$ implying $\gamma>3 / 2$ or $\gamma<4 / 3$ when $\beta=0$ ). This static spacetime always contains a naked central singularity [402].

The limit to GR $\omega \rightarrow \infty$ when $\gamma \neq 0,2$ produces $\alpha \rightarrow 1, \phi \rightarrow \phi_{0}$, and the spacetime

$$
\begin{aligned}
d s^{2} & =-\left(\frac{1-\frac{m}{2 \bar{r}}}{1+\frac{m}{2 \bar{r}}}\right)^{2} d t^{2}+a^{2}(t)\left(1+\frac{m}{2 \bar{r}}\right)^{4}\left(d \bar{r}^{2}+\bar{r}^{2} d \Omega_{(2)}^{2}\right), \\
a(t) & =a_{0}\left(\frac{t}{t_{0}}\right)^{\frac{2}{3 \gamma}}, \\
\rho^{(\mathrm{m})}(t) & =\rho_{0}^{(\mathrm{m})}\left(\frac{t_{0}}{t}\right)^{2} A^{-2} .
\end{aligned}
$$


which is the generalized McVittie metric in isotropic coordinates. The mass function $M(t) \equiv m a(t) \geq 0$ is an arbitrary function of time and $G_{0}^{1} \neq 0$, corresponding to a radial energy flow. The non-rotating Thakurta solution of the Einstein equations with $M(t)=M_{0} a(t)$ and $M_{0}=$ const., which is a late-time attractor in the class of generalized McVittie solutions, is the GR limit of the Clifton-Mota-Barrow family of Brans-Dicke spacetimes.

For $\gamma=0$ the cosmic fluid reduces to a cosmological constant, $\beta \rightarrow \infty$ as $\omega \rightarrow \infty$ and $a(t)$ becomes a power law. If $\gamma=2$, then the $\omega \rightarrow \infty$ limit yields $\alpha \rightarrow 1, \phi \propto t^{-1}, a(t) \propto \sqrt{t}$, and $\rho^{(\mathrm{m})} \propto t^{-3} A^{-2}$.

\subsection{Conformally transformed Husain-Martinez-Nuñez spacetime}

Clifton et al. [345] generated another two-parameter family of dynamical and spherically symmetric solutions of the Brans-Dicke field equations by mapping the Husain-Martinez-Nuñez geometry of GR to the Jordan frame. The result is [345]

$$
\begin{aligned}
d s^{2}= & -A^{\alpha\left(1-\frac{1}{\sqrt{3} \beta}\right)}(r) d t^{2}+A^{-\alpha\left(1+\frac{1}{\sqrt{3} \beta}\right)}(r) t^{\frac{2(\beta-\sqrt{3})}{3 \beta-\sqrt{3}}} \\
& \times\left[d r^{2}+r^{2} A(r) d \Omega_{(2)}^{2}\right] \\
\phi(t, r)= & A^{\frac{ \pm 1}{2 \beta}}(r) t^{\frac{2}{\sqrt{3} \beta-1}},
\end{aligned}
$$

where $\omega>-3 / 2$ and

$$
A(r)=1-\frac{2 C}{r}, \quad \beta=\sqrt{2 \omega+3}, \quad \alpha= \pm \sqrt{3} / 2 .
$$

This is an inhomogeneous spacetime with a spatially flat FLRW "background" with scale factor

$$
a(t)=t^{\frac{\beta-\sqrt{3}}{3 \beta-\sqrt{3}}} .
$$

The apparent horizons and singularities of this rather involved solution are reported in $[403,47]$.

\subsection{Geometry conformal to Fonarev}

The Fonarev solution of GR, used as a seed and mapped to the Jordan frame, generates a four-parameter family of solutions of Brans-Dicke theory with a power-law potential, which are dynamical, spherically symmetric, and asymptotically FLRW. It contains as special cases two previously known 
classes of solutions and solves also the field equations of $f(\mathcal{R})=\mathcal{R}^{n}$ gravity. The Jordan frame scalar field is [404]

$$
\phi(t, r)=\phi_{0} \mathrm{e}^{\frac{4 \alpha a t}{\sqrt{|2 \omega+3|}}}\left(1-\frac{2 m}{r}\right)^{\frac{1}{\sqrt{|2 \omega+3|\left(1+4 \alpha^{2}\right)}}}
$$

and the corresponding scalar field potential is $V(\phi)=V_{0} \phi^{2 \beta}$ with

$$
\beta=1-\alpha \sqrt{|2 \omega+3|}, \quad V_{0}=\tilde{V}_{0} \phi_{0}^{2 \alpha \sqrt{|2 \omega+3|}} .
$$

In this case, the potential is physically motivated and well studied in cosmology and particle physics, including the pure mass, quartic, and many quintessence potentials $[405,406,15,407,408,23]$. The usual relation $g_{a b}=\phi^{-1} \tilde{g}_{a b}$ gives the Jordan frame line element

$$
\begin{aligned}
& d s^{2}=-A(r)^{\frac{1}{\sqrt{1+4 \alpha^{2}}}}\left(2 \alpha-\frac{1}{\sqrt{|2 \omega+3|}}\right) \mathrm{e}^{4 \alpha a t\left(2 \alpha-\frac{1}{\sqrt{|2 \omega+3|}}\right)} d t^{2} \\
& +\mathrm{e}^{2 a t\left(1-\frac{2 \alpha}{\sqrt{|2 \omega+3|}}\right)}\left[A(r)^{-\frac{1}{\sqrt{1+4 \alpha^{2}}}}\left(2 \alpha+\frac{1}{\sqrt{|2 \omega+3|}}\right) d r^{2}\right. \\
& \left.+A(r)^{1-\frac{1}{\sqrt{1+4 \alpha^{2}}}\left(2 \alpha+\frac{1}{\sqrt{|2 \omega+3|}}\right)} r^{2} d \Omega_{(2)}^{2}\right] .
\end{aligned}
$$

This is a family of solutions of the vacuum Brans-Dicke field equations parametrized by the four parameters $(\omega, m, a, \alpha)$ (the last three being parameters of this specific family). Special cases include the $V=0$ conformal counterpart of the Husain-Martinez-Nuñez solution and the general static solution in the Campanelli-Lousto form. This solution is, again, complicated and contains naked singularities or wormhole throats for most values of the parameters [404].

A special subcase of the Brans-Dicke solution conformal to Fonarev, which is static but has time-dependent scalar field, was used to generate new dynamical solutions using the symmetry (163)-(165) of the vacuum field equations [409].

\section{Nonminimally coupled scalar fields}

The action of gravity with a nonminimally coupled scalar field is

$$
S_{(\mathrm{NMC})}=\int d^{4} x \sqrt{-g}\left[\left(\frac{1}{2 \kappa}-\frac{\xi}{2} \phi^{2}\right) \mathcal{R}-\frac{1}{2} \nabla^{c} \phi \nabla_{c} \phi-V(\phi)\right]+S^{(\mathrm{m})},
$$


where $\xi$ is a dimensionless coupling constant. The value $\xi=1 / 6$ (conformal coupling) makes the physics of $\phi$ conformally invariant if $V=0$ or $V=\lambda \phi^{4}$, in which case $V$ is scale-free [50]. Varying the action (211) produces the field equations

$$
\begin{aligned}
& \left(1-\kappa \xi \phi^{2}\right) G_{a b}=\kappa\left\{\nabla_{a} \phi \nabla_{b} \phi-\frac{1}{2} g_{a b} \nabla^{c} \phi \nabla_{c} \phi\right. \\
& \left.-V g_{a b}+\xi\left[g_{a b} \square\left(\phi^{2}\right)-\nabla_{a} \nabla_{b}\left(\phi^{2}\right)\right]\right\}, \\
& \square \phi-\frac{d V}{d \phi}-\xi \mathcal{R} \phi=0 .
\end{aligned}
$$

They describe a scalar-tensor gravity; in fact, by redefining the scalar field and its potential as

$$
\begin{gathered}
\varphi=1-\kappa \xi \phi^{2}, \\
U(\varphi)=16 \pi V[\phi(\varphi)]=16 \pi V\left( \pm \sqrt{\frac{1-\varphi}{\kappa \xi}}\right),
\end{gathered}
$$

the nonminimally coupled action (211) becomes

$$
\begin{aligned}
S_{(\mathrm{NMC})}= & \int d^{4} x \frac{\sqrt{-g}}{16 \pi}\left[\varphi \mathcal{R}-\frac{\omega(\varphi)}{\varphi} \nabla^{c} \varphi \nabla_{c} \varphi-U(\varphi)\right] \\
& +S^{(\mathrm{m})},
\end{aligned}
$$

where

$$
\omega(\varphi)=\frac{\varphi}{4 \xi(1-\varphi)} .
$$

The nonminimal coupling of the scalar field to the Ricci scalar was originally introduced by Chernikov and Tagirov [410] in the context of classical radiation problems. It became widely known when Callan et al. [411] used it to renormalize a scalar with quartic self-interaction in curved space. A nonzero $\xi$ is generated by first loop corrections even if absent at the classical level $[412,413,414,415,416,417]$. At the classical level, conformal coupling is necessary to avoid causal pathologies in the propagation of $\phi$, i.e., to avoid any possibility that a massive scalar field propagates strictly along the light cone [418]. More precisely, waves propagating in a curved spacetime suffer backscattering from the background curvature and sharp propagation is the exception rather than the norm [419]. For scalar waves $\phi$, assuming the potential to consist of a mass term $V(\phi)=m^{2} \phi^{2} / 2$, the wave 
equation (213) admits a retarded Green function $G_{R}\left(x^{\prime}, x\right)$ as the solution of

$$
\left[g^{a^{\prime} b^{\prime}}\left(x^{\prime}\right) \nabla_{a^{\prime}} \nabla_{b^{\prime}}-m^{2}-\xi R\left(x^{\prime}\right)\right] G_{R}\left(x^{\prime}, x\right)=-\delta\left(x^{\prime}, x\right)
$$

with an impulsive source. Here $\delta\left(x^{\prime}, x\right)$ is the delta function on spacetime [420] with the boundary condition $G_{R}\left(x^{\prime}, x\right)=0$ if $x$ is in the future of $x^{\prime}$. Let $x$ and $x^{\prime}$ be points in a normal domain and let $\Gamma\left(x^{\prime}, x\right)$ be the square of the proper distance along the unique geodesic connecting these points. Then, it is well known that $G_{R}\left(x^{\prime}, x\right)$ decomposes as [421]

$$
G_{R}\left(x^{\prime}, x\right)=\Sigma\left(x^{\prime}, x\right) \delta_{R}\left(\Gamma\left(x^{\prime}, x\right)\right)+V\left(x^{\prime}, x\right) \Theta_{R}\left(-\Gamma\left(x^{\prime}, x\right)\right),
$$

where $\delta_{R}$ and $\Theta_{R}$ are the Dirac delta distribution and the Heaviside step function with support in the past of $x^{\prime}$. The coefficients $\Sigma$ and $V$ are functions of the spacetime position specified uniquely in a given geometry [420, 421]. Taylor-expanding around $x$ gives [418]

$$
\begin{aligned}
\Sigma\left(x^{\prime}, x\right) & =\frac{1}{4 \pi}+\ldots \\
V\left(x^{\prime}, x\right) & =-\frac{1}{8 \pi}\left[m^{2}+\left(\xi-\frac{1}{6}\right) R(x)\right]+\ldots
\end{aligned}
$$

Locally, the propagation of $\phi$-waves must be as in Minkowski space: if $m=0$, there should be no tails and, since in general $R(x) \neq 0$, this property is guaranteed only if $\xi=1 / 6$. If $\xi \neq 1 / 6$ there is the very disturbing possibility that a massive field propagate exactly on the light cone where $m^{2}+\left(\xi-\frac{1}{6}\right) R(x)=0$ (i.e., where the tail due to the field mass cancels exactly the tail due to backscattering off the background spacetime curvature). Indeed, one can even tailor constant curvature spaces where this happens at every spacetime point [422]. The only way to prevent this unphysical feature once and for all is by requiring that $\xi=1 / 6$.

It has been argued that nonminimal coupling is necessarily present at high curvatures $[415,417]$, that the approach to a classical universe in quantum cosmology requires $\xi \neq 0$ [423], and that nonminimal coupling could solve potential problems of primordial nucleosynthesis [424]. Nonminimal coupling has been used extensively in cosmology [e.g., 425, 426, 427, 428, 429]. Various arguments are used to advocate for different values of the coupling constant $\xi[430,431,432,433,434,435,436,437,438,439,429,440]$. 
The map to the Einstein conformal frame ${ }^{29}$ assumes the form

$$
\begin{aligned}
g_{a b} & \rightarrow \tilde{g}_{a b}=\Omega^{2} g_{a b}, \quad \Omega=\sqrt{1-\kappa \xi \phi^{2}} \\
d \tilde{\phi} & =\frac{\sqrt{1-\kappa \xi(1-6 \xi) \phi^{2}}}{1-\kappa \xi \phi^{2}} d \phi .
\end{aligned}
$$

The integration of Eq. (223) gives

$$
\tilde{\phi}=\sqrt{\frac{3}{2 \kappa}} \ln \left[\frac{\xi \sqrt{6 \kappa \phi^{2}}+\sqrt{1-\xi(1-6 \xi) \kappa \phi^{2}}}{\xi \sqrt{6 \kappa \phi^{2}}-\sqrt{1-\xi(1-6 \xi) \kappa \phi^{2}}}\right]+f(\phi),
$$

where

$$
f(\phi)=\left(\frac{1-6 \xi}{\kappa \xi}\right)^{1 / 2} \arcsin \left(\sqrt{\xi(1-6 \xi) \kappa \phi^{2}}\right)
$$

for $0<\xi<1 / 6$ and

$$
f(\phi)=\left(\frac{6 \xi-1}{\kappa \xi}\right)^{1 / 2} \operatorname{arcsinh}\left(\sqrt{\xi(6 \xi-1) \kappa \phi^{2}}\right)
$$

for $\xi>1 / 6$. When $\xi=1 / 6$, we have

$$
\tilde{\phi}=\sqrt{\frac{3}{2 \kappa}} \ln \left(\frac{\sqrt{6 / \kappa}+\phi}{\sqrt{6 / \kappa}-\phi}\right) \quad \text { if }|\phi|<\sqrt{\frac{6}{\kappa}},
$$

or

$$
\tilde{\phi}=\sqrt{\frac{3}{2 \kappa}} \ln \left(\frac{\phi-\sqrt{6 / \kappa}}{\phi+\sqrt{6 / \kappa}}\right) \quad \text { if }|\phi|>\sqrt{\frac{6}{\kappa}} .
$$

The Einstein frame scalar $\tilde{\phi}$ is minimally coupled and satisfies

$$
\tilde{\square} \tilde{\phi}-\frac{d \tilde{V}}{d \tilde{\phi}}=0
$$

where

$$
\tilde{V}(\tilde{\phi})=\frac{V[\phi(\tilde{\phi})]}{\left(1-\kappa \xi \phi^{2}\right)^{2}}
$$

with $\phi=\phi(\tilde{\phi})$ as in Eq. (224).

\footnotetext{
${ }^{29}$ Often referred to as Bekenstein transformation.
} 


\subsection{Conformal coupling}

The value $1 / 6$ of the nonminimal coupling constant $\xi$ is probably the most well studied, also in terms of exact solutions of the field equations.

\subsubsection{BBMB solution}

The Bocharova-Bronnikov-Melnikov-Bekenstein (BBMB) solution for gravity, the conformally coupled scalar, and the Maxwell field was found early on by Bocharova et al. [441] and rediscovered by Bekenstein [442]. ${ }^{30}$ The BBMB solution written in terms of the areal radius is [442]

$$
\begin{aligned}
d s^{2} & =-\left(1-\frac{m}{r}\right)^{2} d t^{2}+\frac{d r^{2}}{(1-m / r)^{2}}+r^{2} d \Omega_{(2)}^{2}, \\
\phi(r) & =\sqrt{\frac{6}{\kappa}} \frac{m}{r-m}, \\
F_{a b} & =\frac{Q}{r^{2}}\left(\delta_{a}^{1} \delta_{b}^{0}-\delta_{a}^{0} \delta_{b}^{1}\right),
\end{aligned}
$$

where $m$ and $Q$ are the mass and electric charge and $F_{a b}$ is the Maxwell tensor. The geometry is that of an extremal Reissner-Nordström black hole, but the scalar field $\phi$ is singular on the horizon $r=m$. This property is unphysical, as originally remarked by Bekenstein himself [442] and contrary to what later argued in [444], but reconsidered in $[445,446,136] .{ }^{31}$ It has also been argued that, in advanced Eddington-Finkelstein coordinates extending beyond the horizon, the BBMB geometry fails to satisfy the field equations at the horizon $[136,449]$. The usual black hole thermodynamics becomes impossible [450]. Moreover, the BBMB solution is unstable to linear perturbations [451, 452].

Following the derivations of Bocharova et al. [441] and Bekenstein [442], Xanthopoulos and Zannias [445], and later Klimcík [448], proved explicitly that the BBMB construct is the unique solution of the Einstein-conformal scalar field equations which is static, spherical, asymptotically flat, and does not have constant $\phi$. A new proof of the uniqueness of the BBMB solution

\footnotetext{
${ }^{30}$ It was rediscovered again by Fr $\varnothing y$ land [443] by integrating directly the Jordan frame field equations instead of using the conformal mapping trick (a similar, but rather confused, attempt was made in Ref. [173]).

${ }^{31}$ Curiously, in higher dimension the (unique) analogue of the BBMB solution describes a naked singularity [447, 446, 448, 219].
} 
outside the photon surface (the surface composed of the unstable circular photon orbits) was given in Tomikawa et al. [453, 454]. This proof shows also that no static multiple disconnected photon surfaces exist, and it does not assume the existence and connectedness of the photon sphere.

Rotating extensions of the BBMB black hole are derived by Astorino [455] (a previous attempt to build a slowly rotating BBMB solution in [456] failed near the horizon because it was subject to the excessively restrictive assumption of separability for the $g_{t \varphi}$ metric coefficient). The BBMB solution has also been generalized by including a cosmological constant plus a quartic potential $V(\phi)=\lambda \phi^{4}$, a Maxwell field, and different horizon topologies $[457,458,459]$. Adding a cosmological constant moves the horizon outward and the scalar field singularity remains hidden within it [457], making thermodynamics meaningful again [460]. An accelerating BBMB black hole has also been found by Charmousis et al. [461]. The Plebański-Demiański family of solutions of the Einstein equations (the most general Petrov type D solution of the Einstein-Maxwell equations $[462,463]$, which describes an accelerating and rotating black hole), generalized to include a scalar field [464], contains the BBMB geometry and its accelerated version of Charmousis et al. [461] as special cases. Since the geometry is that of an extremal ReissnerNordström black hole, the Penrose-Carter diagram is well known (see, e.g., Ref. [465].

The field equations for trace-free matter have been studied recently by Carranza et al. [466]. The solution of the coupled equations for gravity, a conformally coupled scalar field, and the source-free Maxwell field or a radiation fluid is again the BBMB geometry (233) of the extreme ReissnerNordström black hole already discussed.

The BBMB geometry is also a solution of a conformally invariant gravity theory, in which different conformal frames are pure gauge choices and the singularity of the conformal scalar field at the horizon is automatically resolved by changing gauge [467].

\subsubsection{Abreu et al. generalization to scalar field potential}

Bekenstein's solution-generating technique for a free conformally coupled scalar field [442] was used by Abreu et al. [207] for a scalar subject to a nonzero potential $V(\phi)$. Although their interest was in FLRW cosmology, the technique can be used for inhomogenous spherical spacetimes. Starting from a solution of the minimally coupled $(\xi=0)$ Einstein-Klein-Gordon equations $\left(\tilde{g}_{a b}, \tilde{\phi}, U(\tilde{\phi})\right)$ as a seed, one generates a solution of the conformally coupled 


$$
\begin{aligned}
& (\xi=1 / 6) \text { equations }\left(g_{a b}, \phi, V(\phi)\right), \text { where } \\
& g_{a b}=\Omega^{-1} \tilde{g}_{a b}, \quad \Omega^{-1}=\cosh (\zeta \tilde{\phi}), \\
& \phi=\zeta^{-1} \tanh (\zeta \tilde{\phi}), \\
& V(\phi)=\left(1-\zeta^{2} \phi^{2}\right) U\left[\frac{1}{2 \zeta} \ln \left(\frac{1+\zeta \phi}{1-\zeta \phi}\right)\right],
\end{aligned}
$$

and $\zeta=\sqrt{\kappa / 6}[207]$.

\subsection{Other time-dependent solutions}

Other time-dependent solutions of scalar-tensor gravity that we cannot analyze here for lack of space include those of Shaw and Barrow [468, 469], and Banijamali et al. [208]. A common generating technique for time dependent Einstein-scalar solutions takes known seed solutions with minimally coupled massless scalar fields and, using the conformal map to the Jordan frame, produces solutions with a nonminimally coupled field and with a perfect fluid interpretation (e.g., [470, 205, 208, 409, 471]).

\section{2. $f(\mathcal{R})$ gravity}

Quantum corrections to the Lagrangian density of GR introduce quadratic terms in the curvature. The simplest such scenario is Starobinsky's model of inflation in $f(\mathcal{R})=\mathcal{R}+\alpha \mathcal{R}^{2}$ gravity [6]. This was the first inflationary scenario proposed and is currently the one favoured by cosmological observations. Since 2003, there has been a resurgence of interest in $f(\mathcal{R})$ gravity as a way to explain the present acceleration of the universe without invoking an ad hoc dark energy [24, 25] (see [26, 27, 28] for reviews). The idea is to modify gravity at large scales and to model the universe in the context of $f(\mathcal{R})$ theories which incorporate an effective time-dependent cosmological "constant". This approach has the advantage of bypassing the extreme finetuning associated with the standard cosmological constant $\Lambda$ of the $\Lambda \mathrm{CDM}$ model (but does not solve the cosmological constant problem, of course).

Metric $f(\mathcal{R})$ gravity is nothing but an $\omega=0$ Brans-Dicke theory with a potential in disguise. The action is $[26,27,28]$

$$
S=\int d^{4} x \frac{\sqrt{-g}}{16 \pi} f(\mathcal{R})+S^{(\mathrm{m})}
$$


where $f(\mathcal{R})$ is a non-linear function of the Ricci scalar $\mathcal{R}$ and $S^{(\mathrm{m})}$ is the matter action. The field equations are the fourth order ones

$$
f^{\prime}(\mathcal{R}) \mathcal{R}_{a b}-\frac{f(\mathcal{R})}{2} g_{a b}=\nabla_{a} \nabla_{b} f^{\prime}(\mathcal{R})-g_{a b} \square f^{\prime}(\mathcal{R})+8 \pi T_{a b}^{(\mathrm{m})} .
$$

If one uses the new scalar field $\phi \equiv f^{\prime}(\mathcal{R})$ with potential

$$
V(\phi)=\phi \mathcal{R}(\phi)-\left.f(\mathcal{R}(\phi))\right|_{\phi=f^{\prime}(\mathcal{R})},
$$

one can show $[26,27,28]$ that the action (237) is equivalent to

$$
S=\int d^{4} x \frac{\sqrt{-g}}{16 \pi}[\phi \mathcal{R}-V(\phi)]+S^{(\mathrm{m})},
$$

which is a Brans-Dicke action with Brans-Dicke parameter $\omega=0$ and the potential (239).

Since the main motivation for $f(\mathcal{R})$ gravity comes from cosmology, the most well-studied analytic solutions of these theories should describe central objects embedded in FLRW universes. Very often the locally static KSdS geometry is a solution, but it is not the only one.

\subsection{Black holes and no-hair theorems in $\boldsymbol{f}(\mathcal{R})$ gravity}

If a metric $f(\mathcal{R})$ theory of gravity admits an electrovacuum solution with $\mathcal{R}=0$ (i.e., if $f(0)=0$ ), the no-hair theorems of scalar-tensor gravity apply in vacuo. Strictly speaking, such a theory misses the motivation of explaining the present acceleration of the universe without dark energy, which motivated the resurgence of $f(\mathcal{R})$ theories. In these theories and also in theories with $f(0) \neq 0$, to the extent that the cosmological asymptotics can be neglected, the usual scalar-tensor no-hair theorems apply and then the only physical black holes which are stationary and stable are the Kerr ones of GR. The argument relies on the equivalence between metric $f(\mathcal{R})$ gravity and an $\omega=0$ Brans-Dicke theory with a complicated potential. Furthermore, ${ }^{32}$ it is performed in the Einstein conformal frame. An alternative proof was obtained by Cañate [473] by analyzing directly the fourth order electrovacuum field equations of $f(\mathcal{R})$ gravity (with $f$ a function continuous with its first and second derivatives and satisfying $f(0)=0$ ) without resorting to the equivalence with Brans-Dicke theory. The proof mirrors the standard one in scalar-tensor gravity using integrals, and does not apply to

\footnotetext{
${ }^{32}$ With the exception of Ref. [472]
} 
the special theory $f(\mathcal{R})=\mathcal{R}^{2} .{ }^{33}$ This purely quadratic theory is pathological since it exhibits a restricted conformal invariance and does not admit a Newtonian limit [475], however it is a good approximation to the Starobinsky [6] inflationary scenario based on $f(\mathcal{R})=\mathcal{R}+\alpha \mathcal{R}^{2}$ in the early universe. The special case $f(\mathcal{R})=\mathcal{R}^{2}$ was covered separately by Sultana and Kazanas [476] (their proof uses the equivalence with $\omega=0$ Brans-Dicke theory and generalizes the Jordan frame proof ${ }^{34}$ of Faraoni [472] for vanishing potential to the case of a quadratic potential, which is well-known to disappear from the field equation for the Brans-Dicke scalar). More important, the proof of Cañate [473] is extended to asymptotically de Sitter electrovacuum stationary black holes in theories which admit constant curvature solutions $\mathcal{R}=$ const. $>0$.

Separate no-hair theorems restricted to specific $f(\mathcal{R})$ theories were given earlier, including the Starobinsky model $f(\mathcal{R})=\mathcal{R}+\alpha \mathcal{R}^{2}[478]$ and $f(\mathcal{R})=$ $\mathcal{R}^{n}[479,480,481,482,483]$.

\subsection{Clifton-Barrow static solution of $\boldsymbol{f}(\mathcal{R})=\mathcal{R}^{1+\delta}$ gravity}

This solution of $f(\mathcal{R})=\mathcal{R}^{1+\delta}$ gravity reads [484, 481]

$$
d s^{2}=-A_{1}(r) d t^{2}+\frac{d r^{2}}{B_{1}(r)}+r^{2} d \Omega_{(2)}^{2},
$$

where

$$
\begin{aligned}
& A_{1}(r)=r^{\frac{2 \delta(1+2 \delta)}{1-\delta}}+\frac{C_{1}}{r^{\frac{1-4 \delta}{1-\delta}}} \\
& B_{1}(r)=\frac{(1-\delta)^{2}}{\left(1-2 \delta+4 \delta^{2}\right)[1-2 \delta(1+\delta)]}\left(1+\frac{C_{1}}{r^{\frac{1-2 \delta+4 \delta^{2}}{1-\delta}}}\right) .
\end{aligned}
$$

This static geometry is conformal to the special case of a solution of EinsteinMaxwell-dilaton gravity found by Chan et al. [485] obtained when the electric charge vanishes [481]. It reduces to Schwarzschild when $\delta \rightarrow 0$. It has been shown to describe a black hole by Cognola et al. [486].

\footnotetext{
${ }^{33}$ Care must be taken, where the map between conformal frames breaks down (in $\mathcal{R}^{2}$ gravity, where $\mathcal{R}=0[474]$ ).

${ }^{34}$ This proof is repeated, but specifically for $f(\mathcal{R})$ gravity instead of general scalar-tensor theory, in [477].
} 


\subsection{Clifton's inhomogeneous cosmology in $\mathcal{R}^{1+\boldsymbol{\delta}}$ gravity}

A spherically symmetric and dynamical solution of vacuum $f(\mathcal{R})=\mathcal{R}^{1+\delta}$ gravity is due to Clifton [481]. Solar System tests of gravity constrain the parameter $\delta$ to the range $\delta=(-1.1 \pm 1.2) \cdot 10^{-5}[484,480,487]$. Furthermore, it must be $f^{\prime \prime}(\mathcal{R}) \geq 0$ for stability [488], which implies $\delta \geq 0$ and we take the mass parameter $C_{2}>0$.

The line element of the Clifton solution in isotropic coordinates reads [481]

$$
d s^{2}=-A_{2}(\bar{r}) d t^{2}+a^{2}(t) B_{2}(\bar{r})\left(d \bar{r}^{2}+\bar{r}^{2} d \Omega_{(2)}^{2}\right)
$$

where

$$
\begin{aligned}
A_{2}(\bar{r}) & =\left(\frac{1-C_{2} / \bar{r}}{1+C_{2} / \bar{r}}\right)^{2 / q}, \\
B_{2}(\bar{r}) & =\left(1+\frac{C_{2}}{\bar{r}}\right)^{4} A_{2}(\bar{r})^{q+2 \delta-1}, \\
a(t) & =t^{\frac{\delta(1+2 \delta)}{1-\delta}} \\
q^{2} & =1-2 \delta+4 \delta^{2} .
\end{aligned}
$$

It reduces to the FLRW line element when $C_{2}=0$. In the GR limit $\delta \rightarrow 0$, the geometry (244) reduces to the Schwarzschild one. The Clifton spacetime (244)-(248) is conformal to the Fonarev solution of GR which is conformally static [220], therefore it is also conformally static (they are both conformal to the FJNWBW geometry). The presence of a central spacetime singularity and of dynamical apparent horizons have been investigated in [489]. At least for some values of the parameters $C_{2}$ and $\delta$, the phenomenology of apparent horizons involves a cosmological horizon accompanied by the creation of a pair of apparent horizons, which then merge and disappear [489]. This apparent horizon phenomenology is qualitatively the same as that of the Husain-Martine-Nuñez solution of the Einstein equations [210].

\subsection{Conformal image of the Fonarev solution}

By setting $\omega=0$ in the Brans-Dicke analogue of the Fonarev solution, one obtains a solution of $f(\mathcal{R})$ gravity since the power-law potential $V(\phi)$ corresponds to the effective potential

$$
V_{0}\left[f^{\prime}(\mathcal{R})\right]^{2 \beta}=\mathcal{R} f^{\prime}(\mathcal{R})-f(\mathcal{R}), \quad \beta=1-\alpha \sqrt{3} .
$$


for $f(\mathcal{R})=\mu \mathcal{R}^{n}$ and

$$
\begin{aligned}
\beta & =\frac{n}{2(n-1)}, \\
V_{0} & =\frac{n-1}{n^{2 \beta}} \mu^{1-2 \beta},
\end{aligned}
$$

with $n \neq 1$. Then, the parameter $\alpha$ of the family of solutions (208), (210) is

$$
\alpha=\frac{n-2}{2 \sqrt{3}(n-1)} .
$$

The conformal image of the Husain-Martinez-Nuñez solution is obtained for $\alpha= \pm \sqrt{3} / 2$ and is, therefore, a solution of $f(\mathcal{R})=\mu \mathcal{R}^{n}$ gravity for $n=1 / 2,5 / 4$ (however, these values of $n$ are ruled out by Solar System experiments $[481,480,487]$ ). Any $f(\mathcal{R})$ theory must satisfy $f^{\prime}>0$ (in order for the graviton to carry positive energy) and $f^{\prime \prime} \geq 0$ (to ensure local stability) $[26,27,28]$. Then, $\delta \geq 0$,

$$
\alpha=-\frac{(1-\delta)}{2 \sqrt{3} \delta}, \quad \beta=\frac{1+\delta}{2 \delta}
$$

(with $\alpha<0$ ), and

$$
\begin{aligned}
d s^{2}= & -A(r)^{-\frac{1}{\sqrt{1-2 \delta+4 \delta^{2}}}} \mathrm{e}^{\frac{2(1-\delta) a t}{\sqrt{3} \delta}} d t^{2} \\
& +\mathrm{e}^{\frac{2(1+2 \delta) a t}{3 \delta}}\left[A(r)^{\frac{1-2 \delta}{\sqrt{1-2 \delta+4 \delta^{2}}}} d r^{2}+A(r)^{\frac{1-2 \delta}{\sqrt{1-2 \delta+4 \delta^{2}}}-1} r^{2} d \Omega_{(2)}^{2}\right] .
\end{aligned}
$$

\subsection{Other solutions}

In addition to the previous spacetimes, various other analytic and spherically symmetric solutions of various $f(\mathcal{R})$ theories of gravity have been proposed over the years. Sometimes the form of the function $f(\mathcal{R})$ is not fixed and is found together with the specific spherical solution, which means that physical motivation is lacking - indeed many forms of $f(\mathcal{R})$ are already ruled out by the existing experimental constraints and other forms are overly complicated. As a general rule, it is easier to find static solutions with constant Ricci curvature, an avenue which has been explored (see [490, 491] for a summary of approximate solutions with constant $\mathcal{R}$ ) but the resulting catalogue of physically motivated solutions is still surprisingly scarce. Due to 
space limits, we can only list references without analyzing these solutions in detail, including $[492,493,494,495,496,497,498,499,500,501,502,503$, 504, 505, 506, 507, 508].

Sometimes exact solutions can be obtained by matching interior and exterior spacetime regions through appropriate matching conditions: these matching conditions have been studied specifically for $f(\mathcal{R})$ gravity by Deruelle et al. [509], Senovilla [510], Clifton [511], Reina et al. [512], and Chakrabarti et al. [513].

\section{Horndeski gravity}

The problematic ultraviolet limit of GR and the need to include ad hoc exotic forms of matter to account for the late-time acceleration of the universe suggest another generalization of Einstein gravity. A minimal modification of GR is obtained by relaxing some of the assumptions of Lovelock's theorem $[514,515]$. This can be achieved by adding new degrees of freedom to the theory, altering the number of spacetime dimensions, including higher order derivatives of the metric in the action, or adding non-local terms to the theory. Nonetheless, most of these modifications of GR lead to effective low-energy models that merely involve a scalar degree of freedom added to the metric. This general result embodies the power of scalar-tensor theories, combining their (apparent) simplicity and minimality with the ability to capture the main features of more complex scenarios as effective models.

When formulating a classical field theory, it is necessary to avoid phenomena that would make it ill-posed from the start. An example of such issues is ghost-like instability. To this regard, a general theorem by Ostrogradsky [516] (see also [517]) states that, in classical mechanics, a non-degenerate Lagrangian containing time derivatives higher than first order leads to a linearly unstable Hamiltonian. Likewise, higher order non-degenerate classical field theories are affected by ghost-like (or Ostrogradsky) instabilities. Thus, limiting ourselves to local theories with equations of motion containing field derivatives not higher than second order constitutes a sufficient condition to avoid Ostrogradsky instabilities.

Horndeski gravity [56] is the most general scalar-tensor theory with second order equations of motion. In its modern formulation, this theory is 
expressed in terms of the generalized Galileon Lagrangian that reads

$$
\begin{aligned}
\mathcal{L}= & \mathcal{L}_{2}+\mathcal{L}_{3}+\mathcal{L}_{4}+\mathcal{L}_{5}, \\
\mathcal{L}_{2}= & G_{2}, \\
\mathcal{L}_{3}= & -G_{3} \square \phi, \\
\mathcal{L}_{4}= & G_{4} R+G_{4 X}\left[(\square \phi)^{2}-\left(\nabla_{a} \nabla_{b} \phi\right)^{2}\right], \\
\mathcal{L}_{5}= & G_{5} G_{a b} \nabla^{a} \nabla^{b} \phi-\frac{G_{5 X}}{6}\left[(\square \phi)^{3}\right. \\
& \left.-3 \square \phi\left(\nabla_{a} \nabla_{b} \phi\right)^{2}+2\left(\nabla_{a} \nabla_{b} \phi\right)^{3}\right],
\end{aligned}
$$

where the $G_{i}(\phi, X)(i=2,3,4,5)$ are arbitrary functions of the scalar field $\phi$ and of the canonical kinetic term $X \equiv-(1 / 2) \partial_{\mu} \phi \partial^{\mu} \phi$. According to standard notation, if $f$ is a function of $X$ then $f_{X} \equiv \partial f / \partial X$,

$$
\left(\nabla_{a} \nabla_{b} \phi\right)^{2} \equiv \nabla_{a} \nabla_{b} \phi \nabla^{a} \nabla^{b} \phi
$$

and

$$
\left(\nabla_{a} \nabla_{b} \phi\right)^{3} \equiv \nabla_{a} \nabla_{c} \phi \nabla^{c} \nabla^{d} \phi \nabla_{d} \nabla^{a} \phi
$$

Then, given the action

$$
S\left[g_{a b}, \phi\right]=\int d^{4} x \sqrt{-g}\left(\mathcal{L}_{2}+\mathcal{L}_{3}+\mathcal{L}_{4}+\mathcal{L}_{5}\right),
$$

we define

$$
\mathcal{E}_{\phi} \equiv \frac{1}{\sqrt{-g}} \frac{\delta S}{\delta \phi}, \quad \mathcal{E}_{a b} \equiv \frac{2}{\sqrt{-g}} \frac{\delta S}{\delta g^{a b}} .
$$

The field equations of the theory defined by the action (258) then read $\mathcal{E}_{\phi}=0$ and $\mathcal{E}_{a b}=0$.

The connection with the Galileon model is particularly instructive and offers a rather pedagogical path toward the derivation of the Horndeski Lagrangian. The Galileon theory was originally proposed by Nicolis et al. [518] as a generalization of the Dvali-Gabadadze-Porrati (DGP) [519] fourdimensional effective theory. Galileon theory represents the most general ghost-free scalar field theory on a four-dimensional Minkowski spacetime, 
which is symmetric under the field transformation $\phi(x) \rightarrow \phi(x)+b_{\mu} x^{\mu}+c$ (where $b_{\mu}$ and $c$ are constants) and leads to a second order field equation. Deffayet et al. [520] extended the Galileon model to curved space ("covariant Galileon"). For the covariant Galileon, the symmetry $\phi(x) \rightarrow \phi(x)+b_{\mu} x^{\mu}+c$ must be dropped in favor of general covariance. Kobayashi et al. [521] showed that the generalized Galileon Lagrangian (255) of Deffayet et al. [522], representing a natural extension of the covariant Galileon and preserving the second order nature of the field equations, is equivalent to Horndeski's old and forgotten theory.

One of the main motivations for Horndeski's theory is providing an alternative to dark energy for modelling the cosmic evolution. To this end, one needs to make sure that such modifications of GR, while properly accounting for the cosmic expansion, do not affect the predictions for Solar System experiments. In other words, Solar System physics should be screened from the effects of the new scalar degree of freedom $\phi$. In scalar-tensor theories, this goal can be achieved by either making $\phi$ effectively massive at short scales through the chameleon mechanism [523, 524], or else the theory itself exploits its nonlinearities to screen physics below a certain scale from the effects of $\phi$ through the Vainshtein mechanism [525] (see also the review [526] and the references therein). The latter, in particular, plays a fundamental role for cosmological models in Horndeski gravity.

Another important theoretical reason for studying more general scalartensor theories relies on potential violations of the no-hair theorems of GR. Indeed, while these theorems can be easily extended to simpler scalar-tensor theories as discussed earlier, since most of these models turn out to be singular at the black hole horizon unless the scalar field is in a trivial configuration, the situation is significantly more complicated in Horndeski gravity and its generalizations. We refer the interested reader to the excellent review by Kobayashi [527] for a discussion of the literature on this subject.

It is also important to mention that the combined detection of gravitational waves (GW170817) and of a $\gamma$-ray burst (GRB170817A) from a binary neutron star merger $[528,529]$ has put severe constraints on viable scalar-tensor models (e.g., [530]) by setting the bounds

$$
-3 \times 10^{-15}<c_{\mathrm{GW}}-1<7 \times 10^{-16}
$$

on the speed of gravitational waves $c_{\mathrm{GW}}$ ([527] and references therein).

From a field-theoretic perspective, Horndeski gravity is an effective field theory (EFT) defined in such a way that certain higher derivative operators become as relevant as lower order ones within the regime of validity of the 
low-energy model. For such an EFT to be physically consistent one has to require, within the regime of applicability of the model, that: a) the leading higher derivative terms do not introduce ghost instabilities; b) quantum corrections must be suppressed in order not to spoil the computation of physical observables. While in the case of Horndeski gravity a) is fulfilled by construction, the point b) requires the theory to exhibit some "nonrenormalization" properties. For a detailed discussion of the EFT approach to higher-derivative scalar-tensor theories we refer the interested reader to $[531,532,533,534,535,536]$ and the references therein.

We begin by reviewing some generalities of the no-hair theorems in Horndeski gravity, focusing on the case of shift-symmetric theories and on possible ways to evade them. We then discuss two important classes of non-trivial hairy solutions exploiting the loopholes in the no-hair argument of Hui and Nicolis [537]. This discussion is not meant to be an in-depth analysis of the literature on exact solutions of Horndeski gravity (a topic that would deserve to be surveyed on its own), but it is rather a summary of the results capturing the main features of hairy black hole solutions within this theory. We refer the interested reader to $[140,384,527,538]$ for a discussion of the relevant literature.

\subsection{Hair or no-hair?}

Shift-symmetric Horndeski theories are a subclass of Horndeski gravity equipped with a residual Galileon symmetry from the flat space case. Specifically, they preserve the invariance under the transformation $\phi \rightarrow \phi+c$ (with $c$ a constant) even for curved manifolds. This class is defined by the Lagrangian (255) with the condition that $G_{2}, \ldots, G_{5}$ be arbitrary functions of $X$ and do not depend explicitly on $\phi$. Clearly, this shift symmetry is associated with a covariantly conserved current $J^{a}$ (see, e.g., [384] for its explicit form).

The most stringent theorem for shift-symmetric Horndeski theories [384] states that assuming: (i) spherically symmetric spacetime with a static scalar field; (ii) asymptotic flatness, $\phi^{\prime} \equiv \partial_{r} \phi \rightarrow 0$ as $r \rightarrow \infty$, and $J^{2} \equiv J^{a} J_{a}$ finite at the horizon; (iii) there is a canonical kinetic term $X$ in the action and the $G_{i}$ are such that their $X$-derivatives contain only positive or zero powers of $X$, then the scalar field is constant and the spacetime is isometric to the Schwarzschild spacetime.

Taking a deeper look at (i-iii) one has that: 
- (i) implies that the spacetime metric and the scalar field take the form

$$
\begin{aligned}
d s^{2} & =-\mathcal{A}(r) d t^{2}+\mathcal{B}(r)^{-1} d r^{2}+R^{2}(r) d \Omega_{(2)}^{2}, \\
\phi & =\phi(r),
\end{aligned}
$$

with $R(r)$ denoting the areal radius. Hence, the norm of the current reduces to $J^{2}=\left(J^{r}\right)^{2} / \mathcal{B}(r)$.

- Since the location $r=r_{\mathrm{H}}$ of the horizon is determined by the largest root of $\mathcal{B}(r)=0$, the requirement (ii) concerning the regularity of $J^{2}$ at the horizon suggests that $J^{r}$ should vanish at $r_{\mathrm{H}}$. Furthermore, the conservation of $J^{a}$ reduces to $\left(R^{2} J^{r}\right)^{\prime}=0$ (with a prime denoting differentiation with respect to $r$ ), leading to $R^{2} J^{r}=$ constant. However, since $\left.R^{2} J^{r}\right|_{r=r_{\mathrm{H}}}=0$, then $J^{r}(r)=0, \forall r$.

- Specializing the general result for the conserved current $J^{a}$ in [384] to the symmetries of the problem, one has that $J^{r}$ takes the general form

$$
J^{r}=\mathcal{B} \phi^{\prime} F\left(\phi^{\prime}, g, g^{\prime}, g^{\prime \prime}\right)
$$

with $F$ a function of the first radial derivative of the field and of the metric and its first and second radial derivatives.

- (iii) and the requirement of asymptotic flatness then imply that $\phi^{\prime}(r)=$ $0, \forall r$, leading to a solution isometric to the Schwarzschild spacetime.

The first no-hair argument, by Hui and Nicolis [537], for black holes in Horndeski gravity relied heavily on the idea that the function $F$ in (263) would approach a non-zero constant at infinity, provided that one requires the theory to have a standard kinetic term in the weak field regime. Thus, according to Hui and Nicolis [537], asymptotic flatness implies that $\mathcal{A}, \mathcal{B} \rightarrow 1$ and $\phi^{\prime} \rightarrow 0$ at infinity. If one then moves continuously towards the horizon, $\phi^{\prime}$ can become non-zero while $\mathcal{B}$ and $F$ remain non-zero, contradicting the condition $J^{r}=0$ everywhere. This should then imply that $\phi^{\prime}(r)=0$ everywhere, exploiting a much weaker condition than (iii). However, this argument has two major loopholes allowing for hairy black hole solutions $[383,539]$ as we discuss in detail in the following sections. Furthermore, one can also find hairy solutions by relaxing some of the conditions in (i-iii).

To conclude this brief section on no-go results for black holes in shiftsymmetric Horndeski theories, we mention that black hole solutions of nonshift-symmetric models have been obtained analytically as effective models 
emerging from the Kaluza-Klein reductions of specific truncations of Lovelock gravity [540]. Hence, one can arguably consider these solutions as peculiar to Horndeski gravity and, for this reason, they will not be discussed here.

\subsection{Perturbative and numerical solutions}

We begin by recalling the action of Einstein-dilaton-Gauss-Bonnet (EdGB) gravity with linear coupling [541]

$$
S=\frac{1}{8 \pi} \int d^{4} x \sqrt{-g}\left(\frac{\mathcal{R}}{2}-\frac{1}{2} \nabla_{a} \phi \nabla^{a} \phi+\alpha \phi \mathcal{G}\right),
$$

where $\alpha$ has the dimensions of a squared length and

$$
\mathcal{G} \equiv \mathcal{R}^{2}-4 \mathcal{R}_{a b} \mathcal{R}^{a b}+\mathcal{R}_{a b c d} \mathcal{R}^{a b c d}
$$

is the Gauss-Bonnet integrand. This Lagrangian is a shift-symmetric Horndeski Lagrangian with

$$
G_{2}=X, G_{3}=0, G_{4}=\frac{1}{2}, G_{5}=-4 \alpha \ln |X| .
$$

The variation of the action (264) yields the field equations [541]

$$
\begin{gathered}
\mathcal{E}_{a b}=G_{a b}-\mathcal{T}_{a b}=0, \\
\mathcal{E}_{\phi}=\square \phi+\alpha \mathcal{G}=0,
\end{gathered}
$$

with

$$
\begin{aligned}
\mathcal{T}_{a b}= & \nabla_{a} \phi \nabla_{b} \phi-\frac{1}{2} g_{a b} \nabla_{c} \phi \nabla^{c} \phi \\
& -\alpha\left(g_{d a} g_{e b}+g_{d b} g_{e a}\right) \nabla_{f}\left(\nabla_{m} \phi \epsilon^{m e p q} \epsilon^{d f r s} \mathcal{R}_{r s p q}\right)
\end{aligned}
$$

denoting the effective stress-energy tensor, and where $\epsilon^{a b c d}$ is the Levi-Civita pseudotensor. The covariantly conserved current $J^{a}$ associated with the shift symmetry is [541]

$$
J^{a}=\sqrt{-g}\left(\nabla^{a} \phi+\alpha \tilde{\mathcal{G}}^{a}\right),
$$

where the explicit form of $\tilde{\mathcal{G}}^{a}$ is found by taking the general form of $J^{a}$ for a shift-symmetric Horndeski Lagrangian (reported in [384]) and setting the functions $G_{2}, \ldots, G_{5}$ as in (266). 
With the ansatz (261) parametrized in terms of the areal radius $R$, one finds that (270) reduces to [538]

$$
J^{R}=-\mathcal{B} \phi^{\prime}-4 \alpha \frac{\mathcal{A}^{\prime}}{\mathcal{A}} \frac{\mathcal{B}(\mathcal{B}-1)}{R^{2}},
$$

where now the prime denotes differentiation with respect to $R$. Assuming asymptotic flatness, $J^{R}$ vanishes at infinity, though now the second term allows for a non-trivial $\phi^{\prime}$ when $J^{R}=0$.

Because of the complexity of the field equations (267) and (268) even assuming (261) and asymptotic flatness, no closed form expression is known for the solution. The problem can be treated numerically and perturbatively. A perturbative solution of stringy gravity found by Campbell, Kaloper, and Olive [542] contains, as a special case, the more recent proposal of Sotiriou and Zhou [541]. A close dilatonic gravity theory with exponential potential has been solved numerically by Kanti, Mavromatos, Rizos, Tamvakis, and Winstanley [543]. The Campbell-Kaloper-Olive-Sotiriou-Zhou solution harbours a curvature singularity at a finite areal radius and can be cloaked by a horizon if the black hole is sufficiently massive [541].

\subsection{Babichev-Charmousis class of solutions}

Consider the subset of shift-symmetric Horndeski theories characterized by the Lagrangian [539]

$$
\mathcal{L}=\mathcal{R}-\eta \nabla^{c} \phi \nabla_{c} \phi+\beta G^{a b} \nabla_{a} \phi \nabla_{b} \phi-2 \Lambda,
$$

where $\eta, \beta, \Lambda$ are constants, which is obtained from (255) by setting $G_{3}=$ $G_{5}=0, G_{2}=-2 \Lambda+2 \eta X$, and $G_{4}=1+\beta X$. This Lagrangian contains an explicit non-minimal coupling between the Einstein tensor and the scalar field in $G^{a b} \nabla_{a} \phi \nabla_{b} \phi$, which corresponds to a specific realization of the "John Lagrangian" of the "Fab Four" subclass of Horndeski gravity [544]. The corresponding field equations are [384, 539]

$$
\begin{aligned}
\mathcal{E}_{a b}= & G_{a b}-\eta\left[\nabla_{a} \phi \nabla_{b} \phi-\frac{g_{a b}}{2} \nabla^{c} \phi \nabla_{c} \phi\right]+\Lambda g_{a b} \\
& +\frac{\beta}{2}\left[\left(\nabla^{c} \phi \nabla_{c} \phi\right) G_{a b}+2 P_{a c b d} \nabla^{c} \phi \nabla^{d} \phi\right. \\
& \left.+g_{a c} \delta_{b g h}^{c e f} \nabla^{g} \nabla_{e} \phi \nabla^{h} \nabla_{f} \phi\right]=0,
\end{aligned}
$$


while the equation for the scalar field can be recast in terms of the current conservation associated with the shift symmetry of the theory

$$
\nabla_{a} J^{a}=0, \quad J^{a}=\left(\eta g^{a b}-\beta G^{a b}\right) \nabla_{b} \phi .
$$

Here

and

$$
\delta_{c d f}^{a b e} \equiv 3 ! \delta_{[c}^{a} \delta_{d}^{b} \delta_{f]}^{e}
$$

$$
P_{a b c d} \equiv(-1 / 4) \epsilon_{a b e f} \mathcal{R}^{e f g h} \epsilon_{c d g h}
$$

denotes the double dual of the Riemann tensor. The form of the current $J^{a}$ offers a natural way to evade the Hui-Nicolis no-hair argument. In fact, in spherical symmetry and assuming the ansatz (261) parametrized in terms of the areal radius $R$, the condition $J^{R}=0$ is satisfied by either a constant scalar field or, more interestingly, by configurations such that $\eta g^{R R}-\beta G^{R R}=0$ allowing for a non-vanishing gradient $\nabla_{c} \phi$.

Considering the ansatz (261), again parametrized in terms of the areal radius $R$ and $\phi=\phi(t, R)$, then the only ansatz for $\phi$ compatible with Eqs. (273) and (274) is [384, 539]

$$
\phi=q t+\psi(R),
$$

with $q=$ const. This condition is evaded by self-tuning flat space solutions [384] that are not of interest here.

Setting $\eta=1 / 2$, according to the canonical normalization of the scalar field, Babichev and Charmousis [384] proved that, assuming (261) and (277), the general solution of the theory $(272)$ can be expressed in terms of the solution of the algebraic equation for $k(R)$

$$
(q \beta)^{2}\left(1+\frac{R^{2}}{2 \beta}\right)^{2}-\left(2+\frac{1-2 \beta \Lambda}{2 \beta} R^{2}\right) k(R)+C_{0} k^{3 / 2}(R)=0,
$$

with $C_{0}$ is an integration constant. ${ }^{35}$ Then, the metric components are

$$
\begin{aligned}
& \mathcal{A}(R)=-\frac{\mu}{R}+\frac{1}{\beta R} \int^{R} \frac{k(\bar{r})}{1+\left(\bar{r}^{2} / 2 \beta\right)} d \bar{r}, \\
& \mathcal{B}(R)=\left(1+\frac{R^{2}}{2 \beta}\right)^{2} \frac{\beta \mathcal{A}(R)}{k(R)},
\end{aligned}
$$

\footnotetext{
${ }^{35}$ The solution of Babichev and Charmousis [384] is a bit more general than the one discussed here since it also includes the cases of planar and hyperbolic symmetries, however here we are only interested in spherical solutions.
} 
while the radial dependence of the scalar field is given by

$$
\psi^{\prime}(R)= \pm \frac{\sqrt{R}}{\left(1+\frac{R^{2}}{2 \beta}\right) \mathcal{A}}\left[q^{2}\left(1+\frac{R^{2}}{2 \beta}\right) \mathcal{A}^{\prime}-\frac{1+2 \beta \Lambda}{4 \beta^{2}}\left(R^{2} \mathcal{A}^{2}\right)^{\prime}\right]^{1 / 2}
$$

where $\mu$ is an integration constant.

We highlight three relevant examples in this general class of solutions:

- Self-tuned Schwarzschild-de-Sitter solution [539]: If one looks for solutions with de Sitter or anti-de-Sitter asymptotics, assuming $\mathcal{A}(R)=\mathcal{B}(R)$ and taking $\beta q^{2}=1+2 \beta \Lambda$ and $C_{0}=(1-2 \beta \Lambda) / \sqrt{\beta}$ yields

$$
\begin{aligned}
\mathcal{A}(R) & =\mathcal{B}(R)=1-\frac{\mu}{R}-\frac{\Lambda_{\mathrm{eff}}}{3} R^{2}, \\
\psi^{\prime}(R) & = \pm \frac{q}{\mathcal{A}(R)} \sqrt{1-\mathcal{A}(R)},
\end{aligned}
$$

where the effective cosmological constant is $\Lambda_{\text {eff }}=-1 / 2 \beta$, which is positive if $\beta<0$. The self-tuning nature of this solution is due to the fact that the vacuum value of $\Lambda$ does not affect the geometry of the solution.

- Rinaldi and generalized Rinaldi solutions [545, 546, 547]: Without entering into the details of the derivation, these solutions correspond to $q=0$ and $\beta>0$ and are given by

$$
\begin{aligned}
\mathcal{A}(R)= & 1-\frac{\mu}{R}-\frac{\Lambda_{\mathrm{eff}}}{3} R^{2} \\
& -\frac{\left(1+2 \beta \Lambda_{\mathrm{eff}}\right)^{2}}{8 \beta \Lambda_{\mathrm{eff}}} \frac{\arctan (R / \sqrt{2 \beta})}{R / \sqrt{2 \beta}}, \\
\mathcal{B}(R)= & \frac{2 \beta+R^{2}}{2 \beta} \frac{\mathcal{A}}{(R \mathcal{A})^{\prime}}, \\
\phi^{\prime 2}= & \frac{R^{2}\left(1+2 \beta \Lambda_{\mathrm{eff}}\right)\left(1-2 \beta \Lambda_{\mathrm{eff}}-2 \Lambda_{\mathrm{eff}} R^{2}\right)^{2}}{\Lambda_{\mathrm{eff}}\left(1-2 \beta \Lambda_{\mathrm{eff}}\right)\left(2 \beta+R^{2}\right)^{3} \mathcal{A}(R)},
\end{aligned}
$$


with

$$
\Lambda_{\mathrm{eff}}=\frac{2 \beta \Lambda-1}{2 \beta(2 \beta \Lambda+3)} .
$$

The original Rinaldi solution [545] was obtained for $\Lambda=0$ and was affected by an unphysical behavior of the scalar field beyond the horizon (i.e., $\phi$ becomes imaginary inside the horizon). It was then pointed out by Anabalón et al. [546] and Minamitsuji [547] that this behavior of $\phi$ can be healed with the addition of a bare cosmological constant $\Lambda \neq 0$.

- Stealth Schwarzschild black hole [539]: Let $\Lambda=\eta=0$, then (278) is solved by $k(R)=$ constant. If one sets $k(R)=\beta$, then

$$
\mathcal{A}(R)=\mathcal{B}(R)=1-\frac{\mu}{R},
$$

and

$$
\psi^{\prime}(R)= \pm q \frac{\sqrt{\mu R}}{R-\mu} .
$$

Integrating the latter and substituting the result in (277), one finds

$$
\phi_{ \pm}(t, R)=q t \pm q \mu\left[2 \sqrt{\frac{R}{\mu}}+\ln \left(\frac{\sqrt{R}-\sqrt{\mu}}{\sqrt{R}+\sqrt{\mu}}\right)\right]+\phi_{0},
$$

with $\phi_{0}$ an integration constant.

The defining feature of this solution is that, while the geometry coincides with that of the Schwarzschild solution of GR, it is equipped with a non-trivial, time-dependent, scalar field.

\section{Beyond Horndeski: DHOST gravity}

In the spirit of keeping only one scalar field $\phi$ in the theory in addition to the GR graviton (i.e., three degrees of freedom), attempts to generalize scalar-tensor gravity beyond Horndeski theory were made by allowing for derivatives of higher order than second. As expected, these attempts usually run into the Ostrogradsky instability. However, it was discovered that certain higher derivative theories are, rather surprisingly, healthy in this respect $[57,58]$. A wider class of theories with this feature was then discovered, in which the Ostrogradsky ghost is exorcised by imposing a phase space constraint which makes the Lagrangian density degenerate 
$[548,549,550,551,552,553,554]$. These Degenerate Higher Order ScalarTensor (DHOST) theories have been the subject of much attention, especially in view of their confrontation with observations. The multi-messenger event GW170817-GRB170817 [555] and, in addition, the study of stability against perturbations $^{36}[556,557,558,559,560]$ impose severe constraints on DHOST theories (see Refs. [561, 527] for reviews).

Denoting with $\bar{X} \equiv \nabla^{c} \phi \nabla_{c} \phi$ the kinetic term, the action of general DHOST gravity is

$$
\begin{aligned}
S_{(\mathrm{DHOST})}\left[g_{a b}, \phi\right]= & \int d^{4} x \sqrt{-g}\left\{f_{0}(\phi, \bar{X})+f_{1}(\phi, \bar{X}) \square \phi+f_{2}(\phi, \bar{X}) \mathcal{R}\right. \\
& +\mathcal{A}_{(2)}^{a b c d} \nabla_{a} \nabla_{b} \phi \nabla_{c} \nabla_{d} \phi+f_{3}(\phi, \bar{X}) G_{a b} \nabla^{a} \nabla^{b} \phi \\
& \left.+\mathcal{A}_{(3)}^{a b c d e f} \nabla_{a} \nabla_{b} \phi \nabla_{c} \nabla_{d} \phi \nabla_{e} \nabla_{f} \phi\right\}
\end{aligned}
$$

where the quadratic terms are written as

$$
\mathcal{A}_{(2)}^{a b c d} \nabla_{a} \nabla_{b} \phi \nabla_{c} \nabla_{d} \phi=\sum_{i=1}^{5} \alpha_{i}(\phi, \bar{X}) \mathcal{L}_{(2)}^{i},
$$

and

$$
\begin{aligned}
& \mathcal{L}_{(2)}^{1}=\nabla_{a} \nabla_{b} \phi \nabla^{a} \nabla^{b} \phi, \\
& \mathcal{L}_{(2)}^{2}=(\square \phi)^{2}, \\
& \mathcal{L}_{(2)}^{3}=(\square \phi) \nabla^{a} \phi \nabla^{b} \phi \nabla_{a} \nabla_{b} \phi, \\
& \mathcal{L}_{(2)}^{4}=\nabla^{a} \phi \nabla_{b} \phi \nabla_{a} \nabla_{c} \phi \nabla^{c} \nabla^{b} \phi, \\
& \mathcal{L}_{(2)}^{5}=\left(\nabla^{a} \phi \nabla^{b} \phi \nabla_{a} \nabla_{b} \phi\right)^{2} .
\end{aligned}
$$

The cubic terms are written as

$$
\mathcal{A}_{(3)}^{a b c d e f} \nabla_{a} \nabla_{b} \phi \nabla_{c} \nabla_{d} \phi \nabla_{e} \nabla_{f} \phi=\sum_{i=1}^{10} \beta_{i}(\phi, \bar{X}) \mathcal{L}_{(3)}^{i},
$$

\footnotetext{
${ }^{36}$ Metric perturbations around a spherically symmetric background are decomposed into polar (i.e., even parity) and axial (i.e., odd parity) modes, as in GR. The scalar field $\phi$ appears only in the even parity perturbations.
} 
where

$$
\begin{aligned}
& \mathcal{L}_{(3)}^{1}=(\square \phi)^{3}, \\
& \mathcal{L}_{(3)}^{2}=(\square \phi) \nabla_{a} \nabla_{b} \phi \nabla^{a} \nabla^{b} \phi, \\
& \mathcal{L}_{(3)}^{3}=\nabla_{a} \nabla_{b} \phi \nabla^{b} \nabla^{c} \phi \nabla^{a} \nabla_{c} \phi, \\
& \mathcal{L}_{(3)}^{4}=(\square \phi)^{2} \nabla_{a} \phi \nabla_{b} \phi \nabla^{a} \nabla^{b} \phi, \\
& \mathcal{L}_{(3)}^{5}=(\square \phi) \nabla_{a} \phi \nabla^{c} \phi \nabla^{a} \nabla^{b} \phi \nabla_{b} \nabla_{c} \phi, \\
& \mathcal{L}_{(3)}^{6}=\left(\nabla_{a} \nabla_{b} \phi \nabla^{a} \nabla^{b} \phi\right) \nabla_{c} \phi \nabla_{d} \phi \nabla^{c} \nabla^{d} \phi, \\
& \mathcal{L}_{(3)}^{7}=\nabla_{a} \phi \nabla_{d} \phi \nabla^{a} \nabla^{b} \phi \nabla_{b} \nabla_{c} \phi \nabla^{c} \nabla^{d} \phi, \\
& \mathcal{L}_{(3)}^{8}=\nabla_{a} \phi \nabla_{c} \phi \nabla_{d} \phi \nabla_{e} \phi \nabla^{a} \nabla^{b} \phi \nabla_{b} \nabla^{c} \phi \nabla^{d} \nabla^{e} \phi, \\
& \mathcal{L}_{(3)}^{9}=(\square \phi)\left(\nabla_{a} \phi \nabla_{b} \phi \nabla^{a} \nabla^{b} \phi\right)^{2}, \\
& \mathcal{L}_{(3)}^{10}=\left(\nabla_{a} \phi \nabla_{b} \phi \nabla^{a} \nabla^{b} \phi\right)^{3} .
\end{aligned}
$$

The $\alpha_{i}(\phi, \bar{X})$ and $\beta_{i}(\phi, \bar{X})$, and therefore the $\mathcal{A}_{(2)}^{a b c d}$ and $\mathcal{A}_{(3)}^{a b c d e f}$ are arbitrary (regular) functions of their arguments. The requirement that the Ostrogradsky ghost is avoided imposes relations between these functions [561].

The EFT approach to scalar-tensor theories briefly mentioned in Sec. 13 can also be applied to the more general scenario discussed here in order to investigate the quantum stability of the proposed class of theories. Again, we refer the reader interested in the details on this topic mostly to [535] and references therein.

Naturally, spherically symmetric solutions of DHOST theories describing compact objects (and, more recently, also cylindrical solutions describing rotating objects $[562,563,564,565,566])$ have been studied. Compact star configurations were discussed in Refs. [567, 568, 569, 570, 571, 572, 573]. Subject to certain hypotheses, no-hair theorems for black holes were stated 
in [574], but soon conditions were found that allow one to circumvent them and to construct hairy black holes. The most notable way to do this consists of a scalar (hair) field that violates the staticity of the geometry through a linear dependence on time, $\phi(t, r)=q t+\psi(r)$, with $q$ a constant [384, 575, 576, 577] (a trick known from Horndeski [578, 546, 539] and beyond Horndeski $[579,580]$ gravity). Most of these DHOST geometries are solutions of the Einstein equations with vanishing stress-energy tensor of the scalar field, i.e., stealth solutions. The kinetic term $\bar{X}$ is usually constant $[581,582,583,584]$. Some of these analytic solutions arise in the earlier context of ghost condensation and are known to be immune from the strong coupling problem $[585,586,587,588]$ that usually affects constant $\bar{X}$ solutions. Stealth solutions include the Schwarzschild and the Schwarzschild-(Anti-)de Sitter spaces $[582,539,580,581,559,583,557]$ (neutron star models were also developed [569]). These stealth black hole solutions with constant kinetic term $\bar{X}$ are plagued by the problem of the strong coupling of scalar perturbations [560].

A step beyond stealth solutions was taken in Ref. [589] by using disformal transformations as a solution-generating technique to map seed solutions into new DHOST solutions. The new solutions thus obtained have nonconstant kinetic term $\bar{X}$. The metric is transformed as

$$
g_{a b} \rightarrow \tilde{g}_{a b}=A(\phi, \bar{X}) g_{a b}+B(\phi, \bar{X}) \nabla_{a} \phi \nabla_{b} \phi .
$$

For example, taking the FJNWBW geometry (51) and (52) as a seed, one obtains the static and spherically symmetric DHOST solution [589]

$$
\begin{aligned}
d \tilde{s}^{2}= & \frac{1}{A(r)}\left\{-\left(1-\frac{r_{S}}{r}\right)^{\gamma} d t^{2}+\left(1-\frac{r_{S}}{r}\right)^{-\gamma}\right. \\
& \times\left[1-\frac{q^{2}}{4 \pi r^{4}}\left(1-\frac{r_{S}}{r}\right)^{\gamma-2} B(\phi(r), \bar{X}(r))\right] d r^{2} \\
& \left.+r^{2}\left(1-\frac{r_{S}}{r}\right)^{1-\gamma} d \Omega_{(2)}^{2}\right\} \\
\bar{X}(r)= & \frac{q^{2}}{4 \pi r^{4}} \frac{A(r)\left(1-\frac{r_{S}}{r}\right)^{\gamma-2}}{1-\frac{q^{2}}{4 \pi r^{4}}\left(1-\frac{r_{S}}{r}\right)^{\gamma-2} B(\phi(r), \bar{X}(r))},
\end{aligned}
$$

where $q$ and $r_{S}>0$ are constants. This solution does not admit horizons and, like FJNWBW, it exhibits a naked singularity. In general, by disformally transforming a naked singularity one cannot obtain a black hole, but produces another naked singularity [589]. 
Other analytic black hole solutions with nonconstant $\bar{X}$ in quadratic DHOST theories, which are static and spherically symmetric but not Schwarzschildde Sitter, were found in Ref. [590] by direct integration of the equations of motion, without using the disformal transformation generating technique (see also $[559,583])$. For example, in the quadratic DHOST gravity with

$$
\begin{aligned}
f_{0}(\bar{X}) & =\eta_{1} \bar{X}^{n}, \quad f_{2}(\bar{X})=\zeta \\
\alpha_{1}(\phi, \bar{X}) & =\frac{\beta_{1} \zeta}{\bar{X}}, \quad \alpha_{2}(\phi, \bar{X})=-\alpha_{1}(\phi, \bar{X}), \quad \alpha_{3}(\phi, \bar{X})=\frac{\gamma_{1} \zeta}{\bar{X}^{2}}, \\
\alpha_{4}(\phi, \bar{X}) & =-\frac{\left[-12 \beta_{1}^{2}+16 \beta_{1}^{3}-12 \beta_{1} \gamma_{1}+\gamma_{1}\left(8+\gamma_{1}\right)\right] \zeta}{8\left(1-\beta_{1}\right)^{2} \bar{X}^{2}} \\
\alpha_{5}(\phi, \bar{X}) & =\frac{\left(2 \beta_{1}-\gamma_{1}\right)\left(2 \beta_{1}^{2}-4 \gamma_{1}+3 \beta_{1} \gamma_{1}\right) \zeta}{8\left(1-\beta_{1}\right)^{2} \bar{X}^{3}}
\end{aligned}
$$

with $\eta_{1}, \beta_{1}, \gamma_{1}$ constants, $n>0, \eta_{1}>0, \beta_{1}<0,4 n\left(1-\beta_{1}\right)>3\left(\gamma_{1}-2 \beta_{1}\right)>0$, and all the other coefficients vanishing, the black hole is [590]

$$
\begin{aligned}
d s^{2}= & -r^{2} \sqrt{\frac{\beta_{1}}{\beta_{1}-1}}\left(1-\frac{2 m}{r^{1+\sqrt{\frac{\beta_{1}}{\beta_{1}-1}}}}\right) d t^{2}+\left(1-\frac{2 m}{r^{1+\sqrt{\frac{\beta_{1}}{\beta_{1}-1}}}}\right)^{-1} d r^{2} \\
& +r^{2} d \Omega_{(2)}^{2} \\
\bar{X}= & {\left[4 \zeta \frac{-\beta_{1}+\sqrt{\beta_{1}\left(\beta_{1}-1\right)}}{3 \eta_{1} r^{2}}\right]^{1 / n} . }
\end{aligned}
$$

In the other DHOST theory with

$$
\begin{aligned}
f_{0}(\bar{X}) & =-\Lambda, \quad f_{2}(\bar{X})=\zeta-\beta_{2} \bar{X}^{n} \\
\alpha_{1}(\phi, \bar{X}) & =-\alpha_{2}(\phi, \bar{X})=0, \quad \alpha_{3}(\phi, \bar{X})=\gamma_{2} \bar{X}^{n-2} \\
\alpha_{4}(\phi, \bar{X}) & =\frac{-48 n^{2} \beta_{2}^{2} \bar{X}^{2 n}+8 n \beta_{2} \gamma_{2} \bar{X}^{2 n}+\gamma_{2} \bar{X}^{n}\left[\bar{X}^{n}\left(-8 \beta_{2}+\gamma_{2}\right)+8 \zeta\right]}{8 \bar{X}^{2}\left(\beta_{2} \bar{X}^{n}-\zeta\right)}, \\
\alpha_{5}(\phi, \bar{X}) & =\frac{\gamma_{2} \bar{X}^{2 n-3}\left(4 n \beta_{2}-\gamma_{2}\right)}{2\left(\beta_{2} \bar{X}^{n}-\zeta\right)},
\end{aligned}
$$


where $\Lambda, \beta_{2}>0, \gamma_{2}=4 n \beta_{2} / \sqrt{3}>0, \zeta$, and $n$ are constants satisfying $-\left(3 \gamma_{2}-8 n \beta_{2}\right) \Lambda>0$, the DHOST black hole is [590]

$$
\begin{aligned}
d s^{2} & =-r^{2(\sqrt{3}-1)}\left(1-\frac{2 m}{r^{2+\sqrt{3}}}\right) d t^{2}+\left(1-\frac{2 m}{r^{2+\sqrt{3}}}\right)^{-1} d r^{2}+r^{2} d \Omega_{(2)}^{2} \\
\bar{X} & =\left(\frac{4 \zeta+(2 \sqrt{3}-3) \Lambda r^{2}}{4 \beta_{2}}\right)^{1 / n}
\end{aligned}
$$

A third solution for a different quadratic DHOST model is provided in [590].

In all these models, the equation locating the event horizons $\nabla^{c} r \nabla_{c} r=$ $g^{r r}=0$ (where $r$ is the areal radius) has a single root, identifying clearly a black hole horizon (as is clear from the dimensions, the constant $m$ is not the black hole mass, but a power of it). However, these black holes are not asymptotically flat [590], which can be seen as a consequence of the fact that the black hole exterior is not vacuum but hosts the scalar hair. Stability against odd parity perturbations is studied in [590], with the result that the black hole (316) is stable, while the geometry (322) is not.

The search for static and spherical DHOST solutions continues (e.g., $[591,592,593,594,595,596])$. Non-constant $\bar{X}$ solutions are useful when attempting to confront DHOST theories with observations, which has already led to significant restrictions on these theories. The reader should be warned that this area of research is very recent and developing fast.

\section{Summary and conclusions}

It is apparent from our analysis that, even in the simplest theory of relativistic gravity, GR, and under an assumption as simplifying as spherical symmetry (which is indeed, an oversimplification in most situations of astrophysical interest), only a restricted number of analytic solutions have been discovered, which leads to the usefulness of compiling a small catalogue. This richness is due to the variety of matter sources and of possible boundary conditions (asymptotically flat, FLRW, de Sitter, etc.). What is more, our current knowledge of exact solutions is incomplete and there are very few cases in which a geometry can be called "generic" in some sense, like the Schwarzschild solution in vacuum and asymptotically flat GR. Here we have refrained from entering into great detail about the concept of "generic", which will leave the more mathematically oriented reader dissatisfied, and we have focussed on the physical aspects of these solutions. Likewise, we 
have privileged the description of the geometries examined in the most wellknown and used coordinate systems and we have not entered the discussion of special algebraic properties and classifications of the solutions examined, as done instead in the well known book by Stephani et al. [44] discussing solutions of the Einstein equations of many different natures. This kind of book-length analysis is beyond the scopes of the present work and, although necessary for an invariant characterization of the geometries, ultimately does not serve well the purposes of the physicist or the cosmologist. Some of the spacetimes described here have some kind of "generic" character as described in the text, whereas those that do not have been reported as interesting examples that still show certain properties of the non-linear theories that they solve. A curious feature of certain geometries is that they solve the field equations of different theories, or of the same theory with different matter sources. This largely unexplored (beyond the KSdS and the McVittie spaces) aspect deserves more attention in future analyses.

Most likely, some of the analytic solutions presented here do not describe objects found in nature. Nevertheless, they provide useful insight into the nature of the field equations that they satisfy, for which it is difficult to build intuition due to their non-linearity. Other times, these geometries are useful as toy models for theoreticians (for example, the McVittie and non-rotating Thakurta solutions as toy models for primordial black holes [41, 42], the subject of renewed attention in relation with the dark matter problem and black hole mergers detected by $L I G O)$.

Given the enormous motivation for the study of alternative theories of gravity coming from cosmology [23], quantum corrections to gravity [3, 4, $5]$, the low-energy limit of string theories [370,371], and the experimental efforts to test gravity at various astrophysical scales, we have included scalartensor [29, 53, 54, 55], as well as Horndeski gravity and DHOST theories beyond Horndeski in our discussion. The reasons for choosing those theories include the fact that scalar-tensor gravity is the prototypical and longest known alternative to GR. Furthermore, it includes $f(\mathcal{R})$ gravity as a subclass which is extremely popular to explain the current acceleration of the universe without invoking dark energy $[24,25,26,27,28]$ and is also intimately connected with early universe inflation [6]. Whether $f(\mathcal{R})$ gravity is a toy model or, as many authors believe, a realistic model, or an effective field theory [597, 598, 599], is an open question.

Horndeski theory is arguably the most well studied recent extension of "simple" scalar-tensor gravity which is still well motivated from the physical point of view and useful for cosmology, while much work is presently being done on the more recent DHOST theories, for which it is fair to say that 
we do not yet have a complete picture. The recent opening of gravitational wave astronomy $[30,31,32]$ and the imaging of the near-horizon region of black holes [33, 34, 35, 36, 37, 38], together with the birth of multi-messenger astrophysics [600] offer new tools to explore the nature of gravity, and a clear picture of what one should expect beyond Einstein theory could help or even guide these observational efforts.

The presence of a scalar degree of freedom, either as a matter field or as part of the gravitational field, has been a recurring element in this work. Unfortunately, even for this simplest degree of freedom of field theory, our knowledge is still limited. Matter scalar fields in GR can collapse to black holes, which are then indistinguishable from any other black hole with the same mass and angular momentum, according to the no-hair theorems. The exact spherical, static, and asymptotically flat solution of the Einstein equations which does not contain a black hole, the FJNWBW solution, exhibits a naked singularity. Generally speaking, asymptotically flat analytic solutions of GR with non-trivial scalar field configurations tend to generate geometries that contain only naked singularities or wormhole (horizon) throats, but not black hole horizons. This conclusion seems to apply to both static and time-dependent geometries, when gravitational collapse does not occur. Non-asymptotically flat solutions can have a rich structure of apparent horizons which can change in number and usually appear/disappear in pairs. The same conclusions apply, in general, to scalar-tensor gravity, although there is no specific theorem to this regard. A sore point is the lack of general theorems for GR or scalar-tensor (including Horndeski and DHOST) solutions which are spherical and asymptotically FLRW.

Changing source type, many exact solutions sourced by perfect or imperfect fluids are known, especially in the form of perfect fluid stellar configurations, but only relatively few are physical. Then, there are families of solutions of GR and scalar-tensor gravity interpreted as central objects embedded in FLRW universes, some of which are black holes (as defined by their apparent horizons) at least part of the time. Other solutions contain naked singularities for at least part of the history of the universe. Apparent horizons that are also the throats of (non-traversable) wormholes are ubiquitous.

We have pointed out existing relations between many pairs of geometries listed here. Usually, these relations are conformal dualities, due to the frequent use of conformal transformations as solution-generating techniques. Other times, researchers have sought to generalize previously known solutions, which results in some of the spacetimes examined being special cases of previous ones. 
In spite of the fact that, in the space available, it is impossible to make justice to all the physical and mathematical aspects involved, or even to compile a complete list of all related references, this review is meant to provide a reference for the researcher and an introduction to the subject for the less experienced reader. A review can never replace a thorough study of the references relevant to a particular aspect or a specific geometry. The usefulness of simpler situations is that they can be kept in mind and used as paradigms, or for contrast, when studying more complicated ones. They are particularly useful in the attempt to discover what a new theory of gravity adds to the existing ones, and as examples or counterexamples. We have learned much physics in the past decades of studying analytic solutions of GR and scalar-tensor gravity but, remarkably, there are many areas in which we still know very little. We hope that highlighting them will renew research efforts that will bring a better understanding of important aspects of gravity.

\section{Acknowledgments}

We are grateful to two referees for numerous helpful suggestions and comments. We are indebted with many colleagues - too many to list - for discussions, comments, and references and to Vincenzo Vitagliano for drawing Fig. 1. This work is supported by the Natural Sciences \& Engineering Research Council of Canada (Grant No. 2016-03803 to V.F.) and by Bishop's University. This research was supported, in part, by Perimeter Institute for Theoretical Physics. Research at Perimeter Institute is supported by the Government of Canada through the Department of Innovation, Science and Economic Development Canada and by the Province of Ontario through the Ministry of Research, Innovation and Science.

A.G. is supported by the European Union's Horizon 2020 research and innovation programme under the Marie Skłodowska-Curie Actions (grant agreement No. 895648 - CosmoDEC). His work has been carried out in the framework of the activities of the Italian National Group for Mathematical Physics [Gruppo Nazionale per la Fisica Matematica (GNFM), Istituto Nazionale di Alta Matematica (INdAM)].

\section{References}

[1] H. Weyl, Eine neue erweiterung der relativitaetstheorie, Ann. Phys. (Leipzig) 364 (1919) 101-133. 
[2] A. S. Eddington, The Mathematical Theory of Relativity, Cambridge University Press, Cambridge, UK, 1923.

[3] R. Utiyama, B. S. DeWitt, Renormalization of a classical gravitational field interacting with quantized matter fields, J. Math. Phys. 3 (1962) 608-618.

[4] K. S. Stelle, Renormalization of higher-derivative quantum gravity, Phys. Rev. D 16 (1977) 953.

[5] K. S. Stelle, Classical gravity with higher derivatives, Gen. Relativ. Gravit. 9 (1978) 353-371.

[6] A. A. Starobinsky, A new type of isotropic cosmological models without singularity, Phys. Lett. B 91 (1980) 99-102.

[7] A. G. Riess, et al., Observational evidence from supernovae for an accelerating universe and a cosmological constant, Astron. J. 116 (1998) 1009-1038. arXiv:astro-ph/9805201, doi:10.1086/300499.

[8] A. G. Riess, A. V. Filippenko, W. Li, R. R. Treffers, B. P. Schmidt, Y. Qiu, J. Hu, M. Armstrong, C. Faranda, E. Thouvenot, The risetime of nearby type Ia supernovae, Astron. J. 118 (1999) 2675-2688. arXiv:astro-ph/9907037, doi:10.1086/301143.

[9] A. G. Riess, et al., Type Ia supernova discoveries at $z>1$ from the Hubble Space Telescope: Evidence for past deceleration and constraints on dark energy evolution, Astrophys. J. 607 (2004) 665-687. arXiv:astro-ph/0402512, doi:10.1086/383612.

[10] D. J. Eisenstein, et al., Detection of the Baryon Acoustic Peak in the Large-Scale Correlation Function of SDSS Luminous Red Galaxies, Astrophys. J. 633 (2005) 560-574. arXiv:astro-ph/0501171, doi:10.1086/466512.

[11] P. Astier, et al., The supernova legacy survey: measurement of $\Omega_{M}, \Omega_{\Lambda}$ and $w$ from the first year data set, Astron. Astrophys. 447 (2006) 3148 .

[12] D. N. Spergel, et al., Wilkinson Microwave Anisotropy Probe (WMAP) three year results: implications for cosmology, Astrophys. J. Suppl. 170 (2007) 377. arXiv:astro-ph/0603449, doi:10.1086/513700. 
[13] S. Weinberg, The Cosmological Constant Problem, Rev. Mod. Phys. 61 (1989) 1-23. doi:10.1103/RevModPhys.61.1.

[14] S. M. Carroll, The cosmological constant, Living Rev. Relativ. 4 (2001) 1. arXiv:astro-ph/0004075, doi:10.12942/lrr-2001-1.

[15] P. J. E. Peebles, B. Ratra, Cosmology with a time-variable cosmological'constant', Astrophys. J. 325 (1988) L17-L20.

[16] B. Ratra, P. J. Peebles, Cosmological consequences of a rolling homogeneous scalar field, Phys. Rev. D 37 (1988) 3406.

[17] C. Wetterich, Cosmology and the Fate of Dilatation Symmetry, Nucl. Phys. B 302 (1988) 668-696. arXiv:1711.03844, doi:10.1016/05503213(88)90193-9.

[18] J. P. Ostriker, P. J. Steinhardt, The observational case for a lowdensity universe with a non-zero cosmological constant, Nature 377 (1995) 600-602.

[19] R. Caldwell, R. Dave, P. J. Steinhardt, Cosmological imprint of an energy component with general equation of state, Phys. Rev. Lett. 80 (1998) 1582-1585. arXiv:astro-ph/9708069, doi:10.1103/PhysRevLett.80.1582.

[20] S. M. Carroll, Quintessence and the rest of the world, Phys. Rev. Lett. 81 (1998) 3067-3070. arXiv:astro-ph/9806099, doi:10.1103/PhysRevLett.81.3067.

[21] N. A. Bahcall, J. P. Ostriker, S. Perlmutter, P. J. Steinhardt, The Cosmic triangle: Assessing the state of the universe, Science 284 (1999) 1481-1488. arXiv:astro-ph/9906463, doi:10.1126/science.284.5419.1481.

[22] L. Wang, R. R. Caldwell, J. P. Ostriker, P. J. Steinhardt, Cosmic concordance and quintessence, Astrophys. J. 530 (2000) 17.

[23] L. Amendola, S. Tsujikawa, Dark Energy: Theory and Observations, Cambridge University Press, Cambridge, UK, 2010.

[24] S. Capozziello, S. Carloni, A. Troisi, Quintessence without scalar fields, Recent Res. Dev. Astron. Astrophys. 1 (2003) 625. arXiv:astroph/0303041. 
[25] S. M. Carroll, V. Duvvuri, M. Trodden, M. S. Turner, Is cosmic speedup due to new gravitational physics?, Phys. Rev. D 70 (2004) 043528. arXiv:astro-ph/0306438, doi:10.1103/PhysRevD.70.043528.

[26] T. P. Sotiriou, V. Faraoni, $f(R)$ theories of gravity, Rev. Mod. Phys. $82(2010) 451$.

[27] A. De Felice, S. Tsujikawa, Cosmology of a covariant galileon field, Phys. Rev. Lett. 105 (2010) 111301.

[28] S. Nojiri, S. D. Odintsov, Unified cosmic history in modified gravity: from $f(R)$ theory to Lorentz non-invariant models, Phys. Repts. 505 (2011) 59-144.

[29] C. Brans, R. H. Dicke, Mach's principle and a relativistic theory of gravitation, Phys. Rev. 124 (1961) 925.

[30] B. P. Abbott, et al., Observation of Gravitational Waves from a Binary Black Hole Merger, Phys. Rev. Lett. 116 (2016) 061102. arXiv:1602.03837, doi:10.1103/PhysRevLett.116.061102.

[31] B. P. Abbott, et al., GW151226: Observation of Gravitational Waves from a 22-Solar-Mass Binary Black Hole Coalescence, Phys. Rev. Lett. 116 (2016) 241103. arXiv:1606.04855, doi:10.1103/PhysRevLett.116.241103.

[32] B. P. Abbott, et al., GW170104: Observation of a 50-Solar-Mass Binary Black Hole Coalescence at Redshift 0.2, Phys. Rev. Lett. 118 (2017) 221101, [Erratum: Phys. Rev. Lett. 121, 129901 (2018)]. arXiv:1706.01812, doi:10.1103/PhysRevLett.118.221101.

[33] K. Akiyama, et al., First M87 Event Horizon Telescope results. I. The shadow of the supermassive black hole, Astrophys. J. (Lett.) 875 (2019) L1.

[34] R. Azulay, et al., First M87 Event Horizon Telescope results. II. Array and instrumentation, Astrophys. J. (Lett.) 875 (2019) 28pp.

[35] K. Akiyama, et al., First M87 Event Horizon Telescope results. III. Data processing and calibration, Astrophys. J. (Lett.) 875 (2019) L3.

[36] K. Akiyama, et al., First M87 Event Horizon Telescope results. IV. Imaging the central supermassive black hole, Astrophys. J. (Lett.) 875 (2019) L4. 
[37] K. Akiyama, et al., First M87 Event Horizon Telescope results. V. Physical origin of the asymmetric ring, Astrophys. J. (Lett.) 875 (2019) L5.

[38] K. Akiyama, et al., First M87 Event Horizon Telescope results. VI. The shadow and mass of the central black hole, Astrophys. J. (Lett.) 875 (2019) L6.

[39] M. Sasaki, T. Suyama, T. Tanaka, S. Yokoyama, Primordial black hole scenario for the gravitational-wave event GW150914, Phys. Rev. Lett. 117 (2016) 061101.

[40] B. Carr, J. Silk, Primordial black holes as generators of cosmic structures, Mon. Not. Roy. Astron. Soc. 478 (2018) 3756-3775.

[41] C. Boehm, A. Kobakhidze, C. A. J. O’hare, Z. S. C. Picker, M. Sakellariadou, Eliminating the LIGO bounds on primordial black hole dark matter, JCAP 03 (2021) 078. arXiv:2008.10743, doi:10.1088/1475$7516 / 2021 / 03 / 078$.

[42] F. Ruiz, C. Molina, J. A. S. Lima, Dynamical model for primordial black holes, Phys. Rev. D 102 (2020) 123516.

[43] K. Schwarzschild, Über das gravitationsfeld einer kugel aus inkompressibler flüssigkeit nach der Einsteinschen theorie, Sitz. Deut. Akad. Wiss. Math.Phys. Berlin 24 (1916) 424-434.

[44] H. Stephani, D. Kramer, M. MacCallum, C. Hoenselaers, E. Herlt, Exact Solutions of Einstein's Field Equations, Cambridge University Press, Cambridge, UK, 2009.

[45] A. Krasiński, Inhomogeneous Cosmological Models, Cambridge University Press, Cambridge, UK, 2006.

[46] J. B. Griffiths, J. Podolskỳ, Exact Space-Times in Einstein's General Relativity, Cambridge University Press, Cambridge, UK, 2009.

[47] V. Faraoni, Cosmological and Black Hole Apparent Horizons, Springer, New York, 2015.

[48] V. Faraoni, Embedding black holes and other inhomogeneities in the universe in various theories of gravity: A short review, Universe 4 (2018) 109. 
[49] D. Tretyakova, B. Latosh, Scalar-tensor black holes embedded in an expanding universe, Universe 4 (2018) 26.

[50] R. M. Wald, General Relativity, Chicago University Press, Chicago, 1984.

[51] G. Aad, et al., Observation of a new particle in the search for the Standard Model Higgs boson with the ATLAS detector at the LHC, Phys. Lett. B 716 (2012) 1-29. arXiv:1207.7214, doi:10.1016/j.physletb.2012.08.020.

[52] S. Chatrchyan, et al., Observation of a New Boson at a Mass of 125 GeV with the CMS Experiment at the LHC, Phys. Lett. B 716 (2012) 30-61. arXiv:1207.7235, doi:10.1016/j.physletb.2012.08.021.

[53] P. G. Bergmann, Comments on the scalar tensor theory, Int. J. Theor. Phys. 1 (1968) 25-36. doi:10.1007/BF00668828.

[54] R. V. Wagoner, Scalar-tensor theory and gravitational waves, Phys. Rev. D 1 (1970) 3209.

[55] K. J. Nordtvedt, Post-Newtonian metric for a general class of scalartensor gravitational theories and observational consequences, Astrophys. J. 161 (1970) 1059.

[56] G. W. Horndeski, Second-order scalar-tensor field equations in a four-dimensional space, Int. J. Theor. Phys. 10 (1974) 363-384. doi:10.1007/BF01807638.

[57] J. Gleyzes, D. Langlois, F. Piazza, F. Vernizzi, Healthy theories beyond Horndeski, Phys. Rev. Lett. 114 (21) (2015) 211101. arXiv:1404.6495, doi:10.1103/PhysRevLett.114.211101.

[58] J. Gleyzes, D. Langlois, F. Piazza, F. Vernizzi, Exploring gravitational theories beyond Horndeski, JCAP 02 (2015) 018. arXiv:1408.1952, doi:10.1088/1475-7516/2015/02/018.

[59] R. Emparan, H. S. Reall, Black holes in higher dimensions, Living Rev. Relativ. 11 (2008) 6.

[60] G. T. Horowitz, Black Holes In Higher Dimensions, Cambridge University Press, Cambridge, UK, 2012.

[61] I. Booth, Black hole boundaries, Can. J. Phys. 83 (2005) 1073-1099. arXiv:gr-qc/0508107, doi:10.1139/p05-063. 
[62] A. B. Nielsen, Black holes and black hole thermodynamics without event horizons, Gen. Relativ. Gravit. 41 (2009) 1539-1584.

[63] R. M. Wald, V. Iyer, Trapped surfaces in the Schwarzschild geometry and cosmic censorship, Phys. Rev. D 44 (1991) 3719-3722. doi:10.1103/PhysRevD.44.R3719.

[64] E. Schnetter, B. Krishnan, Non-symmetric trapped surfaces in the Schwarzschild and Vaidya spacetimes, Phys. Rev. D 73 (2006) 021502. arXiv:gr-qc/0511017, doi:10.1103/PhysRevD.73.021502.

[65] V. Faraoni, G. F. R. Ellis, J. T. Firouzjaee, A. Helou, I. Musco, Foliation dependence of black hole apparent horizons in spherical symmetry, Phys. Rev. D 95 (2017) 024008. arXiv:1610.05822, doi:10.1103/PhysRevD.95.024008.

[66] S. Weinberg, Gravitation and Cosmology: Principles and Applications of The General Theory of Relativity, Wiley, New York, 1972.

[67] H. Bondi, C. W. Kilmister, Note on Schiff's paper on relativity, Am. J. Phys. 28 (1960) 508-508.

[68] J. D. French, Pedagogical trick for general relativity, Am. J. Phys. 45 (1977) 580.

[69] T. Jacobson, When is $g(t t) g(r r)=-1$ ?, Class. Quantum Grav. 24 (2007) 5717-5719. arXiv:0707.3222, doi:10.1088/0264$9381 / 24 / 22 / \mathrm{N} 02$.

[70] M. Barriola, A. Vilenkin, Gravitational field of a global monopole, Phys. Rev. Lett. 63 (1989) 341.

[71] A. B. Nielsen, M. Visser, Production and decay of evolving horizons, Class. Quantum Grav. 23 (2006) 4637.

[72] G. Abreu, M. Visser, Kodama time: Geometrically preferred foliations of spherically symmetric spacetimes, Phys. Rev. D 82 (2010) 044027. arXiv:1004.1456, doi:10.1103/PhysRevD.82.044027.

[73] C. W. Misner, D. H. Sharp, Relativistic equations for adiabatic, spherically symmetric gravitational collapse, Phys. Rev. 136 (1964) B571.

[74] W. C. Hernandez, C. W. Misner, Observer time as a coordinate in relativistic spherical hydrodynamics, Astrophys. J. 143 (1966) 452. 
[75] B. Paczynsky, P. J. Wiita, Thick accretion disks and supercritical luminosities, Astron. Astrophys. 88 (1980) 23-31.

[76] J. Kovár, Z. Stuchlík, P. Slaný, in: Z. Stuchlík, G. Török, T. Pecháček (Eds.), RAGtime 4/5: Proceedings of Workshops on Black Holes and Neutron Stars: 14-16/13-15 October 2002/2003 Opava, Czech Republic, Silesian University, Opava, Czech Republic, 2014, p. 133.

[77] M. A. Abramowicz, The Paczynski-Wiita potential: A step-by-step 'derivation', Astron. Astrophys. 500 (2009) 213. arXiv:0904.0913, doi:10.1051/0004-6361/200912155.

[78] M. A. Abramowicz, M. Calvani, L. Nobili, Thick accretion disks with super-Eddington luminosities, Astrophys. J. 242 (1980) 772.

[79] M. A. Nowak, R. V. Wagoner, Diskoseismology: Probing accretion disks. I. Trapped adiabatic oscillations, Astrophys. J. 378 (1991) 656664 .

[80] I. V. Artemova, G. Björnsson, I. D. Novikov, Modified Newtonian potentials for the description of relativistic effects in accretion disks around black holes, Astrophys. J. 461 (1996) 565.

[81] O. Semerák, V. Karas, Pseudo-Newtonian models of a rotating black hole field (1999). arXiv:astro-ph/9901289.

[82] W. Kluźniak, W. H. Lee, The swallowing of a quark star by a black hole, Mon. Not. Roy. Astron. Soc. 335 (2002) L29-L32.

[83] B. Mukhopadhyay, R. Misra, Pseudo-Newtonian potentials to describe the temporal effects on relativistic accretion disks around rotating black holes and neutron stars, Astrophys. J. 582 (2003) 347.

[84] R. I. Ivanov, E. M. Prodanov, Pseudo-Newtonian potential for charged particle in Kerr-Newman geometry, Phys. Lett. B 611 (2005) 34-38. arXiv:gr-qc/0504025, doi:10.1016/j.physletb.2005.02.047.

[85] S. K. Chakrabarti, S. Mondal, Studies of accretion flows around rotating black holes I. Particle dynamics in a pseudo-Kerr potential, Mon. Not. Roy. Astron. Soc. 369 (2006) 976-984.

[86] S. Ghosh, B. Mukhopadhyay, Generalized pseudo-Newtonian potential for studying accretion disk dynamics in off-equatorial planes around rotating black holes: Description of a vector potential, Astrophys. J. 667 (2007) 367-374. arXiv:0706.2221, doi:10.1086/520920. 
[87] C. Wegg, Pseudo-Newtonian potentials for nearly parabolic orbits, Astrophys. J. 749 (2012) 183.

[88] E. Tejeda, S. Rosswog, Generalized Newtonian description of particle motion in spherically symmetric spacetimes (2014). arXiv:1402.1171.

[89] V. Witzany, O. Semerák, P. Suková, Free motion around black holes with discs or rings: between integrability and chaos IV, Mon. Not. Roy. Astron. Soc. 451 (2015) 1770-1794.

[90] V. Faraoni, S. D. Belknap-Keet, M. Lapierre-Léonard, PaczynskiWiita-like potential for any static spherical black hole in metric theories of gravity, Phys. Rev. D 93 (2016) 044042.

[91] J. T. Jebsen, On the general spherically symmetric solutions of Einstein's gravitational equations in vacuo, Gen. Relativ. Gravit. 37 (2005) 2253-2259.

[92] G. D. Birkhoff, R. E. Langer, Relativity and Modern Physics, Harvard University Press, Harvard, USA, 1923.

[93] K. A. Bronnikov, V. N. Melnikov, The Birkhoff theorem in multidimensional gravity, Gen. Rel. Grav. 27 (1995) 465-474. arXiv:grqc/9403063, doi:10.1007/BF02105073.

[94] G. W. Gibbons, D. Ida, T. Shiromizu, Uniqueness and nonuniqueness of static vacuum black holes in higher dimensions, Prog. Theor. Phys. Suppl. 148 (2003) 284-290. arXiv:gr-qc/0203004, doi:10.1143/PTPS.148.284.

[95] G. W. Gibbons, D. Ida, T. Shiromizu, Uniqueness and nonuniqueness of static black holes in higher dimensions, Phys. Rev. Lett. 89 (2002) 041101. arXiv:hep-th/0206049, doi:10.1103/PhysRevLett.89.041101.

[96] P. T. Chrusciel, J. Lopes Costa, M. Heusler, Stationary Black Holes: Uniqueness and Beyond, Living Rev. Relativ. 15 (2012) 7. arXiv:1205.6112, doi:10.12942/lrr-2012-7.

[97] A. Das, Birkhoff's theorem for electro-magnetic fields in general relativity, Prog. Theor. Phys. 24 (1960) 915-916.

[98] G. V. Isaev, JINR preprint P2-10347 (Dubna) (1976). 
[99] K. A. Bronnikov, M. A. Kovalchuk, N. V. Pavlov, in: K. P. Staniukovich (Ed.), Problemy Theorii Gravitasii i Elementarykh Chastits, Atomindat, Moskow [in Russian], 1976.

[100] K. A. Bronnikov, M. A. Kovalchuk, On a generalisation of Birkhoff's theorem, J. Phys. A 13 (1980) 187.

[101] R. Goswami, G. F. R. Ellis, Almost Birkhoff theorem in general relativity, Gen. Relativ. Gravit. 43 (2011) 2157-2170.

[102] R. Goswami, G. F. R. Ellis, Birkhoff theorem and matter, Gen. Relativ. Gravit. 44 (2012) 2037-2050.

[103] D. B. Sibandze, R. Goswami, S. D. Maharaj, A. M. Nzioki, P. K. S. Dunsby, Scalar wave scattering from Schwarzschild black holes in modified gravity (2016). arXiv:1611.06043.

[104] A. M. Nzioki, R. Goswami, P. K. S. Dunsby, Jebsen-Birkhoff theorem and its stability in $f(R)$ gravity, Phys. Rev. D 89 (2014) 064050.

[105] F. Kottler, Über die physikalischen grundlagen der Einsteinschen gravitationstheorie, Ann. Phys. (Leipzig) 361 (1918) 401-462.

[106] H.-J. Schmidt, The tetralogy of Birkhoff theorems, Gen. Relativ. Gravit. 45 (2013) 395-410.

[107] A. C. Fabian, A. N. Lasenby, in: C. Rovelli (Ed.), General Relativity: The Most Beautiful of Theories, Vol. 28, De Gruyter, Berlin, 2015.

[108] J. L. Synge, Relativity: The General Theory, North-Holland, Amsterdam, 1960.

[109] K. Schleich, D. M. Witt, A simple proof of Birkhoff's theorem for cosmological constant, J. Math. Phys. 51 (2010) 112502. arXiv:0908.4110, doi:10.1063/1.3503447.

[110] W. Boucher, G. W. Gibbons, G. T. Horowitz, A Uniqueness Theorem for Anti-de Sitter Space-time, Phys. Rev. D 30 (1984) 2447. doi:10.1103/PhysRevD.30.2447.

[111] H. Kodama, Uniqueness and stability of higher-dimensional black holes (2004). arXiv:hep-th/0403030.

[112] P. G. LeFloch, L. Rozoy, Uniqueness of Kottler spacetime and Besse conjecture (2010). arXiv:1009.0936. 
[113] A. K. M. Masood-ul Alam, W. Yu, Uniqueness of de Sitter and Schwarzschild-de Sitter spacetimes, Comm. Analysis \& Geometry 23 (2015) 377-387.

[114] J. Guven, D. Núñez, Schwarzschild-de Sitter space and its perturbations, Phys. Rev. D 42 (1990) 2577-2584. doi:10.1103/physrevd.42.2577.

[115] R. Balbinot, E. Poisson, Stability of the Schwarzschild-de Sitter model, Phys. Rev. D 41 (1990) 395-402. doi:10.1103/PhysRevD.41.395.

[116] F. Mellor, I. Moss, Stability of Black Holes in de Sitter Space, Phys. Rev. D 41 (1990) 403. doi:10.1103/PhysRevD.41.403.

[117] H. Otsuki, T. Futamase, Gravitational perturbation of Schwarzschildde Sitter spacetime and its quasi-normal modes, Prog. Theor. Phys. 85 (1991) 771-778.

[118] A. H. Abbassi, Nonstatic spherically symmetric solution of Einstein vacuum field equations with Lambda, J. High Energy Phys. 04 (1999) 011. arXiv:gr-qc/9902009, doi:10.1088/1126-6708/1999/04/011.

[119] A. H. Abbassi, S. Gharanfoli, A. M. Abbassi, General spherically symmetric solutions of Einstein vacuum fields equations with Lambda, Apeiron 9 (2002) 1.

[120] K. A. Meissner, Horizons and the cosmological constant (2009). arXiv:0901.0640.

[121] P. Castelo Ferreira, A Locally Anisotropic Metric for Matter in an Expanding Universe: I. The Ansatz and the Modified Newton Law (2009). arXiv:0907.0847.

[122] P. Castelo Ferreira, An expanding locally anisotropic (ELA) metric describing matter in an expanding universe, Phys. Lett. B 684 (2010) 73-76. arXiv:1006.1617, doi:10.1016/j.physletb.2010.01.001.

[123] P. Castelo Ferreira, On the variation of the Astronomical Unit and the corrections to planetary motion on an expanding locally anisotropic background, Acta Appl. Math. 131 (2014) 155-177. arXiv:1203.1844, doi:10.1007/s10440-013-9852-1.

[124] P. Castelo Ferreira, Constraining an Expanding Locally Anisotropic metric from the Pioneer anomaly, Adv. Space Res. 51 (2013) 12661277. arXiv:1202.6189, doi:10.1016/j.asr.2012.11.004. 
[125] V. Faraoni, A. M. Cardini, W.-J. Chung, Simultaneous baldness and cosmic baldness and the Kottler spacetime, Phys. Rev. D 97 (2018) 024046 .

[126] R. M. Wald, Asymptotic behavior of homogeneous cosmological models in the presence of a positive cosmological constant, Phys. Rev. D 28 (1983) 2118.

[127] M. S. Turner, L. M. Widrow, Homogeneous cosmological models and new inflation, Phys. Rev. Lett. 57 (1986) 2237.

[128] L. G. Jensen, J. A. Stein-Schabes, Is inflation natural?, Phys. Rev. D 35 (1987) 1146.

[129] J. Chase, Event horizons in static scalar-vacuum space-times, Comm. Math. Phys. 19 (1970) 276-288.

[130] R. Ruffini, J. A. Wheeler, Introducing the black hole, Phys. Today 24 (1971) 30. doi:10.1063/1.3022513.

[131] J. D. Bekenstein, Nonexistence of baryon number for static black holes, Phys. Rev. D 5 (1972) 1239-1246. doi:10.1103/PhysRevD.5.1239.

[132] J. D. Bekenstein, Nonexistence of baryon number for black holes. II, Phys. Rev. D 5 (1972) 2403-2412. doi:10.1103/PhysRevD.5.2403.

[133] J. D. Bekenstein, Transcendence of the law of baryon-number conservation in black hole physics, Phys. Rev. Lett. 28 (1972) 452-455. doi:10.1103/PhysRevLett.28.452.

[134] J. D. Bekenstein, Black hole hair: twenty-five years after (1996). arXiv:gr-qc/9605059.

[135] C. Teitelboim, Nonmeasurability of the lepton number of a black hole, Lett. Nuovo Cimento 3 (1972) 397-400.

[136] T. Zannias, Black holes cannot support conformal scalar hair, J. Math. Phys. 36 (1995) 6970-6980.

[137] J. D. Bekenstein, Novel "no-scalar-hair" theorem for black holes, Phys. Rev. D 51 (1995) 6608. doi:10.1103/PhysRevD.51.R6608.

[138] A. Saa, New no-scalar-hair theorem for black holes, J. Math. Phys. 37 (1996) 2346-2351. 
[139] K. A. Bronnikov, Spherically symmetric false vacuum: No go theorems and global structure, Phys. Rev. D 64 (2001) 064013. arXiv:grqc/0104092, doi:10.1103/PhysRevD.64.064013.

[140] C. A. Herdeiro, E. Radu, Asymptotically flat black holes with scalar hair: a review, Int. J. Mod. Phys. D 24 (2015) 1542014. arXiv:1504.08209, doi:10.1142/S0218271815420146.

[141] T. P. Sotiriou, Black Holes and Scalar Fields, Class. Quantum Grav. 32 (2015) 214002. arXiv:1505.00248, doi:10.1088/0264$9381 / 32 / 21 / 214002$.

[142] D. Christodoulou, The instability of naked singularities in the gravitational collapse of a scalar field, Ann. Math. 149 (1999) 183-217.

[143] J. Liu, J. Li, A robust proof of the instability of naked singularities of a scalar field in spherical symmetry, Comm. Math. Phys. 363 (2018) $561-578$.

[144] D. S. Goldwirth, T. Piran, Gravitational Collapse of Massless Scalar Field and Cosmic Censorship, Phys. Rev. D 36 (1987) 3575. doi:10.1103/PhysRevD.36.3575.

[145] P. S. Joshi, Global Aspects In Gravitation and Cosmology, Clarendon Press, Oxford, UK, 1993.

[146] P. S. Joshi, The rainbows of gravity (2013). arXiv:1305.1005.

[147] M. Heusler, A no-hair theorem for self-gravitating nonlinear sigma models, J. Math. Phys. 33 (1992) 3497-3502.

[148] D. Sudarsky, A simple proof of a no-hair theorem in Einstein-Higgs theory, Class. Quantum Grav. 12 (1995) 579.

[149] A. E. Mayo, J. D. Bekenstein, No hair for spherical black holes: charged and nonminimally coupled scalar field with self-interaction, Phys. Rev. D 54 (1996) 5059.

[150] M. S. Volkov, D. V. Gal'Tsov, Non-Abelian Einstein-Yang-Mills black holes., JETP Lett. 50 (1989) 346-350.

[151] P. Bizon, Colored black holes, Phys. Rev. Lett. 64 (1990) 2844.

[152] H. Künzle, A. Masood-ul Alam, Spherically symmetric static SU(2) Einstein-Yang-Mills fields, J. Math. Phys. 31 (1990) 928-935. 
[153] P. Bizon, T. Chmaj, Gravitating skyrmions, Phys. Lett. B 297 (1992) $55-62$.

[154] S. Droz, M. Heusler, N. Straumann, New black hole solutions with hair, Phys. Lett. B 268 (1991) 371-376.

[155] G. Lavrelashvili, D. Maison, Regular and black hole solutions of Einstein-Yang-Mills dilaton theory, Nucl. Phys. B 410 (1993) 407-422.

[156] T. Torii, K. Maeda, Black holes with non-Abelian hair and their thermodynamical properties, Phys. Rev. D 48 (1993) 1643.

[157] B. R. Greene, S. D. Mathur, C. M. O'Neill, Eluding the no-hair conjecture: Black holes in spontaneously broken gauge theories, Phys. Rev. D 47 (1993) 2242.

[158] K. Lee, V. Nair, E. J. Weinberg, Black holes in magnetic monopoles, Phys. Rev. D 45 (1992) 2751.

[159] W. Israel, Event horizons in static vacuum space-times, Phys. Rev. 164 (1967) 1776-1779. doi:10.1103/PhysRev.164.1776.

[160] W. Israel, Event horizons in static electrovac space-times, Comm. Math. Phys. 8 (1968) 245-260. doi:10.1007/BF01645859.

[161] S. W. Hawking, G. F. R. Ellis, The Large Scale Structure of SpaceTime, Cambridge Monographs on Mathematical Physics, Cambridge University Press, 2011. doi:10.1017/CBO9780511524646.

[162] S. Hawking, Black holes in general relativity, Comm. Math. Phys. 25 (1972) 152-166. doi:10.1007/BF01877517.

[163] S. Hawking, Black holes in the Brans-Dicke theory of gravitation, Comm. Math. Phys. 25 (1972) 167-171. doi:10.1007/BF01877518.

[164] D. Sudarsky, J. A. González, Black hole scalar hair in asymptotically anti-de Sitter spacetimes, Phys. Rev. D 67 (2003) 024038.

[165] T. Hertog, G. T. Horowitz, K. Maeda, Negative energy density in Calabi-Yau compactifications, J. High Energy Phys. 2003 (2003) 060.

[166] I. Z. Fisher, Scalar mesostatic field with regard for gravitational effects, Zh. Eksp. Teor. Fiz. 18 (1948) 636, reprinted as eprint arXiv:grqc/9911008. 
[167] O. Bergmann, R. Leipnik, Space-Time Structure of a Static Spherically Symmetric Scalar Field, Phys. Rev. 107 (1957) 1157-1161. doi:10.1103/PhysRev.107.1157.

[168] A. I. Janis, E. T. Newman, J. Winicour, Reality of the Schwarzschild singularity, Phys. Rev. Lett. 20 (1968) 878.

[169] H. A. Buchdahl, Static solutions of the Brans-Dicke equations, Int. J. Theor. Phys. 6 (1972) 407-412. doi:10.1007/BF01258735.

[170] M. Wyman, Static spherically symmetric scalar fields in general relativity, Phys. Rev. D 24 (1981) 839.

[171] A. G. Agnese, M. La Camera, Gravitation without black holes, Lett. Nuovo Cimento 35 (1982) 365-369.

[172] D. D. Dionysiu, Static spherically-symmetric scalar-field theory in general relativity.

[173] A. G. Agnese, M. La Camera, Gravitation without black holes, Phys. Rev. D 31 (1985) 1280.

[174] K. S. Virbhadra, Janis-Newman-Winicour and Wyman solutions are the same, Int. J. Mod. Phys. A 12 (1997) 4831-4835.

[175] M. D. Roberts, Massless scalar static spheres, Astrophys. Space Sci. 200 (1993) 331-335.

[176] J. Formiga, T. Almeida, Wormholes in Wyman's solution, Int. J. Mod. Phys. D 23 (2014) 1450086.

[177] P. Boonserm, T. Ngampitipan, A. Simpson, M. Visser, Exponential metric represents a traversable wormhole, Phys. Rev. D 98 (2018) 084048. arXiv:1805.03781, doi:10.1103/PhysRevD.98.084048.

[178] A. Papapetrou, Eine theorie des gravitationsfeldes mit einer feldfunktion, Zeit. Physik 139 (1954) 518-532.

[179] H. Yilmaz, New approach to general relativity, Phys. Rev. 111 (1958) 1417.

[180] H. Yilmaz, New theory of gravitation, Phys. Rev. Lett. 27 (1971) 1399.

[181] M. A. Makukov, E. G. Mychelkin, Simpler than vacuum: Antiscalar alternatives to black holes, Phys. Rev. D 98 (2018) 064050. 
[182] J. Formiga, Massless scalar field and solar-system experiments, Phys. Rev. D 83 (2011) 087502.

[183] D. Christodoulou, A Mathematical Theory of Gravitational Collapse, Comm. Math. Phys. 109 (1987) 613-647. doi:10.1007/BF01208960.

[184] D. Christodoulou, Examples of naked singularity formation in the gravitational collapse of a scalar field, Ann. Math. 140 (1994) 607653. doi:10.2307/2118619.

[185] M. W. Choptuik, Universality and scaling in gravitational collapse of a massless scalar field, Phys. Rev. Lett. 70 (1993) 9-12. doi:10.1103/PhysRevLett.70.9.

[186] P. R. Brady, Analytic example of critical behaviour in scalar field collapse, Class. Quantum Grav. 11 (1994) 1255-1260. doi:10.1088/0264$9381 / 11 / 5 / 012$.

[187] C. Gundlach, The Choptuik space-time as an eigenvalue problem, Phys. Rev. Lett. 75 (1995) 3214-3217. arXiv:gr-qc/9507054, doi:10.1103/PhysRevLett.75.3214.

[188] C. Gundlach, J. M. Martín-García, Critical phenomena in gravitational collapse, Living Rev. Relativ. 10 (2007) 5.

[189] S. Abe, Stability of a collapsed scalar field and cosmic censorhip, Phys. Rev. D 38 (1988) 1053-1055. doi:10.1103/PhysRevD.38.1053.

[190] D. Garfinkle, G. T. Horowitz, A. Strominger, Charged black holes in string theory, Phys. Rev. D 43 (1991) 3140.

[191] S. Abdolrahimi, A. A. Shoom, Analysis of the Fisher solution, Phys. Rev. D 81 (2010) 024035. arXiv:0911.5380, doi:10.1103/PhysRevD.81.024035.

[192] K. S. Virbhadra, S. Jhingan, P. S. Joshi, Nature of singularity in Einstein massless scalar theory, Int. J. Mod. Phys. D 6 (1997) 357362. arXiv:gr-qc/9512030, doi:10.1142/S0218271897000200.

[193] K. S. Virbhadra, D. Narasimha, S. M. Chitre, Role of the scalar field in gravitational lensing, Astron. Astrophys. 337 (1998) 1-8. arXiv:astroph/9801174. 
[194] K. S. Virbhadra, G. F. R. Ellis, Gravitational lensing by naked singularities, Phys. Rev. D 65 (2002) 103004. doi:10.1103/PhysRevD.65.103004.

[195] K. S. Virbhadra, C. R. Keeton, Time delay and magnification centroid due to gravitational lensing by black holes and naked singularities, Phys. Rev. D 77 (2008) 124014. arXiv:0710.2333, doi:10.1103/PhysRevD.77.124014.

[196] J. D. Bekenstein, Black holes: Classical properties, thermodynamics and heuristic quantization, in: 9th Brazilian School of Cosmology and Gravitation, 1998. arXiv:gr-qc/9808028.

[197] P. O. Mazur, Black hole uniqueness theorems (12 2000). arXiv:hepth/0101012.

[198] K. A. Bronnikov, J. C. Fabris, Regular phantom black holes, Phys. Rev. Lett. 96 (2006) 251101. arXiv:gr-qc/0511109, doi:10.1103/PhysRevLett.96.251101.

[199] H. G. Ellis, Ether flow through a drainhole: a particle model in general relativity, J. Math. Phys. 14 (1973) 104. doi:10.1063/1.1666161.

[200] K. A. Bronnikov, Scalar-tensor theory and scalar charge, Acta Phys. Polon. B 4 (1973) 251-266.

[201] M. Cadoni, E. Franzin, Asymptotically flat black holes sourced by a massless scalar field, Phys. Rev. D 91 (2015) 104011.

[202] A. Anabalón, J. Oliva, Exact hairy black holes and their modification to the universal law of gravitation, Phys. Rev. D 86 (2012) 107501.

[203] A. Anabalón, N. Deruelle, Mechanical stability of asymptotically flat black holes with minimally coupled scalar hair, Phys. Rev. D 88 (2013) 064011.

[204] V. Varela, General relativity, the massless scalar field, and the cosmological constant, Int. J. Theor. Phys. 26 (1987) 91-96.

[205] J. Sultana, Generating time dependent conformally coupled Einsteinscalar solutions, Gen. Relativ. Gravit. 47 (2015) 73.

[206] J. Ibanez, J. Sanz, New exact static solutions to Einstein's equations for spherically symmetric perfect fluid distributions, J. Math. Phys. 23 (1982) 1364-1365. 
[207] J. P. Abreu, P. Crawford, J. P. Mimoso, Exact conformal scalar field cosmologies, Class. Quantum Grav. 11 (1994) 1919-1940. arXiv:grqc/9401024, doi:10.1088/0264-9381/11/8/002.

[208] A. Banijamali, B. Fazlpour, V. Faraoni, Wyman's other scalar field solution, Sultana's generalization, and their Brans-Dicke and $R^{2}$ relatives, Phys. Rev. D 100 (2019) 064017. arXiv:1905.07023, doi:10.1103/PhysRevD.100.064017.

[209] S. Carloni, P. K. S. Dunsby, The $1+1+2$ formalism for scalartensor gravity, Gen. Rel. Grav. 48 (2016) 136. arXiv:1306.2473, doi:10.1007/s10714-016-2131-5.

[210] V. Husain, E. A. Martinez, D. Nuñez, Exact solution for scalar field collapse, Phys. Rev. D 50 (1994) 3783.

[211] O. Fonarev, Exact Einstein scalar field solutions for formation of black holes in a cosmological setting, Class. Quantum Grav. 12 (1995) 1739.

[212] D. Kastor, J. Traschen, Building cosmological frozen stars, Class. Quantum Grav. 34 (2017) 035012.

[213] A. Chamblin, S. Hawking, H. Reall, Brane world black holes, Phys. Rev. D 61 (2000) 065007. arXiv:hep-th/9909205, doi:10.1103/PhysRevD.61.065007.

[214] G. W. Gibbons, K. Maeda, Black holes and membranes in higherdimensional theories with dilaton fields, Nucl. Phys. B 298 (1988) $741-775$.

[215] A. Feinstein, K. Kunze, M. Vazquez-Mozo, Curved dilatonic brane worlds, Phys. Rev. D 64 (2001) 084015.

[216] H. A. Buchdahl, Reciprocal Static Metrics and Scalar Fields in the General Theory of Relativity, Phys. Rev. 115 (1959) 1325-1328. doi:10.1103/PhysRev.115.1325.

[217] K. Tangen, Generating minimally coupled Einstein-scalar field solutions from vacuum solutions with arbitrary cosmological constant (2007). arXiv:0705.4372.

[218] A. I. Janis, D. C. Robinson, J. Winicour, Comments on Einstein scalar solutions, Phys. Rev. 186 (1969) 1729-1731. doi:10.1103/PhysRev.186.1729. 
[219] I. K. Wehus, F. Ravndal, Gravity coupled to a scalar field in extra dimensions, J. Phys. Conf. Ser. 66 (2007) 012024.

[220] H. Maeda, Global structure and physical interpretation of the Fonarev solution for a scalar field with exponential potential (2007). arXiv:0704.2731.

[221] C. Gao, X. Chen, V. Faraoni, Y.-G. Shen, Does the mass of a black hole decrease due to the accretion of phantom energy, Phys. Rev. D 78 (2008) 024008. arXiv:0802.1298, doi:10.1103/PhysRevD.78.024008.

[222] R. Caldwell, A Phantom menace?, Phys. Lett. B 545 (2002) 23-29. arXiv:astro-ph/9908168, doi:10.1016/S0370-2693(02)02589-3.

[223] R. R. Caldwell, M. Kamionkowski, N. N. Weinberg, Phantom energy: dark energy with $w<-1$ causes a cosmic doomsday, Phys. Rev. Lett. 91 (2003) 071301.

[224] S. Nojiri, S. D. Odintsov, Inhomogeneous equation of state of the universe: Phantom era, future singularity, and crossing the phantom barrier, Phys. Rev. D 72 (2005) 023003.

[225] S. Nojiri, S. D. Odintsov, S. Tsujikawa, Properties of singularities in the (phantom) dark energy universe, Phys. Rev. D 71 (2005) 063004.

[226] A. Melchiorri, L. Mersini, C. J. Ödman, M. Trodden, The state of the dark energy equation of state, Phys. Rev. D 68 (2003) 043509.

[227] M. D. Roberts, Scalar field counterexamples to the cosmic censorship hypothesis, Gen. Relativ. Gravit. 21 (1989) 907-939.

[228] R. A. Sussman, Spherically symmetric solutions admitting a spacelike self-similar motion, J. Math. Phys. 32 (1991) 223-230.

[229] P. R. Brady, Does scalar field collapse produce 'zero mass' black holes? (1993). arXiv:gr-qc/9402023.

[230] Y. Oshiro, K. Nakamura, A. Tomimatsu, Critical behavior of black hole formation in a scalar wave collapse, Prog. Theor. Phys. 91 (1994) $1265-1270$.

[231] L. M. Burko, Comment on the Roberts solution for the spherically symmetric Einstein scalar field equations, Gen. Rel. Grav. 29 (1997) 259. arXiv:gr-qc/9608061, doi:10.1023/A:1010200430550. 
[232] S. A. Hayward, An extreme critical spacetime: echoing and black-hole perturbations, Class. Quantum Grav. 17 (2000) 4021.

[233] G. Clement, S. A. Hayward, Comment on 'An extreme critical spacetime: Echoing and black hole perturbations', Class. Quantum Grav. 18 (2001) 4715. arXiv:gr-qc/0108024, doi:10.1088/0264-9381/18/21/401.

[234] R.-G. Cai, Y. Myung, Black holes in the Brans-Dicke-Maxwell theory, Phys. Rev. D 56 (1997) 3466.

[235] A. Wang, H. P. de Oliveira, Critical phenomena of collapsing massless scalar wave packets, Phys. Rev. D 56 (1997) 753.

[236] A. V. Frolov, Continuous self-similarity breaking in critical collapse, Phys. Rev. D 61 (2000) 084006.

[237] H. Maeda, A Simple analytic model of wormhole formations, Phys. Rev. D 79 (2009) 024030. arXiv:0811.2962, doi:10.1103/PhysRevD.79.024030.

[238] H. Maeda, The Roberts-(A) dS spacetime, Class. Quantum Grav. 32 (2015) 135025.

[239] R. M. de Almeida André, Critical Behavior in Gravitational Collapse, MSc thesis, U. Lisbon, 2016.

[240] A. V. Frolov, Perturbations and critical behavior in the self-similar gravitational collapse of a massless scalar field, Phys. Rev. D 56 (1997) 6433-6438. arXiv:gr-qc/9704040, doi:10.1103/PhysRevD.56.6433.

[241] A. V. Frolov, Critical collapse beyond spherical symmetry: General perturbations of the Roberts solution, Phys. Rev. D 59 (1999) 104011. arXiv:gr-qc/9811001, doi:10.1103/PhysRevD.59.104011.

[242] I. I. Gutman, R. M. Bespal'ko, Sbornik Sovrem. Probl. Grav. Tbilissi 1 (1967) 201.

[243] M. D. Roberts, Hybrid imploding scalar and AdS spacetime, Phys. Lett. B 795 (2019) 327-330.

[244] V. Kiselev, Quintessence and black holes, Class. Quantum Grav. 20 (2003) 1187-1198. arXiv:gr-qc/0210040, doi:10.1088/0264$9381 / 20 / 6 / 310$. 
[245] M. Visser, The Kiselev black hole is neither perfect fluid, nor is it quintessence, Class. Quantum Grav. 37 (2020) 045001.

[246] S. S. Bayin, F. I. Cooperstock, V. Faraoni, A singularity-free cosmological model with a conformally coupled scalar field, Astrophys. J. 428 (1994) 439-446. arXiv:astro-ph/9402033, doi:10.1086/174256.

[247] P. Boonserm, T. Ngampitipan, A. Simpson, M. Visser, Decomposition of the total stress energy for the generalized Kiselev black hole, Phys. Rev. D 101 (2020) 024022. arXiv:1910.08008, doi:10.1103/PhysRevD.101.024022.

[248] M. Cvetič, G. W. Gibbons, C. N. Pope, Photon spheres and sonic horizons in black holes from supergravity and other theories, Phys. Rev. D 94 (2016) 106005.

[249] İ. Semiz, Comment on "The Kiselev black hole is neither perfect fluid, nor is it quintessence" (2020). arXiv:2001.06310.

[250] M. Delgaty, K. Lake, Physical acceptability of isolated, static, spherically symmetric, perfect fluid solutions of Einstein's equations, Computer Phys. Comm. 115 (1998) 395-415.

[251] M. Gürses, F. Gürsey, Lorentz Covariant Treatment of the Kerr-Schild Metric, J. Math. Phys. 16 (1975) 2385. doi:10.1063/1.522480.

[252] M. Carrera, D. Giulini, On the generalization of McVittie's model for an inhomogeneity in a cosmological spacetime, Phys. Rev. D 81 (2010) 043521. arXiv:0908.3101, doi:10.1103/PhysRevD.81.043521.

[253] C. Barrabès, P. A. Hogan, Singular Null Hypersurfaces in General Relativity, World Scientific, Singapore, 2003.

[254] A. K. M. Masood-ul Alam, Proof that static stellar models are spherical, Gen. Relativ. Gravit. 39 (2007) 55-85.

[255] H. Pfister, A new and quite general existence proof for static and spherically symmetric perfect fluid stars in general relativity, Class. Quantum Grav. 28 (2011) 075006.

[256] J. R. Oppenheimer, G. M. Volkoff, On massive neutron cores, Phys. Rev. 55 (1939) 374.

[257] R. C. Tolman, Static solutions of Einstein's field equations for spheres of fluid, Phys. Rev. 55 (1939) 364. 
[258] S. Chandrasekhar, An Introduction to the Study of Stellar Structure, Dover, New York, 1958.

[259] M. Wyman, Radially symmetric distributions of matter, Phys. Rev. 75 (1949) 1930.

[260] H. Buchdahl, General-relativistic fluid spheres. III. A static gaseous model, Astrophys. J. 147 (1967) 310.

[261] H. Heintzmann, New exact static solutions of Einstein's field equations, Zeit. Physik 228 (1969) 489-493.

[262] M. R. Finch, J. E. Skea, A realistic stellar model based on an ansatz of Duorah and Ray, Class. Quantum Grav. 6 (1989) 467.

[263] M. Durgapal, A class of new exact solutions in general relativity, J. Phys. A 15 (1982) 2637.

[264] S. Berger, R. Hojman, J. Santamarina, General Exact Solutions of Einstein Equations for Static Perfect Fluids With Spherical Symmetry, J. Math. Phys. 28 (1987) 2949. doi:10.1063/1.527697.

[265] J. Lattimer, M. Prakash, Neutron star structure and the equation of state, Astrophys. J. 550 (2001) 426.

[266] A. D. Rendall, B. G. Schmidt, Existence and properties of spherically symmetric static fluid bodies with a given equation of state, Class. Quantum Grav. 8 (1991) 985.

[267] T. Makino, On spherically symmetric stellar models in general relativity, J. Math. Kyoto Univ. 38 (1998) 55-69.

[268] W. Simon, Criteria for (in)finite extent of static perfect fluids, in: J. Frauendiener, H. Friedrich (Eds.), The Conformal Structure of Space-Time, Springer, New York, 2002, pp. 223-237.

[269] B. Kuchowicz, General relativistic fluid spheres. IV. Differential equations for non-charged spheres of perfect fluid, Acta Phys. Polon. B2 (1971) 657-667.

[270] M. R. Finch, J. E. Skea, A review of the relativistic static fluid sphere, preprint available at http://edradour. symbcomp. uerj. br/pubs. html (1998). 
[271] H. Andréasson, Sharp bounds on $2 m / r$ of general spherically symmetric static objects, J. Diff. Eqs. 245 (2008) 2243-2266.

[272] E. C. Nambo, O. Sarbach, Static spherical perfect fluid stars with finite radius in general relativity: A review (2020). arXiv:2010.02859.

[273] K. Lake, P. Musgrave, The regularity of static spherically cylindrically and plane symmetric spacetimes at the origin, Gen. Relativ. Gravit. 26 (1994) 917-925.

[274] S. L. Liebling, C. Palenzuela, Dynamical boson stars, Living Rev. Relativ. 20 (2017) 5.

[275] L. Andersson, A. Y. Burtscher, On the asymptotic behavior of static perfect fluids, Ann. Inst. H. Poincaré 20 (2019) 813-857.

[276] T. Ramming, G. Rein, Spherically symmetric equilibria for selfgravitating kinetic or fluid models in the nonrelativistic and relativistic case - a simple proof for finite extension, SIAM J. Math. Anal. 45 (2013) 900-914.

[277] P. Boonserm, M. Visser, Buchdahl-like transformations for perfect fluid spheres, Int. J. Mod. Phys. D 17 (2008) 135-163. arXiv:0707.0146, doi:10.1142/S0218271808011912.

[278] K. Lake, All static spherically symmetric perfect-fluid solutions of Einstein's equations, Phys. Rev. D 67 (2003) 104015.

[279] S. Rahman, M. Visser, Spacetime geometry of static fluid spheres, Class. Quantum Grav. 19 (2002) 935.

[280] D. Martin, M. Visser, Algorithmic construction of static perfect fluid spheres, Phys. Rev. D 69 (2004) 104028.

[281] P. Boonserm, M. Visser, S. Weinfurtner, Generating perfect fluid spheres in general relativity, Phys. Rev. D 71 (2005) 124037. arXiv:grqc/0503007, doi:10.1103/PhysRevD.71.124037.

[282] P. Boonserm, M. Visser, S. Weinfurtner, Solution generating theorems for perfect fluid spheres, J. Phys. Conf. Ser. 68 (2007) 012055. arXiv:gr-qc/0609088, doi:10.1088/1742-6596/68/1/012055. 
[283] P. Boonserm, M. Visser, S. Weinfurtner, Solution generating theorems: Perfect fluid spheres and the TOV equation, in: 11th Marcel Grossmann Meeting on General Relativity, 2006, pp. 2285-2287. arXiv:gr-qc/0609099.

[284] İ. Semiz, The general static spherical perfect fluid solution with EoS parameter $w=-1 / 5$ (2020). arXiv:2007.08166.

[285] M. Ishak, K. Lake, An online interactive geometric database: Including exact solutions of Einstein's field equations, Class. Quantum Grav. 19 (2002) 505-514. arXiv:gr-qc/0111008, doi:10.1088/0264$9381 / 19 / 3 / 306$.

[286] J. R. Oppenheimer, H. Snyder, On continued gravitational contraction, Phys. Rev. 56 (1939) 455.

[287] G. C. McVittie, An example of gravitational collapse in general relativity, Astrophys. J. 143 (1966) 682.

[288] H. Bondi, Spherically symmetrical models in general relativity, Mon. Not. Roy. Astron. Soc. 107 (1947) 410-425.

[289] R. Mansouri, On the non-existence of time-dependent fluid spheres in general relativity obeying an equation of state, Ann. Inst. H. Poincaré 27 (1977) 175-183.

[290] B. Mashhoon, M. H. Partovi, On the gravitational motion of a fluid obeying an equation of state, Ann. Phys. (N.Y.) 130 (1980) 99-138.

[291] E. Glass, Shear-free gravitational collapse, J. Math. Phys. 20 (1979) $1508-1513$.

[292] J. Smoller, B. Temple, Shock-wave solutions in closed form and the Oppenheimer-Snyder limit in general reality, Siam J. Appl. Math. 58 (1998) 15-33.

[293] P. C. Vaidya, Nonstatic analogs of Schwarzschild's interior solution in general relativity, Phys. Rev. 174 (1968) 1615.

[294] I. H. Thompson, G. J. Whitrow, Time-dependent internal solutions for spherically symmetrical bodies in general relativity: I. Adiabatic collapse, Mon. Not. Roy. Astron. Soc. 136 (1967) 207-217. 
[295] I. H. Thompson, G. J. Whitrow, Time-dependent internal solutions for spherically symmetrical bodies in general relativity: II. Adiabatic radial motions of uniformly dense spheres, Mon. Not. Roy. Astron. Soc. 139 (1968) 499-513.

[296] P. Boonserm, T. Ngampitipan, M. Visser, Mimicking static anisotropic fluid spheres in general relativity, Int. J. Mod. Phys. D 25 (2015) 1650019. arXiv:1501.07044, doi:10.1142/S021827181650019X.

[297] B. C. Nolan, A point mass in an isotropic universe: II. Global properties, Class. Quantum Grav. 16 (1999) 1227.

[298] N. Afshordi, D. J. H. Chung, M. Doran, G. Geshnizjani, Cuscuton Cosmology: Dark Energy meets Modified Gravity, Phys. Rev. D 75 (2007) 123509. arXiv:astro-ph/0702002, doi:10.1103/PhysRevD.75.123509.

[299] G. Gibbons, C. Warnick, M. Werner, Light-bending in Schwarzschildde-Sitter: Projective geometry of the optical metric, Class. Quantum Grav. 25 (2008) 245009. arXiv:0808.3074, doi:10.1088/0264$9381 / 25 / 24 / 245009$.

[300] N. Afshordi, Cuscuton and low energy limit of Horava-Lifshitz gravity, Phys. Rev. D 80 (2009) 081502. arXiv:0907.5201, doi:10.1103/PhysRevD.80.081502.

[301] N. Kaloper, M. Kleban, D. Martin, McVittie's legacy: Black holes in an expanding universe, Phys. Rev. D 81 (2010) 104044.

[302] J. P. Mimoso, M. Le Delliou, F. C. Mena, Separating expansion from contraction in spherically symmetric models with a perfect fluid: Generalization of the Tolman-Oppenheimer-Volkoff condition and application to models with a cosmological constant, Phys. Rev. D 81 (2010) 123514 .

[303] H. Arakida, Application of time transfer function to McVittie spacetime: gravitational time delay and secular increase in astronomical unit, Gen. Relativ. Gravit. 43 (2011) 2127-2139.

[304] M. Le Delliou, F. C. Mena, J. P. Mimoso, The role of shell crossing on the existence and stability of trapped matter shells in spherical inhomogeneous Lambda-CDM models, Phys. Rev. D 83 (2011) 103528. arXiv:1103.0976, doi:10.1103/PhysRevD.83.103528. 
[305] K. Lake, M. Abdelqader, More on McVittie's legacy: A Schwarzschildde Sitter black and white hole embedded in an asymptotically $\Lambda \mathrm{CDM}$ cosmology, Phys. Rev. D 84 (2011) 044045.

[306] D. C. Guariento, M. Fontanini, A. M. da Silva, E. Abdalla, Realistic fluids as source for dynamically accreting black holes in a cosmological background, Phys. Rev. D 86 (2012) 124020.

[307] V. Faraoni, A. F. Zambrano Moreno, R. Nandra, Making sense of the bizarre behavior of horizons in the McVittie spacetime, Phys. Rev. D 85 (2012) 083526.

[308] R. Nandra, A. N. Lasenby, M. P. Hobson, The effect of a massive object on an expanding universe, Mon. Not. Roy. Astron. Soc. 422 (2012) 2931-2944. arXiv:1104.4447, doi:10.1111/j.1365-2966.2012.20618.x.

[309] R. Nandra, A. N. Lasenby, M. P. Hobson, The effect of an expanding universe on massive objects, Mon. Not. Roy. Astron. Soc. 422 (2012) 2945-2959. arXiv:1104.4458, doi:10.1111/j.1365-2966.2012.20617.x.

[310] V. Faraoni, A. F. Zambrano Moreno, Are quantization rules for horizon areas universal?, Phys. Rev. D 88 (2013) 044011.

[311] M. Le Delliou, J. P. Mimoso, F. C. Mena, M. Fontanini, D. C. Guariento, E. Abdalla, Separating expansion and collapse in general fluid models with heat flux, Phys. Rev. D 88 (2013) 027301. arXiv:1305.3475, doi:10.1103/PhysRevD.88.027301.

[312] A. M. da Silva, M. Fontanini, D. C. Guariento, How the expansion of the universe determines the causal structure of McVittie spacetimes, Phys. Rev. D 87 (2013) 064030.

[313] J. P. Mimoso, M. Le Delliou, F. C. Mena, Local conditions separating expansion from collapse in spherically symmetric models with anisotropic pressures, Phys. Rev. D 88 (2013) 043501.

[314] V. Faraoni, A. F. Zambrano Moreno, A. Prain, Charged McVittie spacetime, Phys. Rev. D 89 (2014) 103514.

[315] N. Afshordi, M. Fontanini, D. C. Guariento, Horndeski meets McVittie: A scalar field theory for accretion onto cosmological black holes, Phys. Rev. D 90 (2014) 084012. doi:10.1103/PhysRevD.90.084012. 
[316] E. Abdalla, N. Afshordi, M. Fontanini, D. C. Guariento, E. Papantonopoulos, Cosmological black holes from self-gravitating fields, Phys. Rev. D 89 (2014) 104018. arXiv:1312.3682, doi:10.1103/PhysRevD.89.104018.

[317] A. Maciel, M. Le Delliou, J. P. Mimoso, Dual null formalism for the collapse of fluids in a cosmological background, Phys. Rev. D 92 (2015) 083525 .

[318] A. Maciel, D. C. Guariento, C. Molina, Cosmological black holes and white holes with time-dependent mass, Phys. Rev. D 91 (2015) 084043.

[319] M. M. C. Mello, A. Maciel, V. T. Zanchin, Evolving black holes from conformal transformations of static solutions, Phys. Rev. D 95 (2017) 084031.

[320] O. F. Piattella, Erratum: Lensing in the McVittie metric [Phys. Rev. D 93, 024020 (2016)], Phys. Rev. D 93 (2016) 129901.

[321] M. E. Aghili, B. Bolen, L. Bombelli, Effect of accelerated global expansion on the bending of light, Gen. Relativ. Gravit. 49 (2017) 10.

[322] V. Faraoni, M. Lapierre-Léonard, Beyond lensing by the cosmological constant, Phys. Rev. D 95 (2017) 023509.

[323] Y. P. Shah, P. C. Vaidya, Gravitational field of a charged particle embedded in a homogeneous universe, Tensor 19 (1968) 191.

[324] B. Mashhoon, M. H. Partovi, Gravitational collapse of a charged fluid sphere, Phys. Rev. D 20 (1979) 2455.

[325] V. Faraoni, A. Jacques, Cosmological expansion and local physics, Phys. Rev. D 76 (2007) 063510.

[326] Z.-H. Li, A. Wang, Existence of black holes in FriedmannRobertson-Walker universe dominated by dark energy, Mod. Phys. Lett. A 22 (2007) 1663-1676. arXiv:astro-ph/0607554, doi:10.1142/S0217732307024048.

[327] G. C. McVittie, The mass-particle in an expanding universe, Mon. Not. Roy. Astron. Soc. 93 (1933) 325-339. doi:10.1093/mnras/93.5.325.

[328] R. P. A. C. Newman, G. C. McVittie, A point-particle model universe, Gen. Relativ. Gravit. 14 (1982) 591-608. 
[329] M. Ferraris, M. Francaviglia, A. Spallicci, Associated radius, energy and pressure of McVittie's metric in its astrophysical application, Nuovo Cimento B 111 (1996) 1031-1036.

[330] B. C. Nolan, Sources for McVittie's mass particle in an expanding universe, J. Math. Phys. 34 (1993) 178-185.

[331] B. C. Nolan, A point mass in an isotropic universe: Existence, uniqueness, and basic properties, Phys. Rev. D 58 (1998) 064006.

[332] P. Landry, M. Abdelqader, K. Lake, McVittie solution with a negative cosmological constant, Phys. Rev. D 86 (2012) 084002.

[333] M. Anderson, Horizons, singularities and causal structure of the generalized McVittie space-times, J. Phys. Conf. Ser. 283 (2011) 012001.

[334] A. K. Raychaudhuri, Theoretical Cosmology, Cambridge University Press, Cambridge, UK, 1979.

[335] P. Kustaanheimo, B. Qvist, Comm. Phys.-Math. Soc. Sci. Fennica 13 (1948) 1-11, reprinted in Gen. Relativ. Gravit. 30, 659 (1998).

[336] H. Gomes, S. Gryb, T. Koslowski, Einstein gravity as a 3D conformally invariant theory, Class. Quantum Grav. 28 (2011) 045005.

[337] C. Bejarano, R. Ferraro, M. J. Guzmán, McVittie solution in $f(T)$ gravity, Eur. Phys. J. C 77 (2017) 825. arXiv:1707.06637, doi:10.1140/epjc/s10052-017-5394-4.

[338] R. A. Sussman, Conformal structure of a Schwarzschild black hole immersed in a Friedman universe, Gen. Relativ. Gravit. 17 (1985) $251-291$.

[339] M. L. McClure, C. C. Dyer, Asymptotically Einstein-de Sitter cosmological black holes and the problem of energy conditions, Class. Quantum Grav. 23 (2006) 1971-1987. doi:10.1088/0264-9381/23/6/008.

[340] M. L. McClure, C. C. Dyer, Matching radiation-dominated and matter-dominated Einstein-de Sitter universes and an application for primordial black holes in evolving cosmological backgrounds, Gen. Rel. Grav. 38 (2006) 1347-1354. doi:10.1007/s10714-006-0321-2.

[341] C. J. Gao, S. N. Zhang, Reissner-Nordström metric in the FriedmanRobertson-Walker universe, Phys. Lett. B 595 (2004) 28-35. 
[342] C. J. Gao, S. N. Zhang, Higher dimensional Reissner-NordstromFRW metric, Gen. Rel. Grav. 38 (2006) 23-32. arXiv:gr-qc/0411040, doi:10.1007/s10714-005-0207-8.

[343] V. Faraoni, C. Gao, X. Chen, Y.-G. Shen, What is the fate of a black hole embedded in an expanding universe?, Phys. Lett. B 671 (2009) $7-9$.

[344] S. N. G. Thakurta, Kerr metric in an expanding universe, Indian J. Phys. B 55 (1981) 304-310.

[345] T. Clifton, D. F. Mota, J. D. Barrow, Inhomogeneous gravity, Mon. Not. Roy. Astron. Soc. 358 (2005) 601-613.

[346] A. Einstein, E. G. Straus, The influence of the expansion of space on the gravitation fields surrounding the individual stars, Rev. Mod. Phys. 17 (1945) 120.

[347] A. Einstein, E. G. Straus, Corrections and additional remarks to our paper: The influence of the expansion of space on the gravitation fields surrounding the individual stars, Rev. Mod. Phys. 18 (1946) 148.

[348] J. M. M. Senovilla, R. Vera, Impossibility of the cylindrically symmetric Einstein-Straus model, Phys. Rev. Lett. 78 (1997) 2284.

[349] M. Mars, On the uniqueness of the Einstein-Straus model, Class. Quantum Grav. 18 (2001) 3645.

[350] F. C. Mena, R. Tavakol, R. Vera, Generalization of the Einstein-Straus model to anisotropic settings, Phys. Rev. D 66 (2002) 044004.

[351] M. Carrera, D. Giulini, Influence of global cosmological expansion on local dynamics and kinematics, Rev. Mod. Phys. 82 (2010) 169.

[352] V. Marra, E. W. Kolb, S. Matarrese, A. Riotto, Cosmological observables in a Swiss-cheese universe, Phys. Rev. D 76 (2007) 123004.

[353] T. Biswas, A. Notari, Swiss-Cheese Inhomogeneous Cosmology and the Dark Energy Problem, J. Cosmol. Astropart. Phys. 06 (2008) 021. arXiv:astro-ph/0702555, doi:10.1088/1475-7516/2008/06/021.

[354] G. Lemaître, The expanding universe, Ann. Soc. Sci. Bruxelles A 53 (1933) 51, reprinted in Gen. Relativ. Gravit. 29 (1997) 641. 
[355] R. C. Tolman, Effect of inhomogeneity on cosmological models, Proc. Natl. Acad. Sci. U.S.A. 20 (1934) 169.

[356] J. Sultana, C. C. Dyer, Cosmological black holes: A black hole in the Einstein-de Sitter universe, Gen. Relativ. Gravit. 37 (2005) 1347-1370.

[357] M. L. McClure, K. Anderson, K. Bardahl, Nonisolated dynamic black holes and white holes, Phys. Rev. D 77 (2008) 104008.

[358] H. Culetu, On the time dependent Schwarzschild-de Sitter spacetime (2012). arXiv:1201.3769.

[359] D. D. McNutt, D. N. Page, Scalar polynomial curvature invariant vanishing on the event horizon of any black hole metric conformal to a static spherical metric, Phys. Rev. D 95 (2017) 084044.

[360] H. Saida, T. Harada, H. Maeda, Black hole evaporation in an expanding universe, Class. Quant. Grav. 24 (2007) 4711-4732. arXiv:0705.4012, doi:10.1088/0264-9381/24/18/011.

[361] B. Carr, T. Harada, H. Maeda, Can a primordial black hole or wormhole grow as fast as the universe?, Class. Quantum Grav. 27 (2010) 183101. arXiv:1003.3324, doi:10.1088/0264-9381/27/18/183101.

[362] S. Chakrabarti, N. Banerjee, Scalar field collapse in a conformally flat spacetime, Eur. Phys. J. C 77 (2017) 166. arXiv:1609.01868, doi:10.1140/epjc/s10052-017-4740-x.

[363] J. Firouzjaee, R. Mansouri, Asymptotically FRW black holes, Gen. Relativ. Gravit. 42 (2010) 2431-2452.

[364] D. Kastor, J. Traschen, Cosmological multi-black-hole solutions, Phys. Rev. D 47 (1993) 5370.

[365] C.-Y. Sun, Naked singularity in the Sultana-Dyer space-time (2009). arXiv:0906.3783.

[366] B. Carter, Axisymmetric Black Hole Has Only Two Degrees of Freedom, Phys. Rev. Lett. 26 (1971) 331-333. doi:10.1103/PhysRevLett.26.331.

[367] R. M. Wald, Final states of gravitational collapse, Phys. Rev. Lett. 26 (1971) 1653. 
[368] C. Charmousis, in: E. Papantonopoulos (Ed.), Physics of Black Holes, Proceedings of the Fourth Aegean School on Black Holes, Lesvos, Greece, Springer, New York, 2009, pp. 299-343.

[369] T. P. Sotiriou, in: E. Papantonopoulos (Ed.), Modifications of Einstein's Theory of Gravity at Large Distances, Proceedings of the Seventh Aegean Summer School, Parikia, Greece, Springer, New York, 2015, pp. 3-33.

[370] J. Callan, Curtis G., E. Martinec, M. Perry, D. Friedan, Strings in Background Fields, Nucl. Phys. B 262 (1985) 593-609. doi:10.1016/0550-3213(85)90506-1.

[371] E. S. Fradkin, A. A. Tseytlin, Quantum string theory effective action, Nucl. Phys. B 261 (1985) 1-27.

[372] P. Higgs, Quadratic Lagrangians and general relativity, Nuovo Cimento 11 (1959) 816-820.

[373] V. Faraoni, The $\omega \rightarrow \infty$ limit of Brans-Dicke theory, Phys. Lett. A 245 (1998) 26-30.

[374] V. Faraoni, Illusions of general relativity in Brans-Dicke gravity, Phys. Rev. D 59 (1999) 084021.

[375] C. Santos, Static black holes in scalar tensor gravity (2000). arXiv:grqc/0009054.

[376] T. P. Sotiriou, V. Faraoni, Black holes in scalar-tensor gravity, Phys. Rev. Lett. 108 (2012) 081103.

[377] S. Bhattacharya, K. F. Dialektopoulos, A. E. Romano, T. N. Tomaras, Brans-Dicke Theory with $\Lambda>0$ : Black Holes and Large Scale Structures, Phys. Rev. Lett. 115 (2015) 181104. arXiv:1505.02375, doi:10.1103/PhysRevLett.115.181104.

[378] V. Faraoni, Jebsen-Birkhoff theorem in alternative gravity, Phys. Rev. D 81 (2010) 044002.

[379] M. A. Scheel, S. L. Shapiro, S. A. Teukolsky, Collapse to black holes in Brans-Dicke theory. I Horizon boundary conditions for dynamical spacetimes, Phys. Rev. D 51 (1995) 4208. 
[380] M. A. Scheel, S. L. Shapiro, S. A. Teukolsky, Collapse to black holes in Brans-Dicke theory. 2. Comparison with general relativity, Phys. Rev. D 51 (1995) 4236-4249. arXiv:gr-qc/9411026, doi:10.1103/PhysRevD.51.4236.

[381] J. Kerimo, D. Kalligas, Gravitational collapse of collisionless matter in scalar-tensor theories: Scalar waves and black hole formation, Phys. Rev. D 58 (1998) 104002.

[382] J. Kerimo, Dynamical black holes in scalar-tensor theories, Phys. Rev. D 62 (2000) 104005.

[383] T. P. Sotiriou, S.-Y. Zhou, Black hole hair in generalized scalartensor gravity, Phys. Rev. Lett. 112 (2014) 251102. arXiv:1312.3622, doi:10.1103/PhysRevLett.112.251102.

[384] E. Babichev, C. Charmousis, A. Lehébel, Black holes and stars in Horndeski theory, Class. Quant. Grav. 33 (15) (2016) 154002. arXiv:1604.06402, doi:10.1088/0264-9381/33/15/154002.

[385] D. R. K. Reddy, On Birkhoff's theorem in scalar-tensor theory of gravitation, J. Phys. A 6 (1973) 1867.

[386] K. Krori, D. Nandy, Birkhoff's theorem and scalar-tensor theories of gravitation, J. Phys. A 10 (1977) 993.

[387] V. Faraoni, F. Hammad, A. M. Cardini, T. Gobeil, Revisiting the analogue of the Jebsen-Birkhoff theorem in Brans-Dicke gravity, Phys. Rev. D 97 (2018) 084033.

[388] A. G. Agnese, M. La Camera, Wormholes in the Brans-Dicke theory of gravitation, Phys. Rev. D 51 (1995) 2011.

[389] M. Campanelli, C. Lousto, Are black holes in Brans-Dicke theory precisely the same as in general relativity?, Int. J. Mod. Phys. D 2 (1993) 451-462.

[390] C. Lousto, M. Campanelli, in: E. Gunzig, P. Nardone (Eds.), The Origin of Structure in the Universe, Pont d'Oye, Belgium, 1992, Kluwer Academic, Dordrecht, 1993, p. 123.

[391] L. Vanzo, S. Zerbini, V. Faraoni, Campanelli-Lousto and veiled spacetimes, Phys. Rev. D 86 (2012) 084031. 
[392] A. Bhadra, K. Sarkar, On static spherically symmetric solutions of the vacuum Brans-Dicke theory, Gen. Relat. Gravit. 37 (2005) 2189-2199.

[393] K. A. Bronnikov, C. P. Constantinidis, R. L. Evangelista, J. C. Fabris, Electrically charged cold black holes in scalar-tensor theories, Int. J. Mod. Phys. D 8 (1999) 481-505.

[394] K. A. Bronnikov, G. Clement, C. P. Constantinidis, J. C. Fabris, Structure and stability of cold scalar-tensor black holes, Phys. Lett. A 243 (1998) 121-127. arXiv:gr-qc/9801050, doi:10.1016/S03759601(98)00133-9.

[395] N. Van den Bergh, General solutions for a static isotropic metric in the Brans-Dicke gravitational theory, Gen. Relativ. Gravit. 12 (1980) 863-869.

[396] C. Brans, Mach's Principle and a Relativistic Theory of Gravitation. II, Phys. Rev. 125 (1962) 2194-2201. doi:10.1103/PhysRev.125.2194.

[397] A. Bhadra, K. K. Nandi, Brans type II-IV solutions in the Einstein frame and physical interpretation of constants in the solutions, Mod. Phys. Lett. A 16 (2001) 2079-2087. doi:10.1142/S0217732301005539.

[398] V. Faraoni, F. Hammad, S. D. Belknap-Keet, Revisiting the Brans solutions of scalar-tensor gravity, Phys. Rev. D 94 (2016) 104019.

[399] F. He, S.-W. Kim, New Brans-Dicke wormholes, Phys. Rev. D 65 (2002) 084022.

[400] A. Bhadra, I. Simaciu, K. K. Nandi, Y.-Z. Zhang, Comment on 'New Brans-Dicke wormholes', Phys. Rev. D 71 (2005) 128501. arXiv:grqc/0406014, doi:10.1103/PhysRevD.71.128501.

[401] K. A. Bronnikov, M. V. Skvortsova, A. A. Starobinsky, Notes on wormhole existence in scalar-tensor and $F(R)$ gravity, Grav. Cosmol. 16 (2010) 216-222. arXiv:1005.3262, doi:10.1134/S0202289310030047.

[402] V. Faraoni, V. Vitagliano, T. P. Sotiriou, S. Liberati, Dynamical apparent horizons in inhomogeneous Brans-Dicke universes, Phys. Rev. D 86 (2012) 064040.

[403] V. Faraoni, A. F. Zambrano Moreno, Interpreting the conformal cousin of the Husain-Martinez-Nuñez spacetime, Phys. Rev. D 86 (2012) 084044 . 
[404] V. Faraoni, S. D. Belknap-Keet, New inhomogeneous universes in scalar-tensor and $f(R)$ gravity, Phys. Rev. D 96 (2017) 044040.

[405] A. Linde, Particle Physics and Inflationary Cosmology, Harwood Academic, Chur, Switzerland, 1990.

[406] A. R. Liddle, D. H. Lyth, Cosmological Inflation and Large-Scale Structure, Cambridge University Press, Cambridge, UK, 2000.

[407] P. J. E. Peebles, B. Ratra, The cosmological constant and dark energy, Rev. Mod. Phys. 75 (2003) 559.

[408] D. Wands, E. J. Copeland, A. R. Liddle, Exponential potentials, scaling solutions and inflation, Ann. N.Y. Acad. Sci. 688 (1993) 647.

[409] V. Faraoni, D. K. Çiftci, S. D. Belknap-Keet, Symmetry of BransDicke gravity as a novel solution-generating technique, Phys. Rev. D 97 (2018) 064004.

[410] N. Chernikov, E. Tagirov, Quantum theory of scalar field in de Sitter space-time, Ann. Inst. H. Poincaré 9 (1968) 109-141.

[411] C. G. J. Callan, S. Coleman, R. Jackiw, A new improved energymomentum tensor, Ann. Phys. (N.Y.) 59 (1970) 42-73.

[412] N. D. Birrell, P. C. W. Davies, Quantum Fields in Curved Space, Cambridge University Press, Cambridge, UK, 1984.

[413] N. D. Birrell, P. C. W. Davies, Conformal Symmetry Breaking and Cosmological Particle Creation in $\lambda \phi^{4}$ Theory, Phys. Rev. D 22 (1980) 322. doi:10.1103/PhysRevD.22.322.

[414] B. L. Nelson, P. Panangaden, Scaling behavior of interacting quantum fields in curved spacetime, Phys. Rev. D 25 (1982) 1019.

[415] L. Ford, D. J. Toms, Dynamical symmetry breaking due to radiative corrections in cosmology, Phys. Rev. D 25 (1982) 1510.

[416] L. Parker, D. J. Toms, Renormalization-group analysis of grand unified theories in curved spacetime, Phys. Rev. D 29 (1984) 1584.

[417] L. Ford, Gravitational particle creation and inflation, Phys. Rev. D 35 (1987) 2955. 
[418] S. Sonego, V. Faraoni, Coupling to the curvature for a scalar field from the equivalence principle, Class. Quantum Grav. 10 (1993) 1185.

[419] G. F. R. Ellis, D. W. Sciama, Global and non-global problems in cosmology, in: L. O'Raifeartaigh (Ed.), General Relativity, Papers in Honour of J.L. Synge, Clarendon Press: Oxford, UK, 1972.

[420] B. S. DeWitt, R. W. Brehme, Radiation damping in a gravitational field, Ann. Phys. (N.Y.) 9 (1960) 220-259. doi:10.1016/00034916(60)90030-0.

[421] G. G. Friedlander, The Wave Equation On A Curved Space-Time, Cambridge University Press, Cambridge, UK, 1975.

[422] V. Faraoni, E. Gunzig, Tales of tails in cosmology, Int. J. Mod. Phys. D 8 (1999) 177-188. arXiv:astro-ph/9902262, doi:10.1142/S021827189900016X.

[423] T. Okamura, in: Y. Cho, C. Lee, S.-W. Kim (Eds.), Proceedings of the Asia-Pacific Conference on Gravitation and Cosmology, Seoul, Korea 1996, World Scientific, Singapore, 1998.

[424] X.-l. Chen, R. J. Scherrer, G. Steigman, Extended quintessence and the primordial helium abundance, Phys. Rev. D 63 (2001) 123504. arXiv:astro-ph/0011531, doi:10.1103/PhysRevD.63.123504.

[425] L. F. Abbott, Gravitational Effects on the SU(5) Breaking Phase Transition for a Coleman-Weinberg Potential, Nucl. Phys. B 185 (1981) 233-238. doi:10.1016/0550-3213(81)90374-6.

[426] F. Lucchin, S. Matarrese, M. D. Pollock, Inflation with a nonminimally coupled scalar field, Phys. Lett. B 167 (1986) 163-168.

[427] T. Futamase, K. Maeda, Chaotic inflationary scenario of the universe with a nonminimally coupled "inflaton" field, Phys. Rev. D 39 (1989) 399.

[428] T. Futamase, T. Rothman, R. Matzner, Behavior of chaotic inflation in anisotropic cosmologies with nonminimal coupling, Phys. Rev. D 39 (1989) 405.

[429] V. Faraoni, Nonminimal coupling of the scalar field and inflation, Phys. Rev. D 53 (1996) 6813. 
[430] M. B. Voloshin, A. D. Dolgov, On gravitational interaction of the Goldtsone bosons, Sov. J. Nucl. Phys. 35 (1982) 120-121.

[431] C. T. Hill, D. S. Salopek, Calculable nonminimal coupling of composite scalar bosons to gravity, Ann. Phys. (N.Y.) 213 (1992) 21-30.

[432] M. Reuter, Nonminimal gravitational coupling of scalar bound states, Phys. Rev. D 49 (1994) 6379.

[433] Y. Hosotani, Stability of Scalar Fields in Curved Space, Phys. Rev. D 32 (1985) 1949. doi:10.1103/PhysRevD.32.1949.

[434] I. L. Buchbinder, S. D. Odintsov, Asymptotical properties of nonAbelian gauge theories in external gravitational fields, Sov. J. Nucl. Phys. 40 (1984) 848.

[435] I. L. Buchbinder, S. D. Odintsov, Asymptotical conformal invariance in curved space-time, Lett. Nuovo Cim. 42 (1985) 379-381. doi:10.1007/BF02747058.

[436] E. Elizalde, S. D. Odintsov, Renormalization-group improved effective potential for finite grand unified theories in curved spacetime, Phys. Lett. B 333 (1994) 331-336.

[437] I. L. Buchbinder, S. D. Odintsov, I. Shapiro, Effective Action in Quantum Gravity, IOP Publishing, Bristol, 1992.

[438] I. L. Buchbinder, S. D. Odintsov, I. M. Lichtzier, The behaviour of effective coupling constants in 'finite' grand unification theories in curved spacetime, Class. Quantum Grav. 6 (1989) 605.

[439] A. Bonanno, Coarse graining and renormalization group in the Einstein universe, Phys. Rev. D 52 (1995) 969-980. arXiv:gr-qc/9505051, doi:10.1103/PhysRevD.52.969.

[440] T. Futamase, M. Tanaka, Chaotic inflation with a running nonminimal coupling, Phys. Rev. D 60 (1999) 063511.

[441] N. M. Bocharova, K. A. Bronnikov, V. N. Melnikov, An exact solution of the system of Einstein equations and mass-free scalar field, Vestn. Mosk. Univ. Fiz. Astron 6 (1970) 706.

[442] J. D. Bekenstein, Exact solutions of Einstein-conformal scalar equations, Ann. Phys. (N.Y.) 82 (1974) 535-547. 
[443] J. Frøyland, Static, spherically symmetric scalar fields with a traceless energy-momentum tensor in general relativity, Phys. Rev. D 25 (1982) 1470 .

[444] J. D. Bekenstein, Black holes with scalar charge, Ann. Phys. (N.Y.) 91 (1975) 75-82.

[445] B. C. Xanthopoulos, T. Zannias, The uniqueness of the Bekenstein black hole, J. Math. Phys. 32 (1991) 1875-1880.

[446] B. C. Xanthopoulos, T. E. Dialynas, Einstein gravity coupled to a massless conformal scalar field in arbitrary space-time dimensions, J. Math. Phys. 33 (1992) 1463-1471.

[447] B. C. Xanthopoulos, T. Zannias, Einstein gravity coupled to a massless scalar field in arbitrary spacetime dimensions, Phys. Rev. D 40 (1989) 2564 .

[448] C. Klimcík, Search for the conformal scalar hair at arbitrary D, J. Math. Phys. 34 (1993) 1914-1926. doi:10.1063/1.530146.

[449] D. Sudarsky, T. Zannias, Spherical black holes cannot support scalar hair, Phys. Rev. D 58 (1998) 087502.

[450] O. B. Zaslavskii, Thermodynamics of black holes with an infinite effective area of a horizon, Class. Quantum Grav. 19 (2002) 3783-3798. arXiv:gr-qc/0206018, doi:10.1088/0264-9381/19/14/317.

[451] K. A. Bronnikov, Y. N. Kireev, Instability of Black Holes with Scalar Charge, Phys. Lett. A 67 (1978) 95-96. doi:10.1016/03759601(78)90030-0.

[452] D.-C. Zou, Y. S. Myung, Scalar hairy black holes in Einstein-Maxwellconformally coupled scalar theory, Phys. Lett. B 803 (2020) 135332.

[453] Y. Tomikawa, T. Shiromizu, K. Izumi, On the uniqueness of the static black hole with conformal scalar hair, Prog. Theor. Exp. Phys. 2017 (2017).

[454] Y. Tomikawa, T. Shiromizu, K. Izumi, On uniqueness of static spacetimes with non-trivial conformal scalar field, Class. Quantum Grav. 34 (2017) 155004. 
[455] M. Astorino, Stationary axisymmetric spacetimes with a conformally coupled scalar field, Phys. Rev. D 91 (2015) 064066. arXiv:1412.3539, doi:10.1103/PhysRevD.91.064066.

[456] S. Bhattacharya, H. Maeda, Can a black hole with conformal scalar hair rotate?, Phys. Rev. D 89 (2014) 087501. arXiv:1311.0087, doi:10.1103/PhysRevD.89.087501.

[457] C. Martinez, R. Troncoso, J. Zanelli, De Sitter black hole with a conformally coupled scalar field in four-dimensions, Phys. Rev. D 67 (2003) 024008. arXiv:hep-th/0205319, doi:10.1103/PhysRevD.67.024008.

[458] K. S. Virbhadra, J. C. Parikh, A conformal scalar dyon black hole solution, Phys. Lett. B 331 (1994) 302-304.

[459] C. Martinez, J. P. Staforelli, R. Troncoso, Topological black holes dressed with a conformally coupled scalar field and electric charge, Phys. Rev. D 74 (2006) 044028. arXiv:hep-th/0512022, doi:10.1103/PhysRevD.74.044028.

[460] A.-M. Barlow, D. Doherty, E. Winstanley, Thermodynamics of de Sitter black holes with a conformally coupled scalar field, Phys. Rev. D 72 (2005) 024008. arXiv:gr-qc/0504087, doi:10.1103/PhysRevD.72.024008.

[461] C. Charmousis, T. Kolyvaris, E. Papantonopoulos, Charged C-metric with conformally coupled scalar field, Class. Quantum Grav. 26 (2009) 175012. arXiv:0906.5568, doi:10.1088/0264-9381/26/17/175012.

[462] J. F. Plebański, M. Demiański, Rotating, charged, and uniformly accelerating mass in general relativity, Ann. Phys. (N.Y.) 98 (1976) 98127.

[463] J. B. Griffiths, J. Podolskỳ, A new look at the Plebański-Demiański family of solutions, Int. J. Mod. Phys. D 15 (2006) 335-369.

[464] A. Anabalón, H. Maeda, New Charged Black Holes with Conformal Scalar Hair, Phys. Rev. D 81 (2010) 041501. arXiv:0907.0219, doi:10.1103/PhysRevD.81.041501.

[465] I. Bengtsson, S. Holst, E. Jakobsson, Classics Illustrated: Limits of Spacetimes, Class. Quant. Grav. 31 (2014) 205008. arXiv:1406.4326, doi:10.1088/0264-9381/31/20/205008. 
[466] D. A. Carranza, A. E. Hursit, J. A. Valiente Kroon, Conformal wave equations for the Einstein-tracefree matter system, Gen. Rel. Grav. 51 (2019) 88. arXiv:1902.01816, doi:10.1007/s10714-019-2567-5.

[467] P. D. Prester, Field redefinitions, Weyl invariance and the nature of mavericks, Class. Quantum Grav. 31 (2014) 155006.

[468] N. Sakai, J. D. Barrow, Cosmological evolution of black holes in BransDicke gravity, Class. Quantum Grav. 18 (2001) 4717-4724. arXiv:grqc/0102024, doi:10.1088/0264-9381/18/22/301.

[469] D. J. Shaw, J. D. Barrow, Local effects of cosmological variations in physical "constants" and scalar fields. I. Spherically symmetric spacetimes, Phys. Rev. D 73 (2006) 123505.

[470] M. D. Roberts, Imploding scalar fields, J. Math. Phys. 37 (1996) 45574573 .

[471] B. H. Fahim, V. Faraoni, A. Giusti, The Brans-Dicke analogue of the Roberts geometry, Phys. Rev. D 103 (2021) 084004. arXiv:2011.11838, doi:10.1103/PhysRevD.103.084004.

[472] V. Faraoni, Jordan frame no-hair for spherical scalar-tensor black holes, Phys. Rev. D 95 (2017) 124013.

[473] P. Cañate, A no-hair theorem for black holes in $f(R)$ gravity, Class. Quantum Grav. 35 (2018) 025018. doi:10.1088/1361-6382/aa8e2e.

[474] M. Rinaldi, On the equivalence of Jordan and Einstein frames in scaleinvariant gravity, Eur. Phys. J. Plus 133 (2018) 408.

[475] E. Pechlaner, R. Sexl, On quadratic Lagrangians in general relativity, Comm. Math. Phys. 2 (1966) 165-175.

[476] J. Sultana, D. Kazanas, A no-hair theorem for spherically symmetric black holes in $R^{2}$ gravity, Gen. Relativ. Gravit. 50 (2018) 137.

[477] P. J. Ravindranath, Y. Aditya, D. R. K. Reddy, M. V. Subba Rao, Birkhoff's theorem in $f(R)$ theory of gravity, Eur. Phys. J. Plus 133 (2018) 376 .

[478] S. Bhattacharya, Rotating Killing horizons in generic $F(R)$ gravity theories, Gen. Rel. Grav. 48 (2016) 128. arXiv:1602.04306, doi:10.1007/s10714-016-2119-1. 
[479] H.-J. Schmidt, New exact solutions for power-law inflation Friedmann models, Astron. Nach. 311 (1990) 165-168.

[480] J. D. Barrow, T. Clifton, Exact cosmological solutions of scaleinvariant gravity theories, Class. Quantum Grav. 23 (2005) L1.

[481] T. Clifton, Spherically Symmetric Solutions to Fourth-Order Theories of Gravity, Class. Quantum Grav. 23 (2006) 7445. arXiv:grqc/0607096, doi:10.1088/0264-9381/23/24/015.

[482] S. Carloni, P. K. S. Dunsby, S. Capozziello, A. Troisi, Cosmological dynamics of $R^{n}$ gravity, Class. Quantum Grav. 22 (2005) 4839.

[483] J. A. Leach, S. Carloni, P. K. S. Dunsby, Shear dynamics in Bianchi I cosmologies with $R^{n}$-gravity, Class. Quantum Grav. 23 (2006) 49154937. arXiv:gr-qc/0603012, doi:10.1088/0264-9381/23/15/011.

[484] T. Clifton, J. D. Barrow, The power of general relativity, Phys. Rev. D 72 (2005) 103005.

[485] K. C. Chan, J. H. Horne, R. B. Mann, Charged dilaton black holes with unusual asymptotics, Nucl. Phys. B 447 (1995) 441-464. arXiv:grqc/9502042, doi:10.1016/0550-3213(95)00205-7.

[486] G. Cognola, O. Gorbunova, L. Sebastiani, S. Zerbini, Energy issue for a class of modified higher order gravity black hole solutions, Phys. Rev. D 84 (2011) 023515.

[487] A. F. Zakharov, A. A. Nucita, F. De Paolis, G. Ingrosso, Solar system constraints on $R^{n}$ gravity, Phys. Rev. D 74 (2006) 107101.

[488] V. Faraoni, Matter instability in modified gravity, Phys. Rev. D 74 (2006) 104017.

[489] V. Faraoni, Clifton's spherical solution in $f(R)$ vacuum harbours a naked singularity, Class Quantum Grav 26 (2009) 195013.

[490] S. Capozziello, V. Faraoni, Beyond Einstein Gravity: A Survey of Gravitational Theories For Cosmology and Astrophysics, Springer, New York, 2010.

[491] S. Capozziello, M. De Laurentis, Extended Theories of Gravity, Phys. Rept. 509 (2011) 167-321. arXiv:1108.6266, doi:10.1016/j.physrep.2011.09.003. 
[492] B. Whitt, Fourth-order gravity as general relativity plus matter, Phys. Lett. B 145 (1984) 176-178.

[493] S. Mignemi, D. L. Wiltshire, Black holes in higher-derivative gravity theories, Phys. Rev. D 46 (1992) 1475.

[494] K. A. Bronnikov, M. S. Chernakova, Conformal continuations in gravitation theory with Lagrangian $F(R)$, Russ. Phys. J. 48 (2005) 940-946. doi:10.1007/s11182-006-0008-x.

[495] K. A. Bronnikov, M. S. Chernakova, $f(R)$ theory of gravity and conformal continuations (2005). arXiv:gr-qc/0503025.

[496] K. A. Bronnikov, M. S. Chernakova, Charged black holes and unusual wormholes in scalar-tensor gravity (2007). arXiv:gr-qc/0703107.

[497] T. Multamäki, I. Vilja, Spherically symmetric solutions of modified field equations in $f(R)$ theories of gravity, Phys. Rev. D 74 (2006) 064022 .

[498] T. Multamäki, I. Vilja, Static spherically symmetric perfect fluid solutions in $f(R)$ theories of gravity, Phys. Rev. D 76 (2007) 064021.

[499] T. Multamäki, I. Vilja, Constraining Newtonian stellar configurations in $f(R)$ theories of gravity, Phys. Lett. B 659 (2008) 843-846.

[500] A. J. Bustelo, D. E. Barraco, Equilibrium hydrostatic equation and Newtonian limit of the singular $f(R)$ gravity, Class. Quantum Grav. 24 (2007) 2333-2342. arXiv:gr-qc/0611149, doi:10.1088/0264$9381 / 24 / 9 / 011$.

[501] S. Capozziello, M. De Laurentis, M. Francaviglia, Stochastic Background of Gravitational Waves as a Benchmark for Extended Theories of Gravity (2008). arXiv:0812.0168.

[502] A. M. Nzioki, S. Carloni, R. Goswami, P. K. S. Dunsby, New framework for studying spherically symmetric static solutions in $f(R)$ gravity, Phys. Rev. D 81 (2010) 084028.

[503] L. Sebastiani, S. Zerbini, Static spherically symmetric solutions in $f(R)$ gravity, Eur. Phys. J. C 71 (2011) 1591.

[504] R. Myrzakulov, L. Sebastiani, S. Zerbini, Some aspects of generalized modified gravity models, Int. J. Mod. Phys. D 22 (2013) 1330017. 
[505] C. Gao, Y.-G. Shen, Exact solutions in $f(R)$ theory of gravity, Gen. Relativ. Gravit. 48 (2016) 131.

[506] B. Holdom, J. Ren, Not quite a black hole, Phys. Rev. D 95 (2017) 084034 .

[507] M. Calzà, M. Rinaldi, L. Sebastiani, A special class of solutions in $F(R)$-gravity, Eur. Phys. J. C 78 (2018) 178. arXiv:1802.00329, doi:10.1140/epjc/s10052-018-5681-8.

[508] E. Elizalde, G. G. L. Nashed, S. Nojiri, S. D. Odintsov, Spherically symmetric black holes with electric and magnetic charge in extended gravity: physical properties, causal structure, and stability analysis in Einstein's and Jordan's frames, Eur. Phys. J. C 80 (2020) 109. arXiv:2001.11357, doi:10.1140/epjc/s10052-020-7686-3.

[509] N. Deruelle, M. Sasaki, Y. Sendouda, Junction conditions in $f(R)$ theories of gravity, Prog. Theor. Phys. 119 (2008) 237-251.

[510] J. M. M. Senovilla, Junction conditions for $f(R)$ gravity and their consequences, Phys. Rev. D 88 (2013) 064015.

[511] T. Clifton, Embedding Non-Linear Structures in $f(R)$ Cosmologies, in: 14th Marcel Grossmann Meeting on Recent Developments in Theoretical and Experimental General Relativity, Astrophysics, and Relativistic Field Theories, 2015. arXiv:1509.06678.

[512] B. Reina, J. M. M. Senovilla, R. Vera, Junction conditions in quadratic gravity: thin shells and double layers, Class. Quantum Grav. 33 (2016) 105008.

[513] S. Chakrabarti, R. Goswami, S. Maharaj, N. Banerjee, Conformally flat collapsing stars in $f(R)$ gravity, Gen. Rel. Grav. 50 (2018) 148. arXiv:1808.06545, doi:10.1007/s10714-018-2472-3.

[514] D. Lovelock, The Einstein tensor and its generalizations, J. Math. Phys. 12 (1971) 498-501. doi:10.1063/1.1665613.

[515] D. Lovelock, The four-dimensionality of space and the Einstein tensor, J. Math. Phys. 13 (1972) 874-876. doi:10.1063/1.1666069.

[516] M. Ostrogradsky, Mémoires sur les équations différentielles, relatives au problème des isopérimètres, Mem. Acad. St. Petersbourg 6 (4) (1850) 385-517. 
[517] R. P. Woodard, Ostrogradsky's theorem on Hamiltonian instability, Scholarpedia 10 (8) (2015) 32243. arXiv:1506.02210, doi:10.4249/scholarpedia.32243.

[518] A. Nicolis, R. Rattazzi, E. Trincherini, The Galileon as a local modification of gravity, Phys. Rev. D 79 (2009) 064036. arXiv:0811.2197, doi:10.1103/PhysRevD.79.064036.

[519] G. R. Dvali, G. Gabadadze, M. Porrati, 4-D gravity on a brane in 5-D Minkowski space, Phys. Lett. B 485 (2000) 208-214. arXiv:hepth/0005016, doi:10.1016/S0370-2693(00)00669-9.

[520] C. Deffayet, G. Esposito-Farèse, A. Vikman, Covariant Galileon, Phys. Rev. D 79 (2009) 084003. arXiv:0901.1314, doi:10.1103/PhysRevD.79.084003.

[521] T. Kobayashi, M. Yamaguchi, J. Yokoyama, Generalized Ginflation: Inflation with the most general second-order field equations, Prog. Theor. Phys. 126 (2011) 511-529. arXiv:1105.5723, doi:10.1143/PTP.126.511.

[522] C. Deffayet, X. Gao, D. Steer, G. Zahariade, From k-essence to generalised Galileons, Phys. Rev. D 84 (2011) 064039. arXiv:1103.3260, doi:10.1103/PhysRevD.84.064039.

[523] J. Khoury, A. Weltman, Chameleon fields: Awaiting surprises for tests of gravity in space, Phys. Rev. Lett. 93 (2004) 171104. arXiv:astroph/0309300, doi:10.1103/PhysRevLett.93.171104.

[524] J. Khoury, A. Weltman, Chameleon cosmology, Phys. Rev. D 69 (2004) 044026. arXiv:astro-ph/0309411, doi:10.1103/PhysRevD.69.044026.

[525] A. I. Vainshtein, To the problem of nonvanishing gravitation mass, Phys. Lett. B 39 (1972) 393-394. doi:10.1016/0370-2693(72)90147-5.

[526] E. Babichev, C. Deffayet, An introduction to the Vainshtein mechanism, Class. Quant. Grav. 30 (2013) 184001. arXiv:1304.7240, doi:10.1088/0264-9381/30/18/184001.

[527] T. Kobayashi, Horndeski theory and beyond: A review, Rept. Prog. Phys. 82 (8) (2019) 086901. arXiv:1901.07183, doi:10.1088/1361$6633 / \mathrm{ab} 2429$. 
[528] B. P. Abbott, et al., GW170817: Observation of Gravitational Waves from a Binary Neutron Star Inspiral, Phys. Rev. Lett. 119 (2017) 161101. arXiv:1710.05832, doi:10.1103/PhysRevLett.119.161101.

[529] B. P. Abbott, et al., Gravitational Waves and Gamma-rays from a Binary Neutron Star Merger: GW170817 and GRB 170817A, Astrophys. J. Lett. 848 (2017) L13. arXiv:1710.05834, doi:10.3847/20418213/aa920c.

[530] D. Bettoni, J. M. Ezquiaga, K. Hinterbichler, M. Zumalacárregui, Speed of Gravitational Waves and the Fate of Scalar-Tensor Gravity, Phys. Rev. D 95 (8) (2017) 084029. arXiv:1608.01982, doi:10.1103/PhysRevD.95.084029.

[531] M. A. Luty, M. Porrati, R. Rattazzi, Strong interactions and stability in the DGP model, JHEP 09 (2003) 029. arXiv:hep-th/0303116, doi:10.1088/1126-6708/2003/09/029.

[532] C. de Rham, G. Gabadadze, L. Heisenberg, D. Pirtskhalava, Nonrenormalization and naturalness in a class of scalar-tensor theories, Phys. Rev. D 87 (8) (2013) 085017. arXiv:1212.4128, doi:10.1103/PhysRevD.87.085017.

[533] N. Brouzakis, A. Codello, N. Tetradis, O. Zanusso, Quantum corrections in Galileon theories, Phys. Rev. D 89 (12) (2014) 125017. arXiv:1310.0187, doi:10.1103/PhysRevD.89.125017.

[534] D. Pirtskhalava, L. Santoni, E. Trincherini, F. Vernizzi, Weakly Broken Galileon Symmetry, JCAP 09 (2015) 007. arXiv:1505.00007, doi:10.1088/1475-7516/2015/09/007.

[535] L. Santoni, E. Trincherini, L. G. Trombetta, Behind Horndeski: structurally robust higher derivative EFTs, JHEP 08 (2018) 118. arXiv:1806.10073, doi:10.1007/JHEP08(2018)118.

[536] L. Heisenberg, J. Noller, J. Zosso, Horndeski under the quantum loupe, JCAP 10 (2020) 010. arXiv:2004.11655, doi:10.1088/1475$7516 / 2020 / 10 / 010$.

[537] L. Hui, A. Nicolis, No-Hair Theorem for the Galileon, Phys. Rev. Lett. 110 (2013) 241104. arXiv:1202.1296, doi:10.1103/PhysRevLett.110.241104. 
[538] H. O. Silva, A. Maselli, M. Minamitsuji, E. Berti, Compact objects in Horndeski gravity, Int. J. Mod. Phys. D 25 (2016) 1641006. doi:doi:10.1142/S0218271816410066.

[539] E. Babichev, C. Charmousis, Dressing a black hole with a time-dependent Galileon, JHEP 08 (2014) 106. arXiv:1312.3204, doi:10.1007/JHEP08(2014)106.

[540] C. Charmousis, B. Gouteraux, E. Kiritsis, Higher-derivative scalarvector-tensor theories: black holes, Galileons, singularity cloaking and holography, J. High Energy Phys. 09 (2012) 011. arXiv:1206.1499, doi:10.1007/JHEP09(2012)011.

[541] T. P. Sotiriou, S.-Y. Zhou, Black hole hair in generalized scalartensor gravity: An explicit example, Phys. Rev. D 90 (2014) 124063. arXiv:1408.1698, doi:10.1103/PhysRevD.90.124063.

[542] B. A. Campbell, N. Kaloper, K. A. Olive, Classical hair for KerrNewman black holes in stringy gravity, Phys. Lett. B 285 (1992) 199205. doi:10.1016/0370-2693(92)91452-F.

[543] P. Kanti, N. E. Mavromatos, J. Rizos, K. Tamvakis, E. Winstanley, Dilatonic black holes in higher curvature string gravity, Phys. Rev. D 54 (1996) 5049-5058. arXiv:hep-th/9511071, doi:10.1103/PhysRevD.54.5049.

[544] C. Charmousis, E. J. Copeland, A. Padilla, P. M. Saffin, General second order scalar-tensor theory, self tuning, and the Fab Four, Phys. Rev. Lett. 108 (2012) 051101. arXiv:1106.2000, doi:10.1103/PhysRevLett.108.051101.

[545] M. Rinaldi, Black holes with non-minimal derivative coupling, Phys. Rev. D 86 (2012) 084048. arXiv:1208.0103, doi:10.1103/PhysRevD.86.084048.

[546] A. Anabalón, A. Cisterna, J. Oliva, Asymptotically locally AdS and flat black holes in Horndeski theory, Phys. Rev. D 89 (2014) 084050. arXiv:1312.3597, doi:10.1103/PhysRevD.89.084050.

[547] M. Minamitsuji, Solutions in the scalar-tensor theory with nonminimal derivative coupling, Phys. Rev. D 89 (2014) 064017. arXiv:1312.3759, doi:10.1103/PhysRevD.89.064017. 
[548] D. Langlois, K. Noui, Degenerate higher derivative theories beyond Horndeski: Evading the Ostrogradski instability, JCAP 02 (2016) 034. arXiv:1510.06930, doi:10.1088/1475-7516/2016/02/034.

[549] D. Langlois, K. Noui, Hamiltonian analysis of higher derivative scalar-tensor theories, JCAP 07 (2016) 016. arXiv:1512.06820, doi:10.1088/1475-7516/2016/07/016.

[550] J. Ben Achour, D. Langlois, K. Noui, Degenerate higher order scalar-tensor theories beyond Horndeski and disformal transformations, Phys. Rev. D 93 (12) (2016) 124005. arXiv:1602.08398, doi:10.1103/PhysRevD.93.124005.

[551] M. Crisostomi, K. Koyama, G. Tasinato, Extended Scalar-Tensor Theories of Gravity, JCAP 04 (2016) 044. arXiv:1602.03119, doi:10.1088/1475-7516/2016/04/044.

[552] H. Motohashi, K. Noui, T. Suyama, M. Yamaguchi, D. Langlois, Healthy degenerate theories with higher derivatives, JCAP 07 (2016) 033. arXiv:1603.09355, doi:10.1088/1475-7516/2016/07/033.

[553] J. Ben Achour, M. Crisostomi, K. Koyama, D. Langlois, K. Noui, G. Tasinato, Degenerate higher order scalar-tensor theories beyond Horndeski up to cubic order, JHEP 12 (2016) 100. arXiv:1608.08135, doi:10.1007/JHEP12(2016)100.

[554] M. Crisostomi, R. Klein, D. Roest, Higher Derivative Field Theories: Degeneracy Conditions and Classes, JHEP 06 (2017) 124. arXiv:1703.01623, doi:10.1007/JHEP06(2017)124.

[555] D. Langlois, R. Saito, D. Yamauchi, K. Noui, Scalar-tensor theories and modified gravity in the wake of GW170817, Phys. Rev. D 97 (6) (2018) 061501. arXiv:1711.07403, doi:10.1103/PhysRevD.97.061501.

[556] P. Creminelli, M. Lewandowski, G. Tambalo, F. Vernizzi, Gravitational Wave Decay into Dark Energy, JCAP 12 (2018) 025. arXiv:1809.03484, doi:10.1088/1475-7516/2018/12/025.

[557] E. Babichev, C. Charmousis, G. Esposito-Farèse, A. Lehébel, Stability of Black Holes and the Speed of Gravitational Waves within Self-Tuning Cosmological Models, Phys. Rev. Lett. 120 (24) (2018) 241101. arXiv:1712.04398, doi:10.1103/PhysRevLett.120.241101. 
[558] E. Babichev, C. Charmousis, G. Esposito-Farèse, A. Lehébel, Hamiltonian unboundedness vs stability with an application to Horndeski theory, Phys. Rev. D 98 (10) (2018) 104050. arXiv:1803.11444, doi:10.1103/PhysRevD.98.104050.

[559] K. Takahashi, H. Motohashi, M. Minamitsuji, Linear stability analysis of hairy black holes in quadratic degenerate higher-order scalartensor theories: Odd-parity perturbations, Phys. Rev. D 100 (2) (2019) 024041. arXiv:1904.03554, doi:10.1103/PhysRevD.100.024041.

[560] C. de Rham, J. Zhang, Perturbations of stealth black holes in degenerate higher-order scalar-tensor theories, Phys. Rev. D 100 (12) (2019) 124023. arXiv:1907.00699, doi:10.1103/PhysRevD.100.124023.

[561] D. Langlois, Dark energy and modified gravity in degenerate higher-order scalar-tensor (DHOST) theories: A review, Int. J. Mod. Phys. D 28 (05) (2019) 1942006. arXiv:1811.06271, doi:10.1142/S0218271819420069.

[562] C. Charmousis, M. Crisostomi, R. Gregory, N. Stergioulas, Rotating Black Holes in Higher Order Gravity, Phys. Rev. D 100 (8) (2019) 084020. arXiv:1903.05519, doi:10.1103/PhysRevD.100.084020.

[563] M. Minamitsuji, Disformal transformation of stationary and axisymmetric solutions in modified gravity, Phys. Rev. D 102 (12) (2020) 124017. arXiv:2012.13526, doi:10.1103/PhysRevD.102.124017.

[564] F. Long, S. Chen, M. Wang, J. Jing, Shadow of a disformal Kerr black hole in quadratic degenerate higher-order scalar-tensor theories, Eur. Phys. J. C 80 (12) (2020) 1180. arXiv:2009.07508, doi:10.1140/epjc/s10052-020-08744-8.

[565] J. Ben Achour, H. Liu, H. Motohashi, S. Mukohyama, K. Noui, On rotating black holes in DHOST theories, JCAP 11 (2020) 001. arXiv:2006.07245, doi:10.1088/1475-7516/2020/11/001.

[566] T. Anson, E. Babichev, C. Charmousis, M. Hassaine, Disforming the Kerr metric, JHEP 01 (2021) 018. arXiv:2006.06461, doi:10.1007/JHEP01(2021)018.

[567] E. Babichev, K. Koyama, D. Langlois, R. Saito, J. Sakstein, Relativistic Stars in Beyond Horndeski Theories, Class. Quant. Grav. 33 (23) (2016) 235014. arXiv:1606.06627, doi:10.1088/0264$9381 / 33 / 23 / 235014$. 
[568] J. Sakstein, E. Babichev, K. Koyama, D. Langlois, R. Saito, Towards Strong Field Tests of Beyond Horndeski Gravity Theories, Phys. Rev. D 95 (6) (2017) 064013. arXiv:1612.04263, doi:10.1103/PhysRevD.95.064013.

[569] T. Kobayashi, T. Hiramatsu, Relativistic stars in degenerate higherorder scalar-tensor theories after GW170817, Phys. Rev. D 97 (10) (2018) 104012. arXiv:1803.10510, doi:10.1103/PhysRevD.97.104012.

[570] E. Babichev, A. Lehébel, The sound of DHOST, JCAP 12 (2018) 027. arXiv:1810.09997, doi:10.1088/1475-7516/2018/12/027.

[571] A. Cisterna, T. Delsate, M. Rinaldi, Neutron stars in general second order scalar-tensor theory: The case of nonminimal derivative coupling, Phys. Rev. D 92 (4) (2015) 044050. arXiv:1504.05189, doi:10.1103/PhysRevD.92.044050.

[572] A. Cisterna, T. Delsate, L. Ducobu, M. Rinaldi, Slowly rotating neutron stars in the nonminimal derivative coupling sector of Horndeski gravity, Phys. Rev. D 93 (8) (2016) 084046. arXiv:1602.06939, doi:10.1103/PhysRevD.93.084046.

[573] A. Lehébel, E. Babichev, C. Charmousis, A no-hair theorem for stars in Horndeski theories, JCAP 07 (2017) 037. arXiv:1706.04989, doi:10.1088/1475-7516/2017/07/037.

[574] E. Babichev, C. Charmousis, A. Lehébel, Asymptotically flat black holes in Horndeski theory and beyond, JCAP 04 (2017) 027. arXiv:1702.01938, doi:10.1088/1475-7516/2017/04/027.

[575] E. Babichev, G. Esposito-Farèse, Cosmological self-tuning and local solutions in generalized Horndeski theories, Phys. Rev. D 95 (2) (2017) 024020. arXiv:1609.09798, doi:10.1103/PhysRevD.95.024020.

[576] H. Motohashi, M. Minamitsuji, General Relativity solutions in modified gravity, Phys. Lett. B 781 (2018) 728-734. arXiv:1804.01731, doi:10.1016/j.physletb.2018.04.041.

[577] M. Minamitsuji, H. Motohashi, Stealth Schwarzschild solution in shift symmetry breaking theories, Phys. Rev. D 98 (8) (2018) 084027. arXiv:1809.06611, doi:10.1103/PhysRevD.98.084027. 
[578] E. Babichev, G. Esposito-Farèse, Time-Dependent Spherically Symmetric Covariant Galileons, Phys. Rev. D 87 (2013) 044032. arXiv:1212.1394, doi:10.1103/PhysRevD.87.044032.

[579] C. Charmousis, T. Kolyvaris, E. Papantonopoulos, M. Tsoukalas, Black Holes in Bi-scalar Extensions of Horndeski Theories, JHEP 07 (2014) 085. arXiv:1404.1024, doi:10.1007/JHEP07(2014)085.

[580] T. Kobayashi, N. Tanahashi, Exact black hole solutions in shift symmetric scalar-tensor theories, PTEP 2014 (2014) $073 \mathrm{E} 02$. arXiv:1403.4364, doi:10.1093/ptep/ptu096.

[581] J. Ben Achour, H. Liu, Hairy Schwarzschild-(A)dS black hole solutions in degenerate higher order scalar-tensor theories beyond shift symmetry, Phys. Rev. D 99 (6) (2019) 064042. arXiv:1811.05369, doi:10.1103/PhysRevD.99.064042.

[582] H. Motohashi, M. Minamitsuji, Exact black hole solutions in shift-symmetric quadratic degenerate higher-order scalar-tensor theories, Phys. Rev. D 99 (6) (2019) 064040. arXiv:1901.04658, doi:10.1103/PhysRevD.99.064040.

[583] M. Minamitsuji, J. Edholm, Black hole solutions in shift-symmetric degenerate higher-order scalar-tensor theories, Phys. Rev. D 100 (4) (2019) 044053. arXiv:1907.02072, doi:10.1103/PhysRevD.100.044053.

[584] H. Motohashi, S. Mukohyama, Weakly-coupled stealth solution in scordatura degenerate theory, JCAP 01 (2020) 030. arXiv:1912.00378, doi:10.1088/1475-7516/2020/01/030.

[585] S. Mukohyama, Black holes in the ghost condensate, Phys. Rev. D 71 (2005) 104019. arXiv:hep-th/0502189, doi:10.1103/PhysRevD.71.104019.

[586] S. Mukohyama, Ghost condensate and generalized second law, JHEP 09 (2009) 070. arXiv:0901.3595, doi:10.1088/1126-6708/2009/09/070.

[587] N. Arkani-Hamed, H.-C. Cheng, M. A. Luty, S. Mukohyama, Ghost condensation and a consistent infrared modification of gravity, JHEP 05 (2004) 074. arXiv:hep-th/0312099, doi:10.1088/1126$6708 / 2004 / 05 / 074$. 
[588] N. Arkani-Hamed, P. Creminelli, S. Mukohyama, M. Zaldarriaga, Ghost inflation, JCAP 04 (2004) 001. arXiv:hep-th/0312100, doi:10.1088/1475-7516/2004/04/001.

[589] J. Ben Achour, H. Liu, S. Mukohyama, Hairy black holes in DHOST theories: Exploring disformal transformation as a solution-generating method, JCAP 02 (2020) 023. arXiv:1910.11017, doi:10.1088/1475$7516 / 2020 / 02 / 023$.

[590] M. Minamitsuji, J. Edholm, Black holes with a nonconstant kinetic term in degenerate higher-order scalar tensor theories, Phys. Rev. D 101 (4) (2020) 044034. arXiv:1912.01744, doi:10.1103/PhysRevD.101.044034.

[591] A. L. Alinea, On the Disformal Transformation of the Einstein-Hilbert Action (10 2020). arXiv:2010.00956.

[592] M. A. Gorji, H. Motohashi, S. Mukohyama, Stealth dark energy in scordatura DHOST theory, JCAP 03 (2021) 081. arXiv:2009.11606, doi:10.1088/1475-7516/2021/03/081.

[593] J. Khoury, M. Trodden, S. S. C. Wong, Existence and instability of hairy black holes in shift-symmetric Horndeski theories, JCAP 11 (2020) 044. arXiv:2007.01320, doi:10.1088/1475-7516/2020/11/044.

[594] M. Khodadi, A. Allahyari, S. Vagnozzi, D. F. Mota, Black holes with scalar hair in light of the Event Horizon Telescope, JCAP 09 (2020) 026. arXiv:2005.05992, doi:10.1088/1475-7516/2020/09/026.

[595] C. Deffayet, S. Garcia-Saenz, Degeneracy, matter coupling, and disformal transformations in scalar-tensor theories, Phys. Rev. D 102 (6) (2020) 064037. arXiv:2004.11619, doi:10.1103/PhysRevD.102.064037.

[596] K. Takahashi, H. Motohashi, General Relativity solutions with stealth scalar hair in quadratic higher-order scalar-tensor theories, JCAP 06 (2020) 034. arXiv:2004.03883, doi:10.1088/1475-7516/2020/06/034.

[597] L. Bel, H. S. Zia, Regular reduction of relativistic theories of gravitation with a quadratic Lagrangian, Phys. Rev. D 32 (1985) 3128.

[598] J. Z. Simon, Higher-derivative Lagrangians, nonlocality, problems, and solutions, Phys. Rev. D 41 (1990) 3720. 
[599] S. DeDeo, D. Psaltis, Stable, accelerating universes in modified-gravity theories, Phys. Rev. D 78 (2008) 064013.

[600] P. Mészáros, D. B. Fox, C. Hanna, K. Murase, Multi-messenger astrophysics, Nature Rev. Phys. 1 (2019) 585-599. 